\title{
The Electric and Magnetic Field Instrument Suite and Integrated Science (EMFISIS) on RBSP
}

\author{
C.A. Kletzing • W.S. Kurth • M. Acuna • R.J. MacDowall • R.B. Torbert • \\ T. Averkamp · D. Bodet $\cdot$ S.R. Bounds $\cdot$ M. Chutter $\cdot$ J. Connerney $\cdot$ D. Crawford • \\ J.S. Dolan · R. Dvorsky • G.B. Hospodarsky • J. Howard • V. Jordanova • \\ R.A. Johnson - D.L. Kirchner • B. Mokrzycki - G. Needell • J. Odom • D. Mark • \\ R. Pfaff Jr. • J.R. Phillips • C.W. Piker • S.L. Remington • D. Rowland • O. Santolik • \\ R. Schnurr · D. Sheppard · C.W. Smith $\cdot$ R.M. Thorne $\cdot$ J. Tyler
}

Received: 30 August 2012 / Accepted: 9 May 2013 / Published online: 1 June 2013

(C) The Author(s) 2013. This article is published with open access at Springerlink.com

\begin{abstract}
The Electric and Magnetic Field Instrument and Integrated Science (EMFISIS) investigation on the NASA Radiation Belt Storm Probes (now named the Van Allen Probes) mission provides key wave and very low frequency magnetic field measurements to understand radiation belt acceleration, loss, and transport. The key science objectives and the contribution that EMFISIS makes to providing measurements as well as theory and modeling are described. The key components of the instruments suite, both electronics and sensors,
\end{abstract}

C.A. Kletzing $(\varangle) \cdot$ W.S. Kurth · T. Averkamp · S.R. Bounds · D. Crawford · J.S. Dolan · R. Dvorsky ·

G.B. Hospodarsky · J. Howard · R.A. Johnson - D.L. Kirchner · B. Mokrzycki · J.R. Phillips ·

C.W. Piker · S.L. Remington

Department of Physics \& Astronomy, University of Iowa, Iowa City, IA, USA

e-mail: craig-kletzing@uiowa.edu

M. Acuna · R.J. MacDowall · J. Connerney · J. Odom · R. Schnurr · D. Sheppard

Solar System Exploration Division, Goddard Space Flight Center, Greenbelt, MD, USA

R.B. Torbert · D. Bodet · M. Chutter · G. Needell $\cdot$ C.W. Smith · J. Tyler

Physics Department and Space Science Center, University of New Hampshire, Durham, NH, USA

R.M. Thorne

Atmospheric and Oceanic Sciences, University of California, Los Angeles, CA, USA

V. Jordanova

Space Science and Applications, Los Alamos National Laboratory, Los Alamos, NM, USA

O. Santolik

Department of Space Physics, Institute of Atmospheric Physics, Prague, Czech Republic

O. Santolik

Faculty of Mathematics and Physics, Charles University, Prague, Czech Republic

D. Mark

Bison Aerospace Inc., Newcastle, WY, USA

R. Pfaff Jr. · D. Rowland

Heliophysics Science Division, Goddard Space Flight Center, Greenbelt, MD, USA 
including key functional parameters, calibration, and performance, demonstrate that EMFISIS provides the needed measurements for the science of the RBSP mission. The EMFISIS operational modes and data products, along with online availability and data tools provide the radiation belt science community with one the most complete sets of data ever collected.

Keywords Radiation belt physics - Wave measurements · Magnetometer measurements · Space flight instruments - RBSP - Radiation belt storm probes - Van Allen probes · Whistler waves $\cdot$ Geomagnetic storms $\cdot$ Space weather

\section{Introduction}

Radiation belt electrons are distributed in two distinct zones: the inner zone that is relatively stable and the outer zone that is very dynamic. The flux of energetic electrons in the Earth's outer radiation belt can vary by several orders of magnitude over time scales less than a day in response to changes in properties of the solar wind instigated by solar activity. Variability in the radiation belts is due to an imbalance between the dominant source and loss processes that are caused by a violation of one or more of the adiabatic invariants. For radiation belt electrons, non-adiabatic behavior is primarily associated with energy and momentum transfer during interactions with various magnetospheric waves (Thorne 2010). The most dramatic variations in the outer belt occur during magnetic storms, when the enhancement in the ring current causes a global decrease in the ambient magnetic field (e.g., Tsyganenko and Stern 1996). Reduced magnetic field affects the adiabatic drifts of radiation belt particles and causes an outward motion of radiation belt electrons with an accompanying loss of energy, which in turn causes an adiabatic decrease in radiation belt flux (e.g., Kim and Chan 1997) in addition to loss by scattering into the atmosphere (Millan and Thorne 2007) and transport to the magnetopause (Ukhorskiy et al. 2006). To differentiate between adiabatic and non-adiabatic changes in the radiation belts a simultaneous study of the radiation belts and ring current dynamics is needed (Jordanova 2012).

There are three adiabatic invariants (Roederer 1970) associated with the three basic periodic motions: gyromotion, the bounce motion in the Earth's magnetic mirror field, and the azimuthal drift due to magnetic gradients. Each invariant can be violated when the system is subject to fluctuations on timescales comparable to or shorter than the associated periodic motion (Schulz and Lanzerotti 1974). ULF waves, with periods comparable to tens of minutes, cause a violation of the third invariant, resulting in radial diffusion. Since the power in ULF waves is considerably enhanced during magnetic storms (Mathie and Mann 2000), radial diffusion is a potentially important mechanism for energetic electron acceleration (Rostoker et al. 1998; Elkington et al. 1999; Hudson et al. 2001; O'Brien et al. 2001; Shprits and Thorne 2004) or loss (Shprits et al. 2006; Jordanova et al. 2008; Loto'aniu et al. 2010) during storm conditions, dependent on the radial gradient in phase space density. Higher frequency ELF and VLF waves cause violation of the first two invariants and lead to pitch angle scattering loss to the atmosphere (Thorne and Kennel 1971; Lyons et al. 1972; Abel and Thorne 1998a, 1998b) or local stochastic energy diffusion (Horne and Thorne 1998; Summers et al. 1998; Horne et al. 2005; Miyoshi et al. 2003).

During storm conditions, the power spectral density of ELF and VLF waves is also strongly enhanced (Meredith et al. 2003a; Li et al. 2009b). Consequently, during disturbed conditions, all three adiabatic invariants can be violated simultaneously, and multidimensional diffusion models (Jordanova and Miyoshi 2005; Jordanova et al. 2008; Fok et al. 2008; Tao et al. 2008, 2009; Varotsou et al. 2008; Albert et al. 2009; Shprits et al. 2009; 


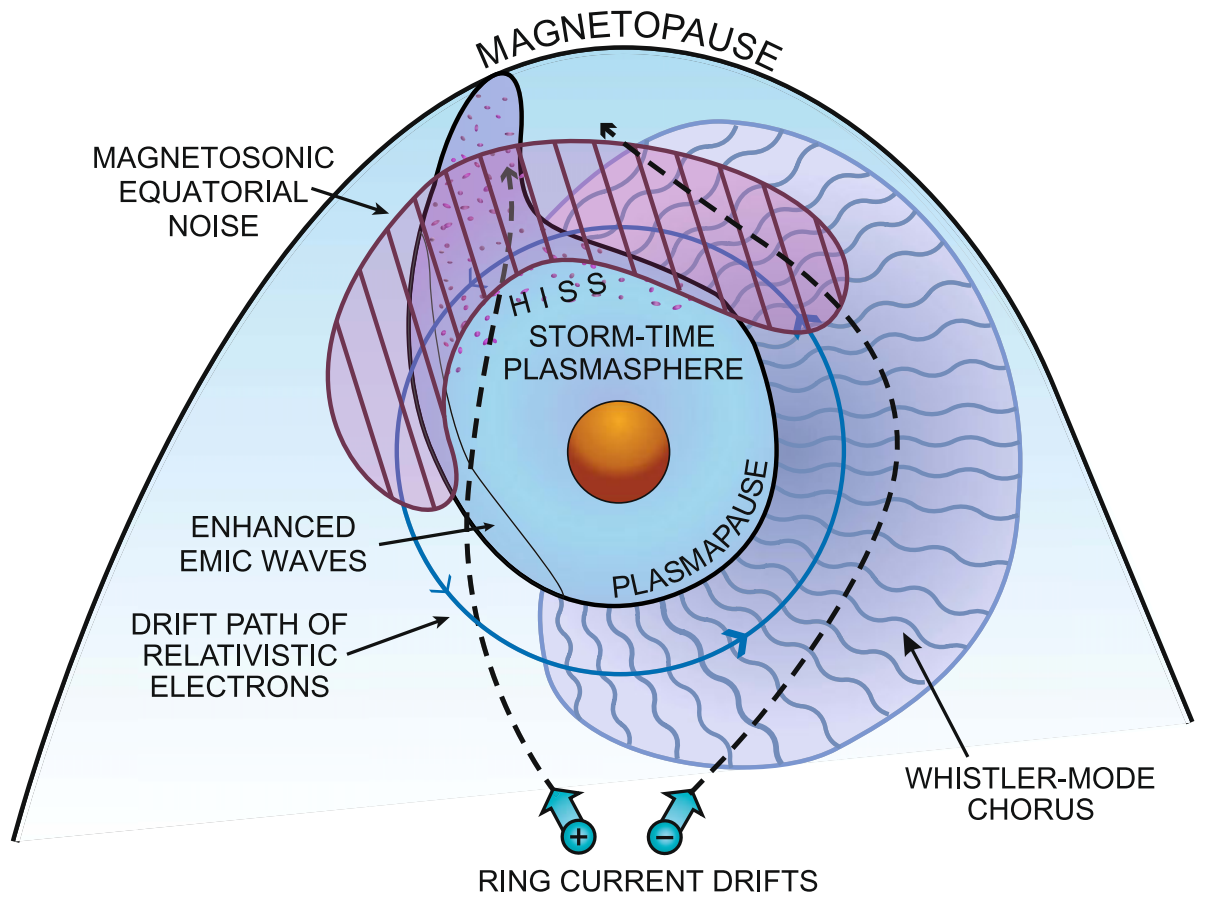

Fig. 1 A schematic diagram of the equatorial magnetosphere illustrating the spatial regions for wave-particle interactions between relativistic electrons and important plasma waves. Whistler-mode chorus can induce microburst precipitation (Thorne et al. 2005) and local stochastic acceleration (Summers et al. 1998; Horne et al. 2005) along a broad portion of the electron drift path between midnight and noon. Equatorial magnetosonic waves contribute to local electron acceleration. Strong pitch angle scattering by EMIC waves along the duskside plasmapause can cause intense but localized precipitation in the dusk sector Jordanova et al. (2008). Electrons are also subject to weak diffusion scattering on the dayside during resonance with plasmaspheric hiss. Relativistic electron drift times are typically less than 10 minutes and the average rate of precipitation loss or stochastic acceleration must be averaged over both the bounce and drift motions in this highly variable environment

Subbotin and Shprits 2009; Tu et al. 2009) are required to differentiate between the different source and loss processes. Such codes require accurate specification of the rate of radial diffusion $D_{L L}$, pitch angle scattering $D_{\alpha \alpha}$, and energy (or momentum) diffusion $D_{E E}$. Each diffusion coefficient requires a global specification of the power spectral density of all relevant plasma waves. A schematic model for the regions where some of these waves are excited is given in Fig. 1. The excited waves cause particle scattering, which modifies the particle pitch angle distribution and leads to loss in the atmosphere. During resonant scattering, energy can also be transferred from the low energy population (which provides the source of the waves) to the high energy tail population, causing in situ local acceleration (Horne and Thorne 2003). The variability of such scattering during geomagnetically active periods requires precise measurement of the intensity of all important magnetospheric waves. Accurate measurement of the large scale magnetic fields is needed to determine the evolution of ring current as well as the source population and/or the adiabatic effects on energetic electrons. The magnetospheric waves responsible for radiation belt dynamics have recently been reviewed by Thorne (2010). A brief summary of our current understanding of these waves is given below. 


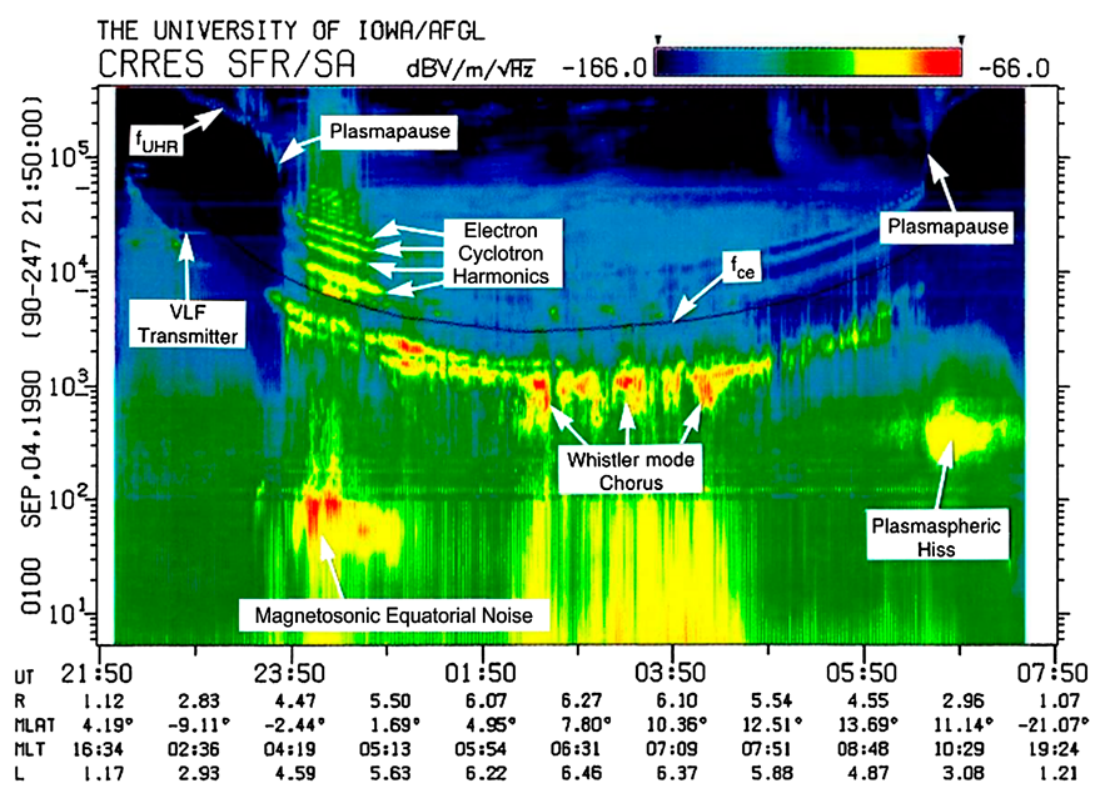

Fig. 2 Spectrogram of waves observed on Combined Release and Radiation Effects Satellite (CRRES), showing various magnetospheric waves which resonate with energetic electrons

Ultra Low Frequency Waves $(2-100 \mathrm{mHz}$ ) are excited at the magnetopause boundary in response to velocity shear (Claudepierre et al. 2008) or solar wind pressure fluctuations (Ukhorskiy et al. 2006; Claudepierre et al. 2009). Hydromagnetic waves may also be excited internally by natural instability of the magnetospheric plasma. These ULF waves cause radial diffusion transport and associated energy change in the trapped particle population. The rate of radial transport is dependent on the power spectral density of the waves and tends to be much faster in the outer magnetosphere.

Chorus Emissions $\left(0.1-0.7 f_{c e}\right)$ are discrete coherent whistler mode waves, which occur in two distinct bands above and below one-half the electron cyclotron frequency $f_{c e}$ as shown in Fig. 2 (Tsurutani and Smith 1974). Chorus is important because it plays a dual role in both the loss and local acceleration of radiation belt electrons (Bortnik and Thorne 2007) and is the dominant scattering process leading to diffuse auroral precipitation ( $\mathrm{Ni}$ et al. 2008; Nishimura et al. 2010; Thorne et al. 2010). Recent statistical analyses of the global distribution of chorus observed on the THEMIS spacecraft indicates that the power spectral density is highly variable and responds to geomagnetic activity Li et al. (2009b). Chorus is enhanced over a broad spatial region (Hayosh et al. 2010) exterior to the plasmapause (see Fig. 1) associated with cyclotron resonant excitation during the convective injection of plasma sheet electrons into the magnetosphere ( $\mathrm{Li}$ et al. 2008, 2009a; Jordanova et al. 2010a). Nightside chorus is strongest inside $L=8$, and is also confined to latitudes below $15^{\circ}$, due to strong Landau damping of oblique waves during their propagation towards higher latitude from the equatorial source region (Bortnik et al. 2007). In contrast, dayside chorus is found over a broad range of latitudes, is most intense in the outer $(L \approx 8)$ magnetosphere, and shows less dependence on geomagnetic activity (Tsurutani and Smith 1977; $\mathrm{Li}$ et al. 2009b). The wave normal distribution of chorus is required to accurately evaluate resonant electron energies and quantify the associated rates of scattering (Shprits and Ni 
2009). Unfortunately, recent satellite observations (Chum et al. 2007; Breneman et al. 2009; Santolik et al. 2009; Haque et al. 2010) indicate a wide range of values for this key property, which adds uncertainty to modeling studies.

Equatorial Magnetosonic Waves $\left(<f_{l h}\right)$ are highly oblique whistler-mode emissions excited within a few degrees of the equatorial plane at frequencies between the proton cyclotron frequency and the lower hybrid (e.g., Santolik et al. 2004). The waves are observed both inside and outside the plasmapause and are excited by a cyclotron resonant instability with a ring distribution of injected ring current ions (Horne et al. 2000; Meredith et al. 2008; Chen et al. 2010b). MS waves also undergo a Landau resonance with radiation belt ( $100 \mathrm{keV}$ to a few $\mathrm{MeV}$ ) electrons, and the spectral properties of intense MS waves observed on Cluster have been used to demonstrate that the timescale for energy diffusion ( $\sim$ day) can be comparable to that due to chorus scattering (Horne et al. 2007). Test particle scattering of electrons in a finite amplitude MS wave have confirmed the rate of Landau resonant scattering (Bortnik and Thorne 2010) and demonstrated additional non-resonant transit time scattering due to the equatorial confinement of MS wave power.

Plasmaspheric Hiss (100 Hz-( 2) $\mathrm{kHz}$ ) is an incoherent whistler-mode emission mostly confined within the dense plasmasphere and within dayside plasmaspheric plumes, which is mainly responsible for the formation of the quiet time electron slot between the inner and outer radiation belt (Lyons and Thorne 1973; Abel and Thorne 1998a). Recent ray trace modeling has shown that hiss originates from a subset of chorus emissions that avoid Landau damping during propagation from the equatorial source region to higher latitude. Such waves also propagate to lower $L$ where they enter and are trapped within the plasmasphere, where the discrete chorus emissions merge together to form incoherent hiss (Bortnik et al. 2008b, 2009a). The unexpected association between hiss and chorus has been confirmed by simultaneous observations on two THEMIS spacecraft (Bortnik et al. 2009b) and differences in the statistical MLT distribution of the two emissions has been explained by 3D ray tracing (Chen et al. 2009b).

Electromagnetic Ion Cyclotron Waves (EMIC, $<1-2 \mathrm{~Hz}$ ) are discrete electromagnetic emissions, which occur in distinct frequency bands separated by multiple ion gyrofrequencies. The EMIC source region is typically confined within $\approx 10$ degrees of the geomagnetic equatorial plane, and the Poynting flux at higher latitude is always directed away from the equator, dispelling the long-standing bouncing wave packet model (Loto'aniu et al. 2005). EMIC waves are enhanced during magnetic storms (Fraser et al. 2010), as anisotropic energetic ring current ions are injected into the inner magnetosphere (Jordanova et al. 2001a). EMIC waves can cause rapid scattering and loss for ring current ions (Jordanova et al. 2001b, 2006) and relativistic electrons above $0.5 \mathrm{MeV}$ (Thorne and Kennel 1971; Lyons and Thorne 1973; Albert 2003; Summers and Thorne 2003; Meredith et al. 2003b). Favored regions for EMIC excitation include the overlap between the ring current and the plasmasphere (Pickett et al. 2010), dayside drainage plumes (Morley et al. 2009), and the outer dayside magnetosphere in association with solar wind pressure fluctuations (Arnoldy et al. 2005; Usanova et al. 2008; McCollough et al. 2009). Theoretical global modeling of EMIC wave excitation has confirmed the plasmapause and plume as favored regions of cyclotron resonant instability (Jordanova et al. 2007; Chen et al. 2010a) and demonstrated that the wave excitation can also be enhanced by density fluctuations within a plume (Chen et al. 2009a). Hybrid codes have recently been used to evaluate the spectral properties and ultimate saturation amplitudes of EMIC waves (Hu and Denton 2009; Omidi et al. 2010). 
Electron Cyclotron Harmonic $\left(E C H, f_{c e}(n+1 / 2)\right)$ waves are electrostatic emissions, which occur in harmonic bands between multiples of the electron cyclotron frequency. These waves are excited by the loss cone instability of injected plasma sheet electrons (e.g., Horne and Thorne 2000). The global distribution of ECH emission intensity and its dependence on geomagnetic activity has been analyzed by Meredith et al. (2009) and shown to be similar to that of chorus. Although ECH emissions contribute to the scattering loss of plasma sheet electrons below a few $\mathrm{keV}$ at larger $L(L>8)(\mathrm{Ni}$ et al. 2011) ECH waves play little role in energetic $(>30 \mathrm{keV})$ radiation belt dynamics.

The Electric and Magnetic Field Instrument Suite and Integrated Science (EMFISIS) investigation provides the key wave and DC (defined as $0-30 \mathrm{~Hz}$ for EMFISIS) magnetic field observations which, together with the EFW electric field measurements and the RBSP particle measurements, will allow us to identify the origin of all plasma waves important for radiation belt physics, as well as the evolution of the storm-time ring current, and to quantify their influences on the variability of trapped radiation belt particles.

On November 9, 2012 after the completion of commissioning of the instruments for RBSP, NASA renamed the Radiation Belt Storm Probes (RBSP) mission the Van Allen Probes mission. In what follows, we use the RBSP acronym to maintain consistency with other Van Allen Probes instrument papers, but future work of EMFISIS investigation will use the new name.

\section{Science Goals and Objectives}

The EMFISIS wave and magnetic field observations will address several key science objectives for the RBSP mission. Specifically, EMFISIS addresses the three overarching Level 1 Science questions:

\subsection{Which Physical Processes Produce Radiation Belt Enhancement Events?}

An essential unanswered question of inner magnetospheric dynamics is how electrons are accelerated to relativistic ( $\mathrm{MeV})$ energies following some magnetic storms. Comprehensive studies at geosynchronous orbit have indicated that acceleration in that region is correlated with enhanced ULF waves (Mathie and Mann 2000; O'Brien et al. 2001; Green and Kivelson 2001). However, the heart of the radiation belts lies well inside geosynchronous orbit, in the region near 3-5 RE. Theoretical calculations suggest that ULF acceleration mechanisms should be substantially reduced in efficiency at lower $L$, compared to geosynchronous orbit (Fälthammar and Walt 1969; Elkington et al. 2003). On the other hand, local acceleration involving VLF waves, particularly lower-band chorus, becomes most efficient in the region just outside the plasmapause, which corresponds to the radial range 3-5 RE for storm conditions (Summers et al. 1998; Meredith et al. 2003b).

Radial Transport and Acceleration by ULF Waves The global distribution and variability of low frequency Pc4 and Pc5 waves can be monitored by ground-based magnetometers and by satellites (Liu et al. 2009), and the observed wave spectral characteristics have been used to evaluate radial diffusion coefficients (Brautigam et al. 2005; Perry et al. 2005; Ukhorskiy et al. 2005; Huang et al. 2010) and employed in dynamic modeling of the outer radiation belt (Loto'aniu et al. 2006; Ukhorskiy et al. 2009; Chu et al. 2010). The properties of magnetospheric ULF waves, excited in response to solar wind variability, have also been obtained from global MHD simulations and used 
to study the dynamic variability of radiation belt electrons (Fei et al. 2006; Kress et al. 2007). Although radial diffusion transport is able to simulate several important features of radiation belt dynamics, it fails to describe the rapid flux variation and the prolonged duration of electron acceleration observed during individual storms (Miyoshi et al. 2001; Subbotin and Shprits 2009). The ability of ULF waves to cause effective radial diffusion depends on the amplitude and the poloidal or toroidal properties of the waves and their modal structure (Perry et al. 2005). The azimuthal, radial, and field-aligned mode structure of ULF waves in space will be determined by multipoint EMFISIS measurements of magnetic fields on the two RBSP spacecraft.

Local Acceleration by Whistler-Mode Waves Persistent peaks in energetic electron phase space density have been identified in the heart of the outer radiation zone ( $L \approx 5$ ) (Green and Kivelson 2004; Chen et al. 2006b, 2007), which support earlier theoretical studies of the importance of local stochastic acceleration (Summers et al. 1998; Horne and Thorne 1998). Potential mechanisms responsible for the local acceleration to relativistic energies during the recovery phase of a storm include cyclotron resonant interactions with VLF chorus in the low-density region just outside the plasmapause (Horne et al. 2005) and Landau resonance with equatorial magnetosonic waves (Horne et al. 2007). The rate of acceleration is strongly dependent on plasma density, specifically, on the ratio between the electron cyclotron frequency and the plasma frequency.

The EMFISIS wave instruments provide measurements of the power spectral density of VLF waves every $6 \mathrm{~s}$, and a full 3D spectral matrix with the same cadence along with a selection of burst modes which include full waveforms from all three axes of the electric and magnetic field sensors. This information, together with our measurements of plasma density, are critical for understanding the effectiveness of local acceleration. Our observations, in conjunction with RBSP electron observations and detailed theoretical modeling, will allow us to determine whether the electron distribution evolves in a manner consistent with local acceleration or by inward radial diffusion.

Prompt Acceleration by Drift Resonance New radiation belts have been observed to be created on time scales of minutes, as interplanetary shocks compress the magnetosphere and resonantly accelerate energetic seed populations in the inner magnetosphere (Vampola and Korth 1992; Blake et al. 1992; Wygant et al. 1994). The new belts can persist from months to years after their formation. The mechanism involved is compression of the magnetosphere by an interplanetary shock, which drives a compressional wave deep into the inner magnetosphere. The azimuthal electric fields associated with this shock can be tens to hundreds of $\mathrm{mV} / \mathrm{m}$, on timescales of seconds to minutes. As these compressional waves propagate through the inner magnetosphere, they resonantly accelerate high-energy electrons and protons whose drift periods are commensurate with the wave period (Hudson et al. 1997; Li et al. 1993).

The magnetic field variations in the compressional wave are the direct drivers that energize the seed populations to form the new radiation belt. These quantities must be measured on timescales appropriate to the wave propagation, which requires at least one-second resolution in order to resolve the fast rise time of the initial compressional pulse. At present we have only a basic understanding of this type of event, but with the RBSP mission having two well-instrumented spacecraft at different local times and/or radial distances, we will be able to measure the magnetic and electric field variations at two spatial locations for the first time, and better understand the propagation of the compressional wave. 


\subsection{What Are the Dominant Mechanisms for Relativistic Electron Loss?}

Nearly every magnetic storm begins with a dramatic decrease in relativistic electron fluxes over much of the inner magnetosphere. Some of this drop is the result of reversible adiabatic effects (Kim and Chan 1997), while the remainder represents real loss through wave scattering into the atmosphere, magnetopause shadowing, and demagnetization on highly stretched field lines.

Pitch-angle Scattering and Loss to the Atmosphere Energetic radiation belt electrons can be scattered into the loss cone and lost by collisions in the atmosphere during resonant interactions with whistler-mode chorus emissions (Thorne et al. 2005), plasmaspheric hiss (Lyons et al. 1972; Abel and Thorne 1998a) and electromagnetic ion cyclotron waves (Albert 2003; Summers and Thorne 2003; Jordanova et al. 2008). Although significant advances have been made recently in the theory and modeling of wave-particle scattering (Millan and Thorne 2007), the theoretical calculations need to be thoroughly tested against in situ observations. Our measurements of local electric and magnetic fields and wave power spectral density and angular distribution will enable unprecedented progress in our physical understanding of relativistic electron losses from the inner magnetosphere.

Magnetopause Shadowing and Current Sheet Scattering Electrons can be lost from the radiation belts as they drift through the magnetopause or as they get scattered into the loss cone by current sheet scattering. Both these processes are important at larger L-shells during disturbed geomagnetic conditions, when the magnetosphere is compressed on the dayside and stretched on the nightside. During the main phase of the storm a strong ring current will distort the magnetic field and allow current sheet scattering to move to lower L-shells. Similarly, the outward motion of the radiation belt particles due to the adiabatic effect causes electrons to move to larger L, thus increasing the losses through the magnetopause (Shprits et al. 2006; Jordanova et al. 2008; Loto'aniu et al. 2010). Accurate measurements of magnetic field distortions during geomagnetic storms are required to compute the effectiveness of such loss.

\subsection{How Do Ring Current and Other Geomagnetic Processes Affect Radiation Belt Behavior?}

There are several aspects in which the temporal and spatial evolution of the ring current influences radiation belt dynamics that will be investigated using EMFISIS data. The development of a strong ring current during the main phase of a geomagnetic storm inflates the magnetic field at near-Earth distances. This changes the adiabatic drifts of the charged particles as well as the losses through the magnetopause. Large magnetic field depressions have been measured at distances as small as 3-4 Earth radii $\left(R_{E}\right)$ during major geomagnetic storms (Dst $<250 \mathrm{nT}$ ) and have been associated with the storm time ring current enhancement (e.g., Cahill 1966; Tsyganenko et al. 2003). Sophisticated physics-based models (e.g., Chen et al. 2006a; Jordanova et al. 2006, 2010b; Zaharia et al. 2006, 2010) have been developed to investigate the effect of plasma pressure on the magnetic field in the inner magnetosphere during magnetic storms. The computed magnetic field and electric currents showed that plasma pressure strongly affects the $B$-field, even very close to Earth, and large field depressions develop near Earth at the storm peak. Magnetic field data from EMFISIS will be used to verify these computations and validate the models.

Ring current dynamics are also closely related to the development of intense sub-auroral electric fields involved in the magnetosphere-ionosphere (MI) coupling phenomenon known 
as sub-auroral polarization streams (SAPS). The asymmetric ring current closes via fieldaligned currents through the ionosphere and may be responsible for the penetrating electric fields at mid-latitudes. While the general characteristics of SAPS are well-documented and understood from ionospheric and low-altitude observations (e.g., Foster and Vo 2002; Mishin et al. 2003), the magnetospheric signature has not been as extensively investigated.

During geomagnetic storms ring current distributions are anisotropic and become unstable to excitation of plasma waves which cause the further acceleration or loss of radiation belt particles (see discussion of RBSP objectives in Sects. 2.1 and 2.2). The ion distributions can generate electromagnetic ion cyclotron (EMIC) waves (e.g., Cornwall et al. 1970; Jordanova et al. 1997, 2001b) and magnetosonic waves (e.g., Horne et al. 2000; Chen et al. 2010b), while the electron distributions can excite whistler-mode waves (e.g., Kennel and Thorne 1967; Horne et al. 2003). The wave distributions during various storms will depend on storm strength and ion composition; for example, Thorne and Horne (1997) have shown that increased $\mathrm{O}^{+}$content favors the generation of waves below the $\mathrm{O}^{+}$cyclotron frequency and damps waves above it. Using theoretical modeling (described in Sect. 2.4) together with EMFISIS wave and field observations and RBSP particle measurements, we will identify the evolution of the storm-time ring current and quantify its effects on the radiation belt particles.

EMFISIS will provide measurements of the large-scale magnetic field to place in context the EFW measurements of the convection electric field, an essential quantity for studying ring current evolution during a magnetic storm. Enhanced convection transports moderately energetic particles (ions and electrons) into the inner magnetosphere and accelerates them to form a strong storm-time ring current (e.g., Lyons and Williams 1980; Wolf et al. 1997), while time-dependent variations in the large-scale electric field traps particles on closed drift trajectories (e.g., Ejiri 1978). Kinetic model simulations (Jordanova et al. 2001a, 2003; Liemohn et al. 2001; Zaharia et al. 2010) of ring current development during storms have shown reasonable agreement with the Dst index, indicating the dominant role of magnetospheric convection in ring current energization and trapping. Detailed comparison of modeled ring current distributions, however, showed significant differences at low $L$, depending on the electric field model being used (Jordanova et al. 2003; Yu et al. 2012) and highlight the importance of measuring the large-scale electric field.

Other mechanisms that contribute to ring current flux intensification during the main phase of the storm are radial diffusion (e.g., Chen et al. 1994; Jordanova and Miyoshi 2005) and substorm-induced electric fields (e.g., Wolf et al. 1997; Fok et al. 1999). Radial diffusion affects mostly the local time variations of higher energy ( $>100 \mathrm{keV}$ ) particles, which have drift periods shorter than those of the typical storm main phase and thus drift several times around the Earth during the period of enhanced electric field. Ganushkina et al. (2000) found that plasma sheet ions rapidly penetrate deep into the inner magnetosphere, well inside $L=4$, due to short-lived intense electric fields that are formed in connection with substorm onset. Detailed measurements and simulations are needed to clarify the extent to which these two processes contribute to ring current buildup. EMFISIS measurements of VLF wave properties combined with EFW electric field measurements will clarify the effect of wave-particle interactions and time-varying electric fields on ring current dynamics during geomagnetic storms.

\subsection{Theory and Modeling}

Radiation belt particles are influenced by the global distribution of magnetospheric plasma waves, as well as the global magnetospheric electric and magnetic fields, but the properties 
of such waves and fields will only be monitored along the orbit of the two RBSP satellites. Theory and modeling must be employed to place the spatially-limited observations in a global context. Below, we describe how the EMFISIS theory and modeling team will utilize the available observations to address the primary RBSP science objectives.

Quantifying the Effects of Diffusion on the Radiation Belt Population Measurements made by the EMFISIS wave instruments can only be used to evaluate local rates of radial, pitchangle and energy diffusion. However, over the two-year duration of the mission, statistical models for the global distribution of all relevant waves will be constructed, as a function of MLT, $L$-shell, latitude, and geomagnetic activity. This unique RBSP data source will allow us to develop statistical models for the global distribution of particle scattering, which can be used in a Fokker Planck equation to solve for the temporal variability of phase space density.

Ring Current and Radiation Belt Modeling A newly-developed coupled ring currentradiation belt model (Jordanova and Miyoshi 2005; Jordanova et al. 2006, 2010a, 2010b; Zaharia et al. 2006, 2010) will be used as a powerful tool to understand the dynamics of energetic electrons and ions in the inner magnetosphere. This model represents an extension of our ring current-atmosphere interactions model (RAM) to relativistic energies and electrons. RAM solves numerically the bounce-averaged kinetic equation for $\mathrm{H}^{+}, \mathrm{O}^{+}$, and $\mathrm{He}^{+}$ions and electrons in the Solar Magnetic (SM) equatorial plane and is two-way coupled with a 3-D equilibrium code (SCB) that calculates the magnetic field in force balance with the anisotropic ring current distributions. The electric field model represents the gradient of an ionospheric convection potential (mapped to the SM equatorial plane along SCB field lines) and a corotation potential. The RAM-SCB model can be driven either by empirical electric fields (e.g., Weimer 2001) and boundary conditions or by those provided from a global magnetohydrodynamics (MHD) model, e.g. BATSRUS (Powell et al. 1999) selfconsistently coupled with an electric field model (RIM) (Ridley and Liemohn 2002) and driven by dynamic solar wind input. Figure 3 shows RAM-SCB simulations during the 22 April 2001 storm indicating significant depressions in the magnetic field intensity on the nightside during the storm main phase when the ring current pressure intensifies. The location of the pressure peak, as well as the peak magnitude depends strongly on the strength of the convection and the magnetic field morphology. The EMFISIS measurements of the large-scale electric and magnetic fields will be used to test and improve the physics-based models.

The RAM-SCB model is coupled with a time-dependent 2-D plasmasphere model (Rasmussen et al. 1993). Initially, electron losses due to scattering by plasma waves inside and outside the plasmasphere were included using a simplified loss term $\left(F / \tau_{w p}\right)$ with an appropriate timescale $\tau_{w p}$. Pitch angle scattering by EMIC waves was incorporated, within regions of EMIC instability predicted by the anisotropic ring current ion populations, using quasilinear diffusion coefficients (Jordanova et al. 2008). The diffusion properties of the model will be updated as RBSP data become available. We will perform simulations of geomagnetic storms and compute the electron scattering within the spatial regions of EMIC, magnetosonic, and whistler mode waves using quasilinear theory. This global modeling will allow us to differentiate among the changes of the phase space density from transport and those from local acceleration and loss of energetic particles (RBSP science objectives 1 and 2 discussed in Sects. 2.1 and 2.2).

To assess the changes of the radiation belts due to scattering by various plasma waves, knowledge of the global wave distributions is needed. One of our approaches will be to simulate the wave excitation by the anisotropic ring current distributions using our RAM-SCB 


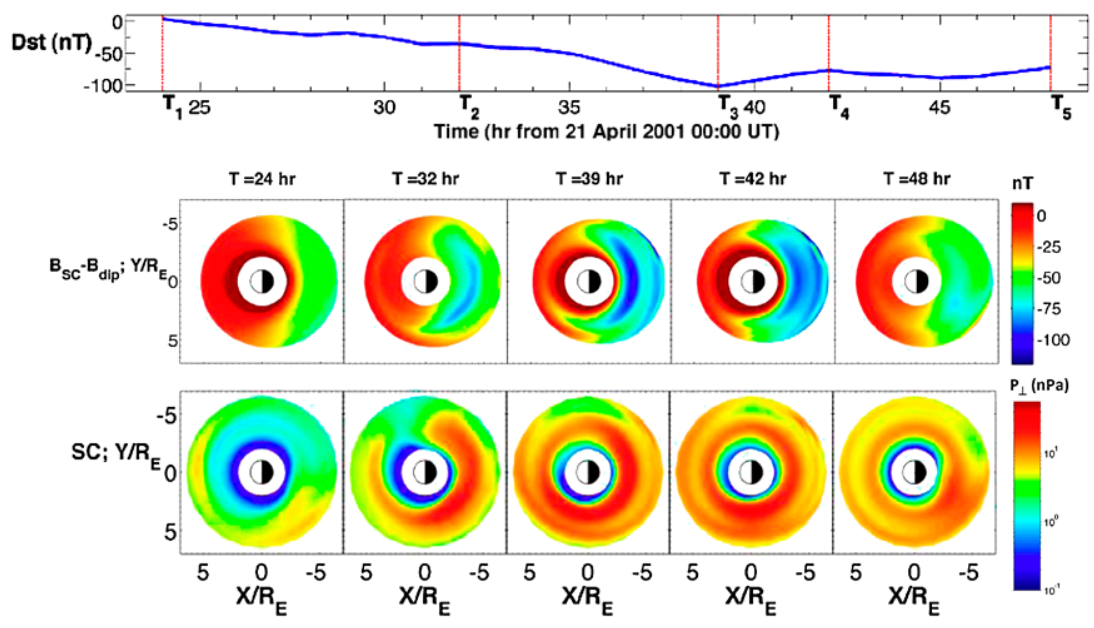

Fig. 3 (Top) Dst index during the April 2001 storm; (Middle) difference between the self-consistently calculated magnetic field intensity and the Earth dipolar field (after Zaharia et al. 2006); (bottom) ring current pressure calculations in the SM equatorial plane with RAM-SCB. Position is given in units normalized to an Earth radius

model (Jordanova et al. 2012) and to compare the wave growth predicted by our model with EMFISIS observations to estimate the wave amplitudes. Another approach will be to use the statistical models for the global distribution of all relevant waves constructed from RBSP data as a function of geomagnetic activity. A valuable test to our model will be provided by comparisons of ion and electron fluxes predicted by RAM-SCB with the pitch-angle distributions measured by the RBSP particle instruments.

Physical Understanding of Wave Excitation To develop a physics-based predictive model of the radiation belts, physical understanding of the most important transport, acceleration, and loss processes is required. Our kinetic ring current model (RAM-SCB) will provide global simulations of the equatorial distribution of all plasma waves important for radiation belt dynamics. We will calculate EMIC and magnetosonic wave excitation by the anisotropic ring current ion distributions during storm time. Figure 4 shows the equatorial growth rate of EMIC waves with frequencies between the oxygen and helium gyrofrequencies obtained with three different model formulations during the November 2002 storm. Intense EMIC waves are generated in the postnoon high-density plasmaspheric drainage plumes by the anisotropic ring current distributions that develop due to drift-shell splitting in realistic nondipolar magnetic fields (Jordanova et al. 2010b). We will perform similar simulations and compare the regions of large wave growth with EMFISIS observations. The global patterns of intense ion and electron precipitation will be compared to energetic particle data from the RBSP mission. In addition, we will simulate with our kinetic model the injection of plasma sheet electrons into the inner magnetosphere by enhanced convection electric fields; this will provide a seed population of electrons. We will calculate the growth rate of whistler-mode waves due to the anisotropic ring current electron population using the dispersion relation for whistler waves and plasmaspheric densities from the coupled plasmasphere model. Global simulations of whistler instability during a geomagnetic storm were performed for the first time by Jordanova et al. (2010a) indicating significant wave growth in the dawnside MLT region outside the plasmasphere. Detailed comparisons with the EMFISIS wave data will be 

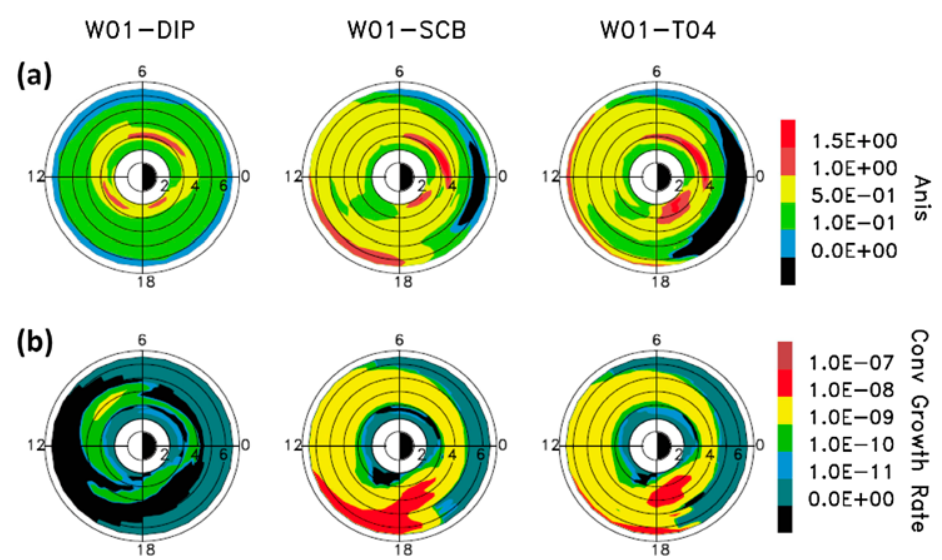

Fig. 4 (a) Proton ring current anisotropy obtained with RAM-SCB at 20 UT 20 November 2002 using W01 electric field and either dipolar (DIP), self-consistent (SCB), or T04 magnetic field, and (b) the corresponding convective growth rate of EMIC waves (after Jordanova et al. 2010b)

made to verify the location of whistler-mode growth and estimate the wave amplitudes. We will investigate the effect of these waves on the local acceleration and loss of radiation belt electrons and compare it to the effects of inward radiation belt transport and acceleration.

Non-linear Wave-Particle Interactions Extremely intense chorus emissions are occasionally observed (Cattell et al. 2008; Tsurutani et al. 2009) with amplitudes (>100 mV/m) far in excess of those where quasi-linear scattering is valid. Non-linear test particle scattering of resonant electrons in such large amplitude waves (Bortnik et al. 2008a) indicates that resonant electrons tend to exhibit advective transport towards the loss cone rather than the stochastic diffusive behavior. Such advective scattering could dramatically increase the average rate of resonant electron loss, and may thus be related to the observed electron dropouts (Onsager et al. 2007; Morley et al. 2010) during the main phase of magnetic storms. Non-linear phase trapping of electrons in large amplitude chorus can also lead to non-diffusive acceleration at relativistic energies (Albert 2002; Furuya et al. 2008; Summers and Omura 2007). Such processes will be treated with test particle scattering codes and the effects will be incorporated into the RAM code simulations.

\section{Suite Overview}

The EMFISIS instrumentation suite provides measurements of DC magnetic fields and a comprehensive set of wave electric and magnetic field measurements (the Waves instrument) covering the frequency range from $10 \mathrm{~Hz}$ up to $12 \mathrm{kHz}$ (to $400 \mathrm{kHz}$ for single-axis electric field) for the RBSP mission. EMFISIS comprises two sensors: a tri-axial fluxgate magnetometer (MAG) and a tri-axial magnetic search coil magnetometer (MSC). Additionally, to measure wave electric fields, the Waves instrument uses signals from the EFW experiment. Signals from these sensors are detected with receivers in a Main Electronics Box (MEB) which collects and processes all of the measurements.

Figure 5 shows a block diagram of the EMFISIS electronics and sensors. The majority of the electronics are contained within the Main Electronics box comprised of seven printed circuit boards. 
Fig. 5 EMFISIS System Block Diagram

Fig. 6 Exploded view of the EMFISIS Main Electronics Box (MEB) showing board positions
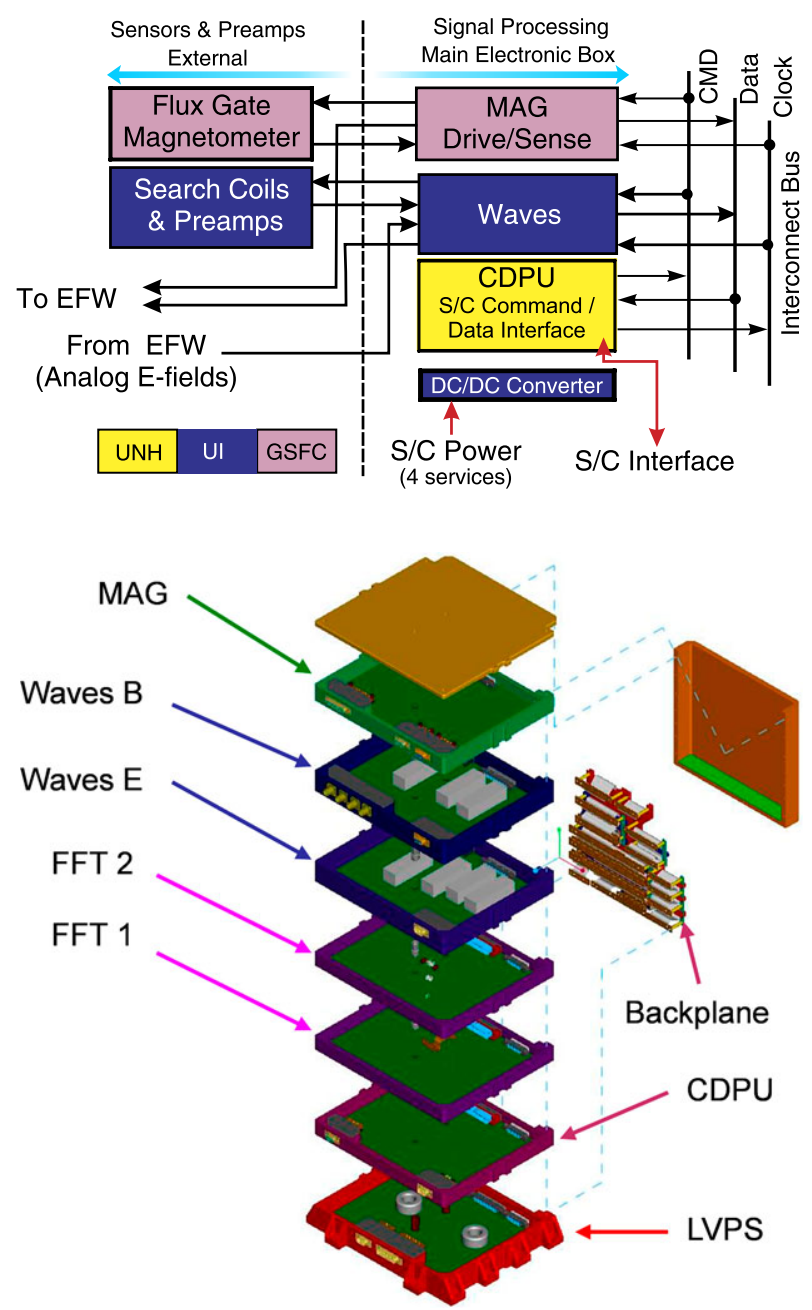

As shown in the exploded view in Fig. 6, from bottom to top, is, first, the Low Voltage Power Supply (LVPS) which converts primary spacecraft power to voltages used by the rest of the suite, followed by the Central Data Processing Unit which controls the suite and handles data transfer to and from the central spacecraft systems. Above this are the four boards of the Waves instrument of the EMFISIS system: two FFT engine boards and the wave electric field and wave magnetic field receivers. Completing the stack is the MAG drive, sampling, and heater control board. Total dose, SEU, and deep dielectric charging issues were carefully considered in the design of all EMFISIS electronics, and these issues are further minimized by placing the MEB deep within the spacecraft bus.

The EMFISIS magnetic field sensors are mounted on booms as shown in Fig. 7. This figure shows the mounting of the various instruments and illustrates the spacecraft $\mathrm{XYZ}$ coordinate system and the UVW scientific coordinate system. All of the field instruments, that is, the EFW electric field booms and EMFISIS MAG and MSC sensors, are nominally mechanically co-aligned with the UVW coordinate system. This has the distinct advantage that phasing between these instruments is more easily verified and the data can be used 


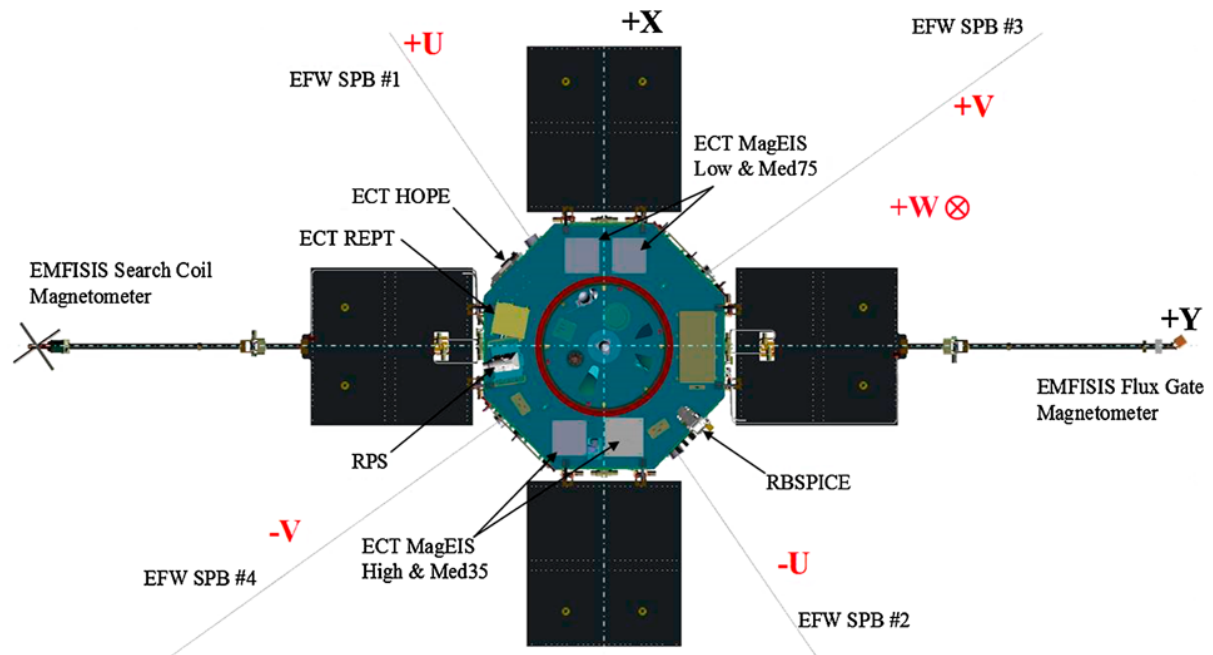

Fig. 7 Bottom view of the RBSP spacecraft layout showing the orientation of the field sensors. Both EMFISIS sensors, MSC and MAG, have their sensor axes nominally aligned with the EFW wire booms. This defines the UVW coordinate system used for analyzing science data. Note that the W direction is into the page. The figure also shows the orientation of the spacecraft XYZ coordinate system

with good success without needing to perform large rotations. However, the final, calibrated EMFISIS data products corrects for any minor misalignments (which less than one degree).

As Fig. 7 illustrates, the EMFISIS magnetic field sensors are mounted on booms which extend from two of the solar panels. These magnetometer booms place the sensors $3 \mathrm{~m}$ from the spacecraft body and a little under $4 \mathrm{~m}$ from the center of the spacecraft. This is done to minimize interference from the spacecraft systems in these measurements. Additionally, the magnetometer boom materials and design have been controlled to minimize or eliminate the use of permeable materials that can produce a magnetic signature. Similarly, the solar panel wiring layout has been controlled to ensure that currents from the solar cell strings cancel and do not produce a significant magnetic signature.

The electric field signals are supplied to the EMFISIS MEB from the EFW experiment. These measurements are made using spherical sensors at the ends of long booms to reduce the effects of the spacecraft at the sensors. In the spin-plane, the sensors are at the ends of wire booms for a tip-to-tip separation of $100 \mathrm{~m}$. Along the spin axis, rigid booms are used which provide a tip-to-tip separation of up to $14 \mathrm{~m}$. The difference in length is included in calculations of the EMFISIS flight software to normalize the signals to each other. Although the length is included, the shorter axial boom on the side of the spacecraft pointing away from the Sun is periodically shadowed (twice per $\approx 5.5 \mathrm{~s} \mathrm{spin}$ ) by the magnetometer booms. This shadowing produces a pulse of approximately $0.3 \mathrm{~s}$ in the $E_{W}$ component of the electric field due to the sudden change in photoelectron current from the probe. The pulse provides low frequency contamination of the survey $E_{W}$ component which must be taken into account when using the data.

\subsection{Details of the Instrument Design}

The science drivers for the EMFISIS design are the need to measure wave properties across the relevant frequency ranges and to measure the DC magnetic field both for background 
field determination as well as to measure very low frequency waves. In both cases this drives the design to measure 3D vector quantities. For waves between $10 \mathrm{~Hz}$ and $12 \mathrm{kHz}$, EMFISIS makes 3D measurements of both the electric and the magnetic field. Below $10 \mathrm{~Hz}$, EMFISIS measures only the magnetic field. However, this measurement can be combined with the 3D EFW electric field data to have a full set of electromagnetic vector quantities. Above $12 \mathrm{kHz}$, only a single electric component is measured from 10 to $500 \mathrm{kHz}$ with decreasing response above $400 \mathrm{kHz}$ due frequency roll-off in the EFW signals.

The highest frequency for the EMFISIS 3D wave measurements is set by the desire to fully measure both lower and upper band whistler-mode chorus. This sets the upper frequency response at $12 \mathrm{kHz}$. The desire to measure the upper hybrid line and the intensity of electron cyclotron harmonics drives the requirement to measure the electric field up to at least $400 \mathrm{kHz}$. A single electric field component is sufficient to satisfy this. Any of the three EFW dipole pairs can be used for the high frequencies, but it is expected that one of the spin-plane dipoles will provide the best sensitivity and lowest noise. The high frequency receiver is designed to measure up to $500 \mathrm{kHz}$, but the signal provided by EFW rolls off, significantly, above $400 \mathrm{kHz}$.

\subsection{Mechanical Design}

The EMFISIS mechanical design is implemented to both support the sensors and electronics for ascent vibration and acoustic loads as well as to provide good thermal conductivity to the spacecraft bus and shielding from penetrating radiation.

The mechanical implementation of the MEB is shown in an exploded view in Fig. 6 which illustrates how the seven boards are stacked and interconnected with a flexible backplane. Each board is contained within its own frame with an EMI cover to prevent crosscoupling of signals between adjacent layers. The boards are also thermally coupled to the frames to provide a conductive heat path for power dissipated on each board. Additionally, connectors are mounted in a "vault" to prevent penetrating radiation from entering through the connectors themselves.

\subsection{Power System}

The Low Voltage Power Supply (LVPS) converts primary spacecraft power to the various DC voltages required by the EMFISIS electronics. The LVPS is implemented as two separate supplies on a single board, one for the analog parts of the system (E and B receiver, MAG electronics) and one for the digital parts of the system (CDPU and FFT engines). This scheme was developed to simplify transformer design as well as to provide resiliency in the event of faults occurring on the digital side of the MEB. If this were to occur, the analog side can be powered separately maintaining MSC and MAG signals that are fed to the EFW experiment, thereby providing a redundant data path.

The analog side of the LVPS provides a set of secondary voltages which are filtered and then regulated by linear regulators to provide very low noise and stable voltages for the analog electronics. Because the power required for these parts of system is quite low, the somewhat lower efficiency of linear regulators is not significant. The digital side of the EMFISIS design consumes the majority of the power and consequently, several radiation hardened switching regulators are used. 


\section{The Fluxgate Magnetometer (MAG)}

The EMFISIS magnetometer on RBSP measures 3D vector magnetic fields essential for our understanding of particle distributions that are critically dependent on the local and global magnetic fields and their time variation. Additionally, the fast measurement capability and wide dynamic range of the MAG together with the MSC search coil allows the detection and measurement of ULF electromagnetic waves that produce particle acceleration and transport in the magnetosphere. The EMFISIS investigation will allow the simultaneous measurement of the ambient magnetic field and its variation at two points within the magnetosphere. This makes possible the development of statistical descriptions of the large-scale dynamics of the magnetic field as well as monitoring the intensity of the quiet and storm-time ring current and the explosive release of energy stored in the geomagnetic tail during substorms. The MAG data also enables comparison of adiabatic invariants and particle phase space density at two points, providing key tests of energetic particle transport in the inner magnetosphere.

\subsection{Heritage}

The EMFISIS magnetometer is the latest in a series of magnetic field investigations developed by the magnetometry group at GSFC. This group has a long and successful track record of development and implementation of complex magnetic field investigations for planetary exploration, earth observing, and space physics missions. The EMFISIS sensors and analog electronics are designs similar to those flown on numerous NASA missions including Juno, MESSENGER, and STEREO. Modifications include changes to the mechanical, power and data interfaces for compatibility with the EMFISIS suite and RBSP spacecraft designs as well as added radiation tolerance through parts selection and shielding. The STEREO instruments were themselves based on more than fifty magnetometers previously developed for space missions, from Voyager (still operational after more than 35 years in space), ISPM, GIOTTO, WIND, ACE, CLUSTER I \& II (more than 10 instruments), DMSP (11 instruments) to the more recent Lunar Prospector and Mars Global Surveyor instruments. They represent state of the art instruments with unparalleled performance.

\subsection{Principle of Operation}

The EMFISIS fluxgate magnetometer is a wide-range, high performance triaxial fluxgate magnetometer system. The signal processing, analog-to-digital converter (A/D) and interface electronics are implemented on a single electronics card shown in Fig. 8 and integrated in the MEB as discussed in Sect. 3. A block diagram is illustrated in Fig. 9. This configuration makes optimal use of limited spacecraft resources and takes full advantage of miniaturization made possible by contemporary technology and the maturity of the magnetometer design.

The wide dynamic range of the instrument covers ambient fields from $0.008 \mathrm{nT}$ to $65,536 \mathrm{nT}$ in three ranges selected automatically by the CDPU or ground command. The upper limit measurement capability is designed to make possible operation and testing in the Earth's surface field and also provides the appropriate range for RBSP perigee measurements. The magnetometer electronics include three 16-bit high resolution A/D converters to easily resolve small amplitude fluctuations of the field. The total power consumption (for zero field) is $\approx 0.9 \mathrm{~W}$ (excluding heater power). High reliability and radiation tolerance is obtained by the use of efficient, conservative design. The principal instrument characteristics are summarized in Table 1. The sensor assembly consists of an orthogonal triaxial arrangement of ring core fluxgate sensors shown in Fig. 10 plus additional elements required for 
Fig. 8 EMFISIS Magnetometer electronics card in its frame

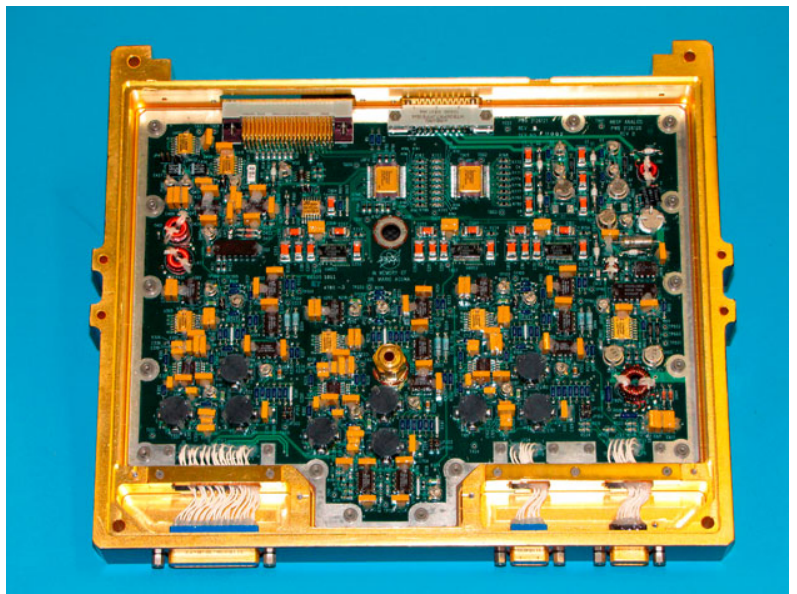

Fig. 9 EMFISIS Magnetometer (MAG) Block Diagram

Table 1 Key Magnetometer (MAG) operating parameters

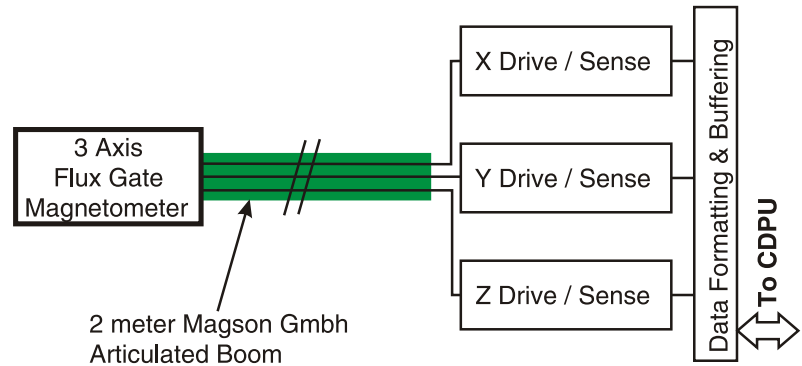

Magnetometer Specifications (three ranges)

\begin{tabular}{ll}
\hline Data Rate & $\sim 3 \mathrm{kbs}$, depending on compression number \\
Sampling Cadence & 64 vectors/s \\
Ranges & Range $3:-65536 \mathrm{nT}$ to $65536 \mathrm{nT}$ \\
& Range $1:-4096 \mathrm{nT}$ to $4096 \mathrm{nT}$ \\
& Range $0:-256 \mathrm{nT}$ to $256 \mathrm{nT}$ \\
& $2 \mathrm{nT}(65536 \mathrm{nT}$ range) \\
Resolution & $0.125 \mathrm{nT}(4096 \mathrm{nT}$ range) \\
& $0.0078 \mathrm{nT}(256 \mathrm{nT}$ range) \\
& $0.1 \mathrm{nT}$ (sensor) \\
Accuracy & $0-30 \mathrm{~Hz}$ \\
\hline
\end{tabular}

thermal control. The fluxgate sensors are the latest in a series developed for space magnetic field measurements by Acuna $(1974,2002)$ with excellent performance and low power consumption. Total mass is $\approx 400 \mathrm{~g}$, including the harness pigtail, cover, and thermal blanket.

The fluxgate sensors are driven cyclically to saturation by a $15.625 \mathrm{kHz}$ signal derived from the CDPU master clock. The sensor drive signals are derived from an efficient high energy storage system which is capable of driving the ring core sensors to peak excitations which are more than 100 times the coercive saturation force of the cores. This type of excita- 
Fig. 10 EMFISIS Magnetometer sensor with cover and connector

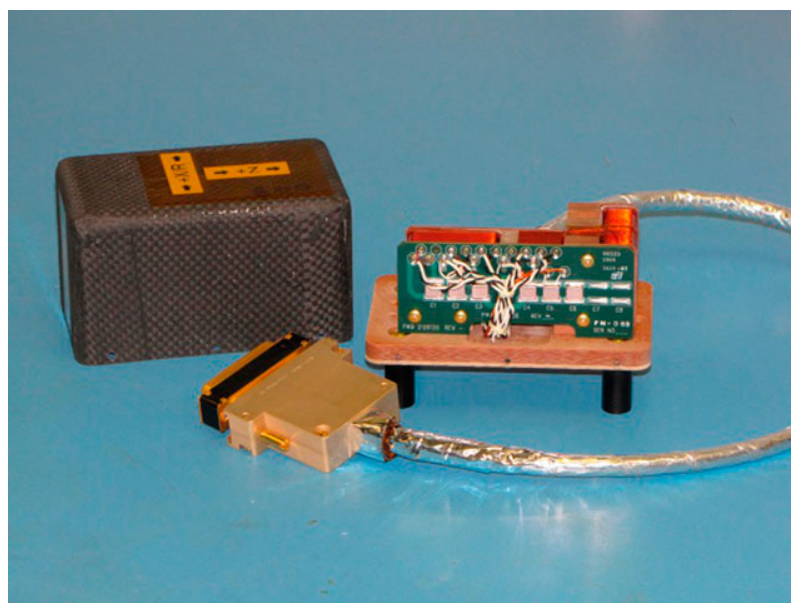

tion eliminates from consideration many "perming" problems which have been attributed to fluxgate sensors in the past. In the absence of an external magnetic field, the fluxgate sensors are "balanced" and no signal appears at the output terminals.

When an external field is applied, the sensor balance is disturbed and a signal containing only even harmonics of the drive frequency appears at the output of the sensors. After amplification and filtering, this signal is fed to a synchronous detector and high gain integrating amplifier used to generate a current proportional to the magnitude of the applied field. This signal is fed back to the sensor to null the effective magnetic field. The output of a single axis magnetometer is then a voltage proportional to the magnitude, direction, and polarity of the ambient magnetic field with respect to the sensor axis orientation. A triaxial magnetometer is created when three single-axis sensors are arranged orthogonally and three sets of signal processing electronics are used to produce three output voltages proportional to the orthogonal components of the ambient magnetic field. For additional information the reader is referred to Ness (1970) and Acuna (1974, 2002).

\subsection{Sensor Functional and Key Design Elements}

The main components of the MAG system are the MEB electronics board, which contains drive, digitization, heater control, and interface circuitry; the MAG sensor which is mounted at the end of a boom; and the harness between the MEB and the sensor.

\subsubsection{MAG Electronics in the MEB}

CDPU Interface The CDPU software tasks perform all required operations: data manipulation and formatting, compression, and packetization for the MAG sensors. All core operations performed by the system are carried out under the control of interrupt driven software synchronized to the telemetry system clock. All default parameter values for the system are stored in tables in CDPU non-volatile memory and mapped into RAM during initialization, and can later be modified by commands to update calibrations, alignments, sampling rates, zero levels, etc. No burst data collection exists for the MAG system; data is always collected at the highest rate possible of 64 vectors/sec. 

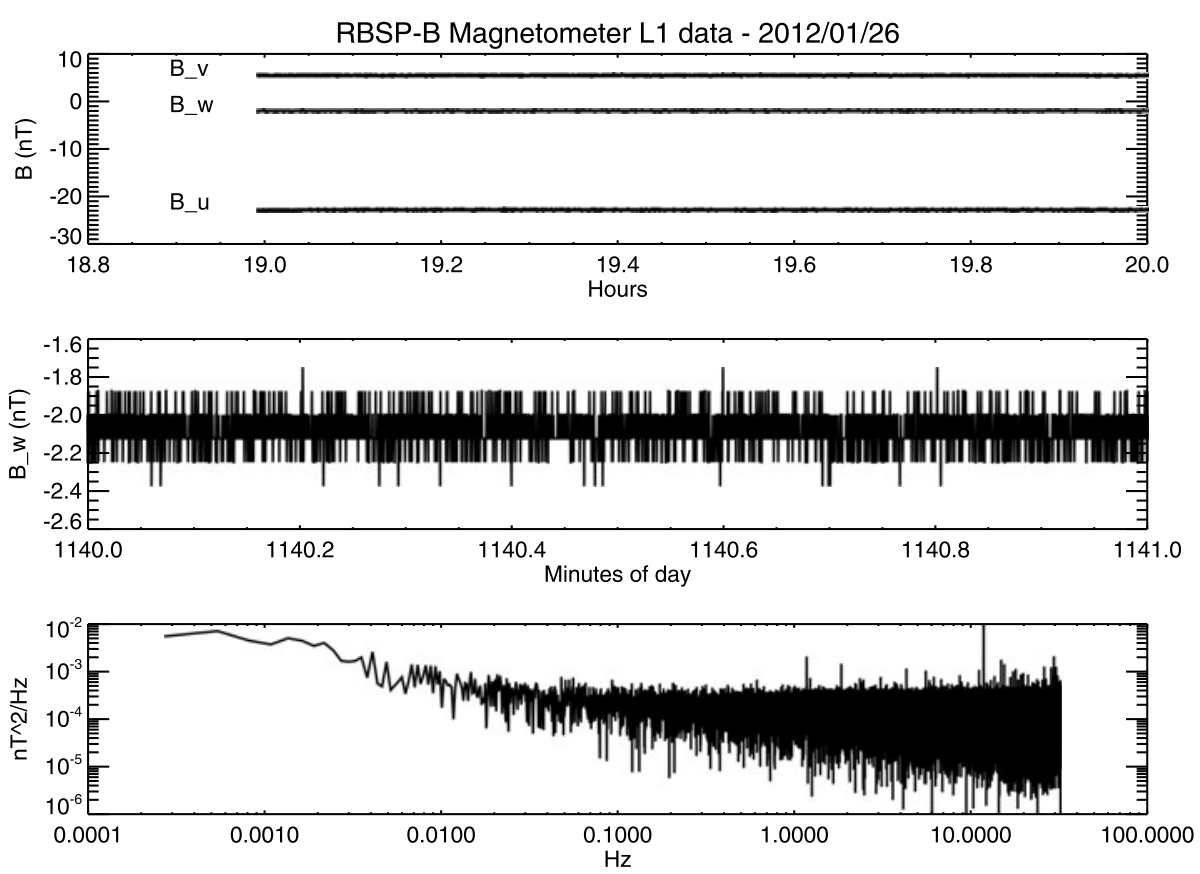

Fig. 11 EMFISIS magnetometer data (RBSP spacecraft B) from the Comprehensive Performance Test of Jan. 26, 2012 while in the \pm 4096 range. Magnetometer data from all three axes (top). The sensor was in a high-quality, mu-metal shield can, so the magnetic field levels are very low. Sixty second data interval from the $\mathrm{W}$ axis sensor (middle). Fluctuations over a range of less than $0.6 \mathrm{nT}$ are due to residual noise from the test environment. Power spectral density of $B_{W}$ data shown in top panel (bottom)

Power and Thermal Control The MAG system is powered by the analog side of the MEB LVPS. There is an additional, isolated power service passed straight through the MEB which provides power to the MAG sensor heater electronics. This power service is only switchable by the spacecraft power system and is not under EMFISIS CDPU control.

To maintain the fluxgate sensors within their optimum operating temperature range, particularly during eclipses (longest is $\approx 24$ hours in duration) on the RBSP orbit, it is necessary to provide heater power to the boom mounted triaxial sensor assembly. This heater prevents thermal cycling of the sensor which could be detrimental to sensor performance as well as maintaining the sensor within the optimum calibrated range.

Since it is extremely difficult to reduce the stray magnetic field associated with the operation of DC powered foil heaters to acceptable levels, a pulse width modulator operating at $\approx 50 \mathrm{kHz}$ is used to obtain automatic, proportional control of AC power supplied to the heating elements. The nominal power required to maintain the sensors at the desired temperatures is determined by modeling to lie in the range of 0.40 to 0.55 watts.

\subsubsection{MAG Sensor Response and Characteristics}

The performance of the MAG fluxgate sensors is shown in Fig. 11. Total RMS noise level over the $0.001-10 \mathrm{~Hz}$ band does not exceed $0.01 \mathrm{nT}^{2} / \mathrm{Hz}$. This noise level is well below the required sensitivity for the RBSP mission and is more than adequate to properly detect and identify all magnetic field phenomena of interest. 
The MAG system uses a single-pole low-pass filter with a $3 \mathrm{~dB}$ point at $30 \mathrm{~Hz}$ on all three axes. This provides a gentle roll-off for anti-aliasing and removes any differences between sensor cores as a function of frequency. The nominal operating temperature of the sensor and electronics is $\approx 15{ }^{\circ} \mathrm{C}$ and this has been achieved in flight. Very little temperature variation is experienced by the MAG electronics which are inside the spacecraft and essentially no variation is seen across the three MAG ranges. The sensor heater maintains the sensor within a few degrees of the nominal temperature, well within its well-calibrated range.

The three analog signals generated by the magnetometer are digitized by the $16 \mathrm{bit} A / \mathrm{D}$ converter. The 16 bit resolution allows the recovery of a very large dynamic range of signals. To further increase the measurement dynamic range and to accommodate simplified integration and test requirements during spacecraft testing, the dynamic range of the magnetometer can be changed automatically if the magnitude of the measured signals exceeds or drops below established, programmable digital thresholds. In this fashion, the MAG instrumentation can cover seven orders of magnitude in magnetic field measurement capability, from $0.008 \mathrm{nT}$ to $65536 \mathrm{nT}$ per axis. The operation of the automatic ranging system is controlled by the EMFISIS CDPU and has hysteresis to prevent overly frequent range changes. It is not expected that the $256 \mathrm{nT}$ range will be used because the Earth's field only falls into this range near apogee and is quite variable. This would likely result in frequent range changes for geomagnetically interesting times which is not desirable. Consequently, range changes will typically occur only twice per orbit, once on the outbound leg as the Earth field drops and once on the inbound leg as the field increases. These range changes occur at approximately $2 R_{E}$ where the Earth's equatorial field decreases below $4000 \mathrm{nT}$.

Data compression is used in the CDPU to reduce the "raw" data rate (64 samples/s) to a value compatible with spacecraft resources and science objectives and is described in more detail in the section on the CDPU. The CDPU also controls calibration sequences that provide the necessary currents to determine the scale factor of each of the magnetometer axes for the two dynamic ranges.

\subsection{Calibration}

The MAG instrument calibration involves several traditional steps that were performed first at the laboratory level and later at the GSFC magnetics test facility. Initial calibrations are performed during electronics tuning and adjustment, and after each environmental test. The instrument incorporates a high accuracy internal calibration source that allows monitoring of trends or anomalies in performance in an end-to-end fashion. After the electronics and sensors are fully integrated a high accuracy scale factor and alignment test is performed using an absolute standard proton precession magnetometer. Parameters such as frequency response, zero levels, analog-to-digital conversion calibration and calibration sources are established with high accuracy in the laboratory using high accuracy sources and magnetic shields.

The spacecraft magnetic field signature is required to be less than $5 \mathrm{nT}$ at the sensor. Prior to launch, this is verified by performing a "swing test" of the spacecraft in which the spacecraft is hung from a hoist and very gently swung back and forth in a direction aligned with a pair of external magnetometers. These magnetometers are operated in "gradient" mode which removes much of the background Earth field and allows the identification of the oscillating field from the swinging spacecraft.

The alignment of the sensor to the spacecraft reference is first done via control of the mechanical tolerances of the mounting and boom and allows an alignment determination of $\approx 1^{\circ}$. Final calibration and alignment analyses are performed in flight. A spinning spacecraft is an ideal platform to determine, from the modulation of the ambient field, the precise 
value of the alignment of the spin axis to the magnetometer as well as the axes parallel to the spin plane. In addition, the spin-modulated magnetic signal allows the estimation of spin plane zero levels to an accuracy approaching the noise level of the measurements. Final calibration and alignment, now underway, is expected to be better than $\approx 0.1 \mathrm{nT}$ and $\approx 0.1^{\circ}$ respectively. Details of how the calibrations are applied to the magnetometer data are given in Sect. 8.1

\section{The Waves Instrument}

The primary objective of the Waves instrument is to provide sufficient information on plasma waves in the radiation belts to quantitatively determine the effect of these waves on radiation belt particles. Specifically, the Waves instrument measures all 3 components of the electric and all 3 components of the magnetic field for waves in the frequency range between $\approx 10 \mathrm{~Hz}$ and $12 \mathrm{kHz}$. The basic data returned is a set of spectral matrices including the auto- and cross-correlations between the sensors which, either on the ground or in the CDPU allow for the determination of spectral densities and various wave propagation characteristics. In burst mode, a primary data set includes full digitized waveforms from all six sensors to enable all types of wave analyses to be performed on the ground for a select fraction of times. The Waves instrument in concert with the CDPU also includes the ability to measure propagation characteristics of the waves such as the wave normal angle and Poynting flux for electromagnetic waves such as whistler-mode hiss and chorus within the constraints of limited telemetry. Measuring both the electric and magnetic components of the waves also allows one to distinguish between electromagnetic and electrostatic waves.

The instrument also measures a single electric field component of waves from $10 \mathrm{kHz}$ to $500 \mathrm{kHz}$ (with limited response above $400 \mathrm{kHz}$ due to roll-off of the EFW signals) in order to determine the spectrum of electron cyclotron harmonic emissions and to measure the frequency of the upper hybrid resonance band, thereby providing an accurate determination of the electron density. Any of the three EFW dipoles can be selected by command to do this, but typically it is one of the spin plane booms will be used for optimum sensitivity upon evaluation in orbit. Figures 12 and 13 show the frequency range and amplitudes for the wave phenomena relevant to the RBSP objectives. The Waves instrument has a suitable dynamic range to cover all of these wave phenomena.

The Waves instrument also includes a set of four FPGA-based floating point accelerators called FFT engines to allow efficient digital signal processing of the Waves measurements onboard. The FFT engines can be considered computational resources controlled by the Leon III CDPU which assigns tasks to the engines and collects the results when the tasks are complete, as signaled by an interrupt. The multiple engines allows the CDPU to orchestrate complex modes requiring various computational tasks to be performed in concert or independently without having to wait for this resource to become available. While the engines are optimized for floating point fast Fourier transforms, they also control the Waves analog-to-digital converters to acquire waveforms; can Rice compress waveforms for transmission to the ground for burst modes; can calibrate, bin and average spectra; and be used to compute spectral matrices.

\subsection{Waves Sensors}

The Waves magnetic sensors consist of three identical search coil antennas mounted in a tri-axial configuration with each antenna oriented parallel to one of the spacecraft scientific 
Fig. 12 Comparison of electric field wave phenomena important for RBSP science objectives with EMFISIS Wave frequency coverage and noise level measured during ground testing. The noise level at the lower frequencies is better in orbit due to the absence of $60 \mathrm{~Hz}$ interference

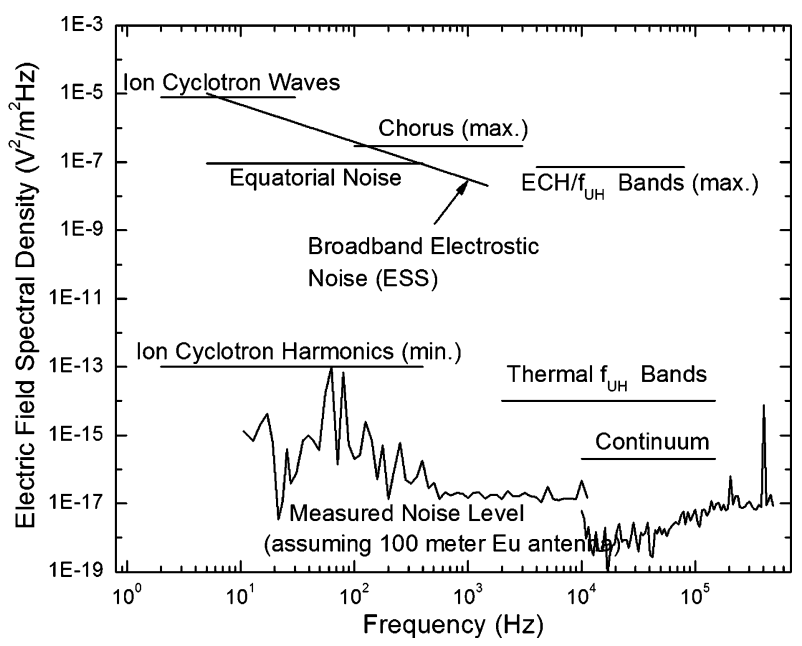

Fig. 13 Comparison of magnetic field wave phenomena important for RBSP science objectives with EMFISIS Wave frequency coverage and noise level

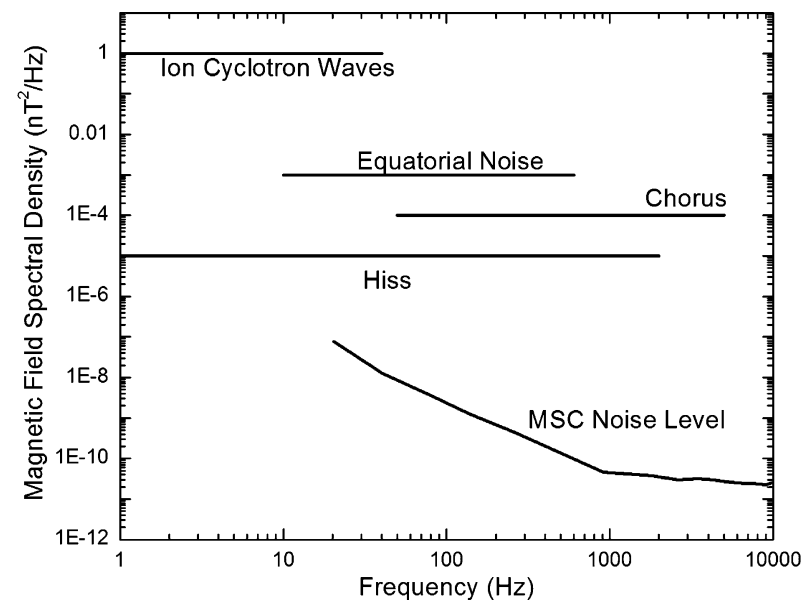

(UVW coordinates) axes. Two are parallel to the two spin-plane EFW electric field double probes and the third is parallel to the spin axis double probe. The search coils are mounted on the boom opposite from the magnetometer boom approximately $3 \mathrm{~m}$ from the spacecraft body to reduce any interference from the spacecraft. To enable the measurement of small amplitude signals, the spacecraft systems and other instruments have been designed and built with sound engineering practices that minimize electromagnetic interference.

The electric field signals for the Waves instrument are provided to the EMFISIS instrumentation from the EFW experiment. These signals consist of differential voltages from opposing EFW spherical sensors. Two of these signals, $E_{U}$ and $E_{V}$, are derived from long wire booms in the spin-plane of the spacecraft. The third signal $E_{W}$ is provided by rigid booms aligned along the spacecraft spin axis. Likewise, Waves provides analog signals from the triaxial search coils to the EFW instrument.

The magnetic search coil (MSC) design is based on previous sensors designed and built at the University of Iowa, including those for Hawkeye, ISEE, DE, CRRES, Polar, Wind, and Juno. Each search coil utilizes a mu-metal core approximately $40 \mathrm{~cm}$ in length and is wound with 10,000 turns of wire. The MSC sensors have a usable frequency range from 
Fig. 14 The Magnetic Search Coil (MSC) sensor assembly and preamplifiers

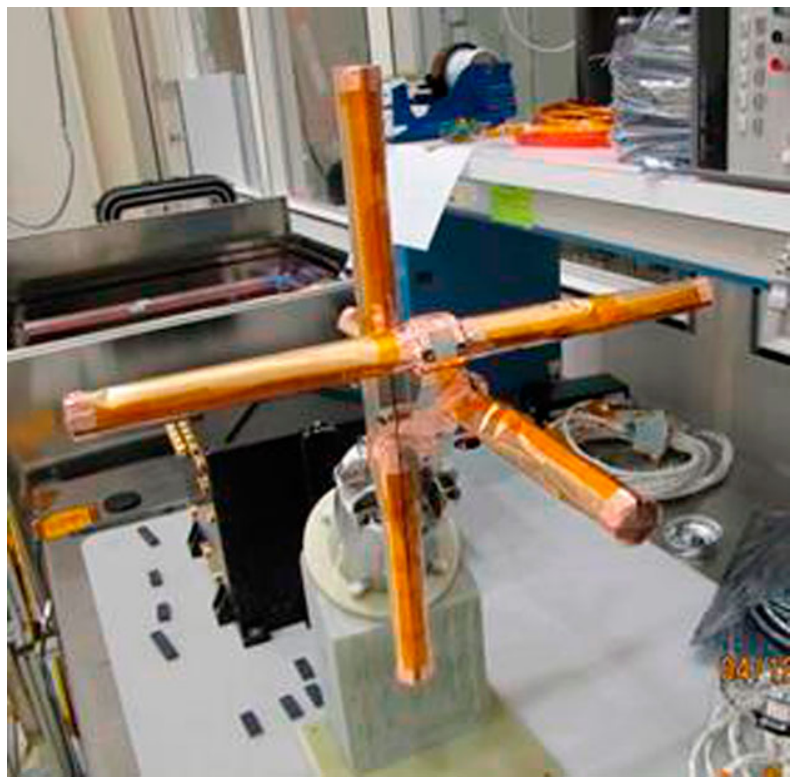

below $10 \mathrm{~Hz}$ to $12 \mathrm{kHz}$. A flux feedback system is used to flatten the frequency response over the range of about $30 \mathrm{~Hz}$ to $12 \mathrm{kHz}$ to provide a more easily calibrated output. The transfer function in this range is about $1.2 \mathrm{~V} / \mathrm{nT}$. The preamplifiers are mounted in a housing adjacent to the sensors in cylindrical housings on the end of the MSC boom. The search coils and preamplifiers are designed to operate within calibration between -70 and $+30{ }^{\circ} \mathrm{C}$ and with a thermal design which does not require heaters during operation. A survival heater is provided for periods when the sensor is turned off. The MSC preamplifiers are specifically designed with radiation tolerant parts and spot shielding so as to operate in the RBSP radiation environment. The same design is also used on the Juno mission headed to Jupiter. A photo of the RBSP search coil and preamplifier assembly is shown in Fig. 14.

\subsubsection{MSC Calibration and Performance}

The calibration philosophy for the Waves instrument was to first calibrate the receivers and sensors individually, then perform a calibration of the combined sensors and receivers system (end-to-end), and verify that the results match.

The MSC sensor coil and pre-amplifier sub-systems were each calibrated (amplitude and phase from $\approx 1 \mathrm{~Hz}$ to $\approx 15 \mathrm{kHz}$ ) in a single axis mode just prior to assembly in the threeaxis housing using a solenoid calibration coil inside a cylindrical mu-metal shield (to reduce $60 \mathrm{~Hz}$ interference). The calibrations were performed at the expected maximum temperature extremes of the sensors $\left(-70\right.$ to $\left.30^{\circ} \mathrm{C}\right)$ and at room temperature $\left(+22{ }^{\circ} \mathrm{C}\right)$. Following final assembly of the MSC sensors and preamplifiers into the tri-axial configuration (see Fig. 14), the MSC units were re-calibrated using a three-axis square stimulus coils in a large square mu-metal shield box to verify that the calibration had not changed. Because of the size of the tri-axial sensor unit and the square mu-metal shielding box, these tests were only performed at room temperature $\left(+22{ }^{\circ} \mathrm{C}\right)$.

The tri-axial MSC unit was then attached to the EMFISIS MEB and the calibration tests were repeated to measure the response of the complete system (sensors + receivers). After 
Fig. 15 The transfer function for each of the six single axis search coil sub-assemblies. As can be seen, the six units are nearly identical, allowing the use of a single table to calibrate the gain of the sensors

Fig. 16 The measured noise level in the lab at $22{ }^{\circ} \mathrm{C}$ of the six single-axis search coil sub-assemblies
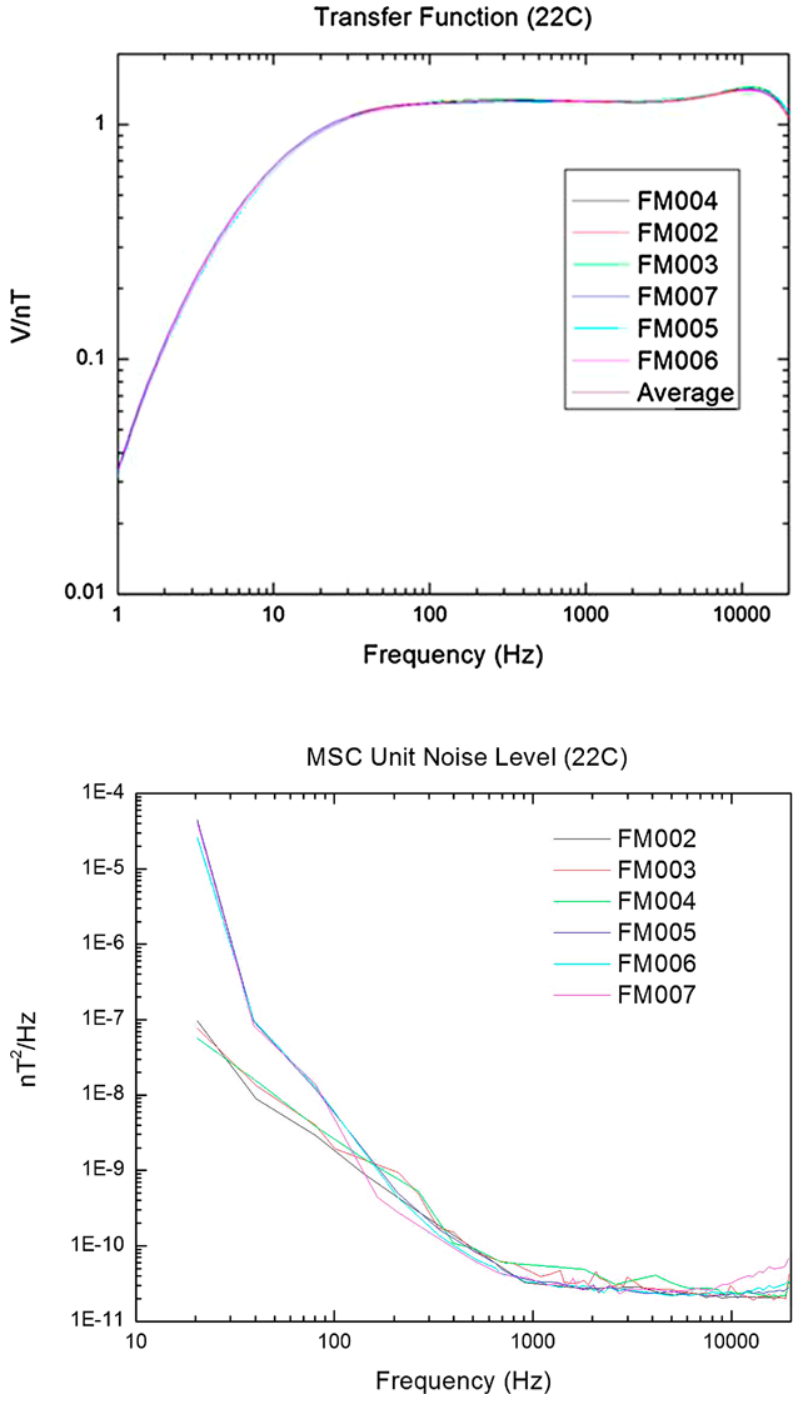

delivery to the spacecraft the calibrations were repeated using the flight harness. During a series of interface tests with the EFW instrument, the calibrations were again repeated to verify the interfaces between the EMFISIS and EFW instruments.

Figure 15 shows the resulting transfer functions of all six of the individual, single-axis MSC sense coil and preamp sub-assemblies as well as the average of all six. As can be seen, the six units are very similar (within a fraction of a dB).

Figure 16 shows the Noise Levels measured for of all six MSC units. The difference between the units for Spacecraft A (FM 2, 3, \& 4) and Spacecraft B (FM 5, 6, \& 7) below about $100 \mathrm{~Hz}$ is due to a change in the test setup which provided better grounding of the test equipment during the testing of the units for Spacecraft $A$ and a decrease in background $60 \mathrm{~Hz}$ noise, and not due to a differences between the sensors. The in-flight noise levels at low frequency are better than is shown here. 


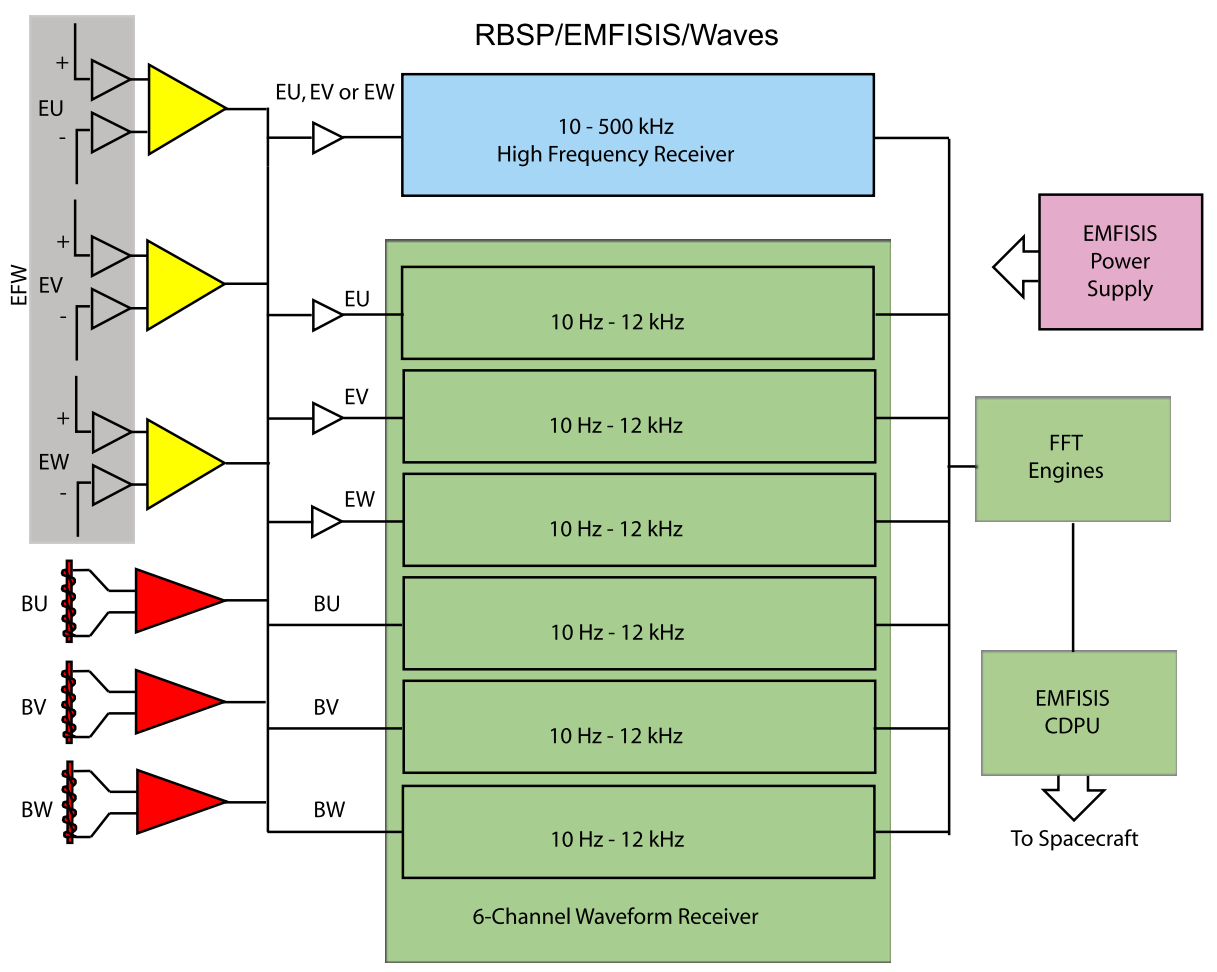

Fig. 17 Waves System Block Diagram

\subsection{Waves Receivers}

Figure 17 shows a block diagram of the Waves instrument. The six inputs from the sensors are shown on the left. In the middle, the various receivers are shown. The six receivers are identical waveform receivers with flat (to within $1 \mathrm{~dB}$ ) response from $10 \mathrm{~Hz}$ to $12 \mathrm{kHz}$. Together, they constitute the six-channel Waveform Receiver (WFR). Each receiver consists of a variable gain amplifier followed by a bandpass filter $(10 \mathrm{~Hz}-12 \mathrm{kHz})$. A 16 bit A/D converter is used to digitize each signal at a sampling rate of $35 \mathrm{kHz}$.

The primary output of the WFR is a set of six phase-matched waveforms which can be processed in various ways. These continuous waveform samples of a selectable (of up to 6 s) duration can be losslessly compressed by a factor of between 2 and 4 in the Waves FFT Engine coprocessors and stored for transmission to the ground.

The waveform time series comprise the primary burst mode of the Waves instrument. These data can be used for spectrum analysis, spectral matrices, wave normal analysis, and Poynting flux calculations on the ground. Second, the waveforms can be analyzed in the Waves FFT Engine coprocessors to produce spectral matrices similar to those computed on the Cluster STAFF instrument (Cornilleau-Wehrlin et al. 2003) which can be generalized to other instances (Santolik et al. 2001).

The spectral matrix is generated from $468 \mathrm{~ms}$ waveforms captured every $6 \mathrm{~s}$ and is telemetered to the ground as a regular survey data product, allowing computations of wave normals, Poynting flux, and other propagation characteristics. Another burst mode provides a set of spectral matrices every $30 \mathrm{~ms}$, commensurate with the typical time scale of discrete 
chorus elements. All of these data sets include spectral densities as a function of frequency. These can be losslessly compressed and stored for transmission to the ground. These data provide information that will be used for determining the effectiveness of the waves in acceleration, heating, and pitch-angle scattering of radiation belt particles.

The top receiver in the middle of the block diagram in Fig. 17, the High Frequency Receiver (HFR) is designed to provide spectral information from any one of the three EFW electric dipole antennas for the frequency range from $10 \mathrm{kHz}$ to $500 \mathrm{kHz}$. Typically, one of the spin-plane pair is be used and will be selected on the basis of optimal noise level and sensitivity once on orbit. The HFR consists of a bandpass filter, followed by a variable gain amplifier with two discrete gain settings based on the input signal strength and a 14-bit A/D converter. The bandpass filter covers the range from 10 to $500 \mathrm{kHz}$, acting as an antialiasing filter at $500 \mathrm{kHz}$. The effective upper frequency response is limited to $400 \mathrm{kHz}$ by the response of the EFW pre-amps driving the series resistance and capacitance of the long cables which connect them to the EFW electronics on the spacecraft. Digitized waveforms from this channel are spectrum analyzed by the digital signal processing electronics in the Waves FFT engines. It is also possible to compress and pass along the waveforms as a burst mode option for storage and eventual transmission to the ground, if desired.

In the plasmasphere and along the plasmapause this high frequency receiver will measure the frequency of the upper hybrid resonance band at $f_{u h}$ which, in turn, provides an accurate measure of the electron plasma frequency, hence, electron density by $n_{e}=\left(f_{u h}^{2}-f_{c e}^{2}\right) / 8980^{2}$ where $n_{e}$ is in $\mathrm{cm}^{-3}$ and the frequencies are in $\mathrm{Hz}$. The electron density is a crucial plasma parameter for modeling and ray tracing. It is also most easily and accurately measured by means of resonances and cutoffs in the wave spectrum rather than particle detector measurements which are subject to spacecraft charging and other complicating factors. Beyond the plasmapause where the $f_{u h}$ band is less distinct or absent, the low-frequency cutoff of nonthermal continuum radiation can be used to determine $f_{p e}$. The Waves instrument has the capability to determine the electron density along the spacecraft trajectory where densities are $2000 / \mathrm{cm}^{3}$ or lower which covers much of the RBSP orbit. The EFW investigation's spacecraft potential measurements will also provide electron densities, especially at lower altitudes where the electron densities exceed $2000 / \mathrm{cm}^{3}$. The two techniques are complementary and provide an excellent set of tools by which to make this very important measurement.

The primary output of the HFR will be spectral information at a rate of one spectrum every $6 \mathrm{~s}$. However, it is possible to return higher cadence spectra as well waveforms in a burst mode.

\subsubsection{Waves Receiver Calibration and Performance}

An extensive series of calibrations and instrument performance checks were carried out on the EMFISIS Waves receivers, both before and after integration on the spacecraft. The Waves receivers were calibrated both individually and combined with the MSC sensors (endto-end) at the University of Iowa. After delivery to the spacecraft, a series of interface tests were performed with the EFW instrument to verify the electrical performance and calibrations through the EFW antenna and preamplifiers, and also through the MSC sensors to EFW. These end-to-end calibrations were compared to the individual sensors and receiver calibrations to verify that the results matched. For both the MSC and the EFW signals, essentially the same end-to-end frequency and phase response was found. However, the EFW axial signal does have a smaller separation and consequently the effects of coupling to the plasma can lead to different response depending on the plasma environment.

Amplitude calibrations for each of the receivers were accomplished by providing an input signal of fixed frequency. The amplitude of the stimulus was decreased in $2 \mathrm{~dB}$ increments to 

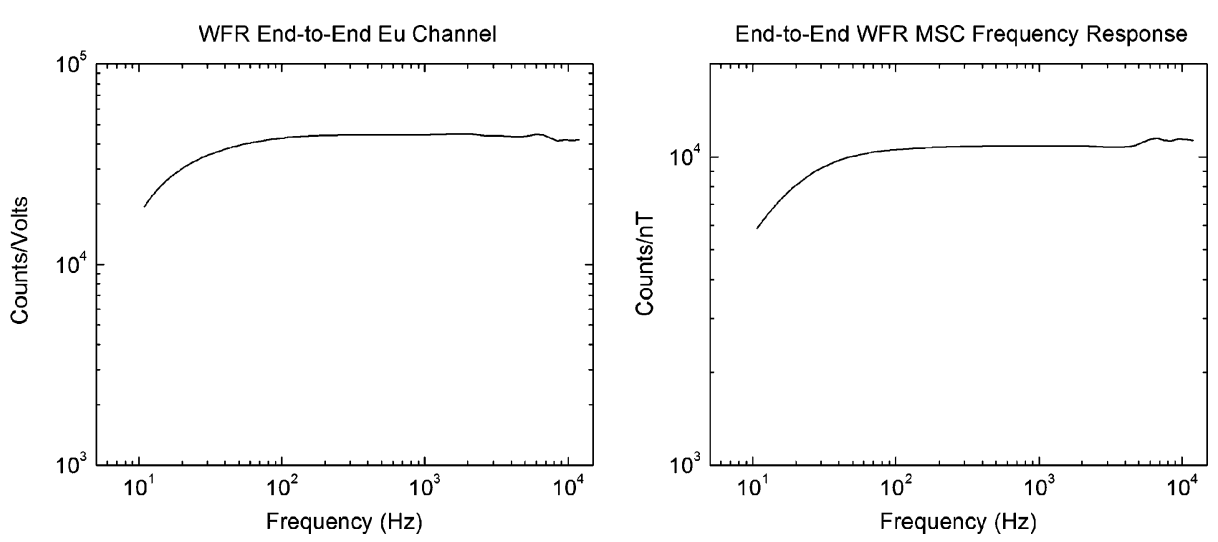

Fig. 18 WFR Electric and Magnetic Channel Frequency Response

cover the full amplitude range of the receiver. Amplitude calibrations were also performed with an input of white noise that was flat over the frequency range of the receiver. Frequency and phase calibrations were accomplished by sweeping an input signal of known amplitude and phase over the frequency range of the receivers. For the WFR receivers, additional calibrations were performed by applying the same white noise and a pseudo-random noise signal to the six receivers. These calibrations are used to construct lookup tables that convert the telemetry data value to the true input signal strength and phase.

Six-Channel Waveform Receiver Calibration The response of the six-channel waveform receivers was determined by applying signals of known frequency, amplitude and phase (sine waves, white noise, and pseudo-random noise) to the receiver inputs, and determining the gain factors required to convert the telemetry values into physical units. These gain factors provide calibrations for the time-series waveform, the onboard spectral matrix calculations, and the spectrum produced by a Fourier transform of the waveform on the ground. The amplitude and frequency response of the six-channel waveform receivers was determined for each attenuator state, and for the two EMFISIS to EFW MSC filter modes. End-to-end calibration checks were performed by repeating the frequency response test and by applying an input signal of white noise with known spectral properties to the input of the electric and magnetic preamplifiers. Figure 18 shows the average frequency response of the three electric (left panel) and three magnetic (right panel) channels of the six-channel waveform receiver for both spacecraft.

High Frequency Receiver Calibration The response of the HFR receiver was determined by applying signals of known frequency and amplitude (both sine waves and white noise) to the receiver inputs, and determining the gain factors required to convert the telemetry values into physical units. These gain factors provide calibrations for the time-series waveform, the onboard spectrum, and the spectrum produced by a Fourier transform of the waveform on the ground. The amplitude response of the HFR receivers was determined for each attenuation state. End-to-end calibration checks were performed by repeating the frequency response test and by applying an input signal of white noise with known spectral properties to the input of the EFW electric preamplifiers and to the MSC sensors. Figure 19 shows the average frequency response of HFR receiver. 
Fig. 19 HFR Frequency

Response

Fig. 20 WFR and HFR Noise Level ( $E_{U}-100$ meter antenna)
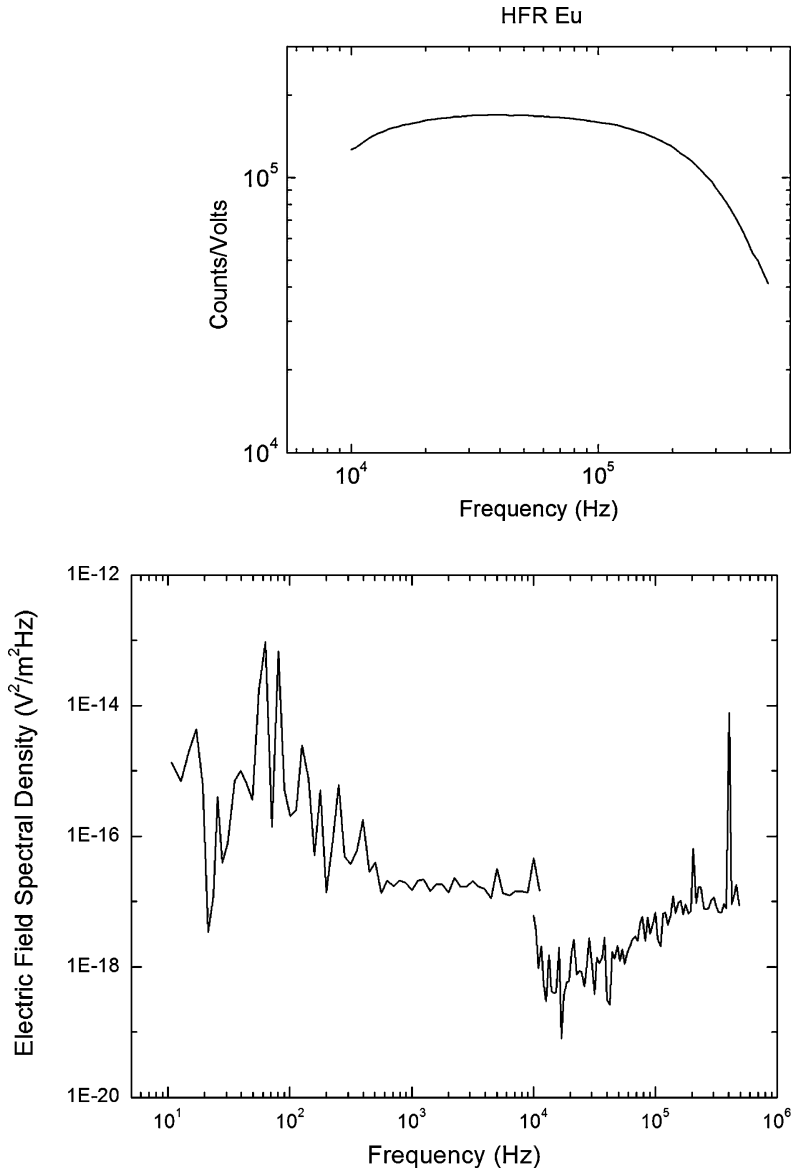

WFR and HFR Receiver Performance Figure 20 shows the noise level of the WFR and HFR receivers attached to the $E_{U}$ antenna and assuming an antenna length of 100 meters that was obtained on the spacecraft during interface testing with EFW. The noise lines at lower frequencies are believed to be primarily interference from GSE equipment $(60 \mathrm{~Hz})$. The overall noise level is much better in flight. The noise line at $400 \mathrm{kHz}$ is a power supply interference line from EFW.

\subsection{Waves FFT Engines}

The Waves instrument includes four custom designed digital signal co-processors to address the large amount of computationally intensive signal processing required, called the Waves FFT Engine (WvFE) co-processors. These co-processors have been implemented in an Actel field programmable gate array (FPGA) that provides a high level of radiation tolerance, high performance and low power consumption. Nearly identical implementations of this co-processor design have been flown on the Juno Waves investigation as well.

The WvFE co-processors work in tandem with the CDPU, whom manages the scheduling of tasks across the co-processors and reads back the data produced by these tasks. The CDPU performs this management by selecting an available co-processor from the set, writing a task descriptor to the selected co-processor, and then waits for an interrupt to signal the 
completion of the task. Once an interrupt is received, the CDPU will read back the results produced by the co-processor and then take subsequent action.

The logical design of the WvFE co-processor is described in a hardware description language (HDL) as a 'system on a chip', where multiple cores (logic elements) are connected together via an internal on-chip bus fabric. These cores are responsible for a number of tasks including the collection of science data, application of digital signal processing techniques and the compression of waveform data. All of the cores in a co-processor are able to access a shared working memory, known as waveform memory, through the on-chip system bus fabric. The waveform memory is used as a storage area for captured waveforms, program code, and partial/final products. The waveform memory is eight megabytes in size and is specific to a co-processor, where each co-processor has its own independent complement of memory to increase overall system bandwidth.

One type of core found in the WvFE co-processor, the A/D controller, is responsible for capturing and pre-processing waveform data. Each co-processor interfaces with the six $\mathrm{ADCs}$ on the waveform receiver and a single ADC on the high frequency receiver through its set of $\mathrm{A} / \mathrm{D}$ controllers. To capture a waveform, an $\mathrm{A} / \mathrm{D}$ controller core is programmed with the needed size and sample rate of the capture, once initiated the controller begins to sample the waveform channel(s) and converts each sample to a two's complement numbering notation. These pre-processed samples are saved in the co-processors' waveform memory.

Once waveform data is available, several other cores in the WvFE co-processor can now be used to further process the waveform. A Rice compression core capable of losslessly compressing 12-bit or 16-bit integer data provides a method for compressing raw waveforms. To apply signal processing techniques to the captured waveform, the UI's custom designed DSP processor architecture will be used, this is called the Waves FFT Engine core.

The $\mathrm{WvFE}$ core is a programmable general-purpose digital signal processor that performs DSP calculation in IEEE 754 single precision floating point arithmetic. The processor is capable of executing two instructions per clock cycle with at a peak performance of 21 million floating-point operations per second (MFLOPS) and 10.5 million integer operations per sec (MIPS) at $21 \mathrm{MHz}$. The processor's floating-point unit has been implemented as a multiplyaccumulate operation that greatly aides in the acceleration of common vector calculations. Several signal processing algorithms have been implemented in programs specific to the WvFE core's instruction set architecture, including waveform windowing, the fast Fourier transform, spectral average and binning, as well as spectral matrix computations. Given the programmable nature of the WvFE core architecture the software executed by the processor defines how it operates. This allows future updates to the software to extend or modify the DSP capabilities of the instrument.

In operation, the WvFE core sets up and then executes the digitization of a set of waveforms, controlling the analog to digital converters on the appropriate receiver board(s). The digitized waveforms are then processed by first applying a Hann window and then the FFT operation. The calibration, in both phase and amplitude, is then applied to the spectral components which are summed into bins (based on a programmable binning table). Following this, the complex cross-multiplication is performed and the spectral matrix and/or the waveforms are made available to the CDPU for transmission to telemetry.

\section{The Central Data Processing Unit (CDPU)}

The CDPU manages coordinated electromagnetic field data for the entire set of EMFISIS instruments: controlling the intra-instrument timing, the data collection scheme and the interface to the spacecraft data system. Time series data from MAG and Waves, as well as 


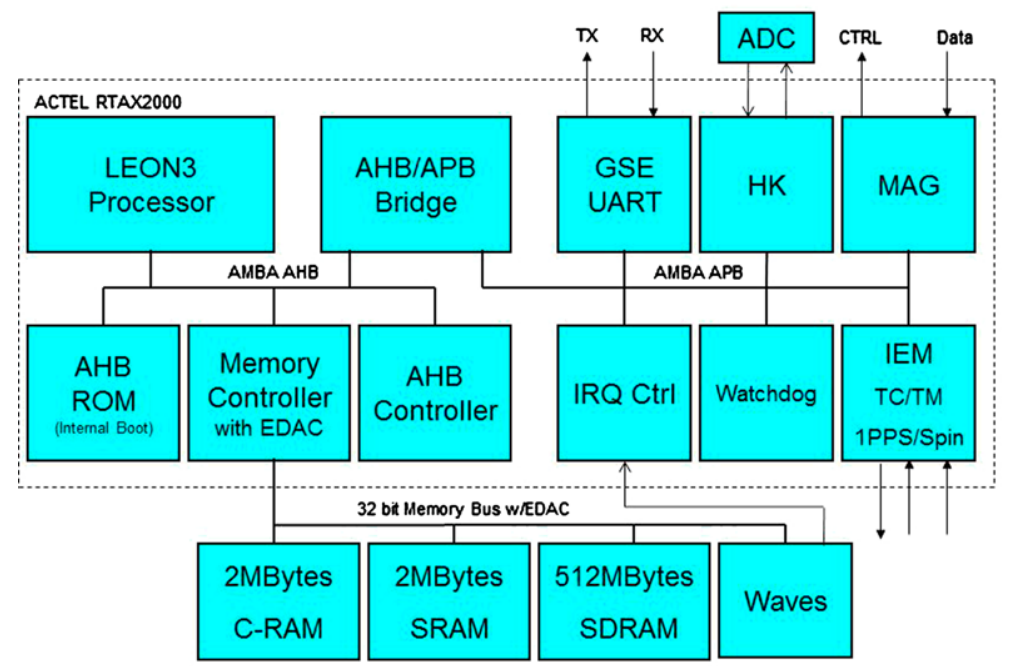

Fig. 21 CDPU block diagram

short burst samples of electric and magnetic field waveforms at higher rates are needed to resolve and identify the detailed physics of wave-particle interactions responsible for radiation belt dynamics, but cannot be transmitted continuously because of telemetry constraints. In addition, spectra are continuously compiled. The Waves FFT boards for both $E$ - and $B$ field analog data rely heavily on field programmable gate arrays to reduce size and power consumption, yet are radiation tolerant, and will supply both survey and burst data.

The CDPU handles compression of the magnetometer data and intermediate storage of all data before transmission to the spacecraft data system. Data from all EMFISIS components are transmitted digitally across the MEB backplane and are stored in a set of buffers which allows the implementation of both 'survey' mode and 'burst' mode data. The CDPU is capable of detecting trigger signals both from EMFISIS instruments and those external to EMFISIS and manages coordination of data modes with the RBSP particle instruments via BURST messages.

\subsection{Heritage}

The CDPU is built upon the LEON-III VHDL core implementation of a SPARC processor. The LEON-III core is programmed into an Actel RTAX2000 FPGA which is radiation hardened to $>100 \mathrm{krad}$, latch-up immune (LET $>100 \mathrm{MeV} \mathrm{cm}^{2} / \mathrm{mg}$ ), and triply-modularredundant for single event upset (SEU) rejection. The basic design of the system, with 512 Mbytes of volatile burst RAM, is a derivative of units on both ISTP and CLUSTER, but in detail is very similar to units being delivered for the NASA MMS program.

\subsection{Functional Description}

Figure 21 shows the architecture of the CDPU board. The area inside the box is logic contained inside the RTAX2000. The LEON processor provides the overall control of the data acquisition from the MAG, WAVES, and Housekeeping interfaces. A Finite State Machine (FSM) in the MAG interface directly controls the timing of the mag vector sampling and 
presents the vector data to the LEON. The interface to the WAVES system is via memory mapped dual ported SRAM on the FFT boards as well as timing signals to initiate FFT Engine acquisitions. Housekeeping data is measured on the LVPS board and multiplexed to the CDPU's Housekeeping ADC. Spacecraft commands and telemetry as handled by the IEM interface module. FSMs receive telecommands from and transmit telemetry to the IEM. Two telecommands are decoded directly by the receive FSM for system reset with vectored booting, watchdog control, and C-RAM (chalcogenide RAM) write protect. All other telecommands are sent to a FIFO and the LEON is interrupted for processing. Telemetry Instrument Transfer Frames (ITFs) are sent by the LEON to a FIFO and a FSM manages the transmission to the IEM.

The CDPU employs four types of memory: (1) AHBROM, (2) C-RAM, (3) SRAM and (4) SDRAM. A Read Only Memory (AHBROM) is programmed internally to the RTAX2000. The AHBROM contains the boot software for the LEON and a simple interrupt handler which can decode simple telecommands. This provides for a robust operation not requiring any of the external memories of the CDPU. If certain faults occur, the CDPU reverts to this robust mode and waits for ground intervention. The other memories are external to the RTAS2000. 2 Mbytes of BAE C-RAM are used for storage of LEON and FFT Engine programs, as well as tables of control information. C-RAM is a new technology which is a replacement for legacy EEPROM devices. C-RAM has a high radiation tolerance ( $>1$ MRAD) and fast write cycles. Fast write timing precludes the software algorithms required for EEPROMs. 2 Mbytes of SRAM are used for temporary storage and program execution scratch space. 512 Mbytes of SDRAM allow for storage of large quantities of high rate burst data and slow transmission to the ground of high value data. All memories utilize EDAC protection from SEU data corruption. The LEON provides a background task to scrub the volatile memories to detect and correct any SEUs which may occur.

\subsubsection{EMFISIS Onboard Data Flow}

Command Processing Every second, the IEM transmits an Instrument Transfer Frame (ITF) to EMFISIS. Upon receipt of an ITF, the CDPU triggers a non-maskable interrupt (NMI). The NMI Interrupt service routine (ISR) transfers the ITF from the command FIFO to a buffer in SRAM and sets a flag to announce its arrival. The Real Time Interrupt (RTI) executes at $64 \mathrm{~Hz}$ and it checks for the ITF arrival flag. If found, it starts the Command task to process the IFT. The Command task parses and executes commands. Time-consuming commands are handled by the Test Task which is started when such a command is executed. Only one "Test" may be executed at a time. Other "Tests" will be flagged as errors.

Telemetry Generation Telemetry is sent from EMFISIS to the IEM via Instrument Transfer Frames (ITF). Each ITF consists of a header and a series of CCSDS data packets. The ITF format is described in the RBSP IEM to EMFISIS ICD and The EMFISIS Users Manual.

Upon receipt of the 1 PPS interrupt, an ITF is generated containing one HK packet and as many other packets as are available in the Survey or Burst buffers. Each ITF is limited to $<4096$ bytes and up to 3 ITFs are sent between 1PPS interrupts. Only the first ITF will contain an HK packet and will toggle the Heartbeat Flag. The $64 \mathrm{~Hz}$ RTI resumes the Packet task. If any data is ready for packetization the packet task collects the data and places the CCSDS packets in the Survey or Burst Buffers as appropriate. The Survey Buffer is usually emptied on every ITF. There may be a need to buffer Survey data briefly if large Burst packets span ITFs but then the buffered Survey data will catch up on the next ITF. The Survey (WAVES + MAG) telemetry allocation is $9 \mathrm{kbps}$. Since each ITF can handle 4096 bytes $(=32 \mathrm{kbps})$ of survey data it will not backlog for long. 
Data Buffering There are currently 3 data buffers implemented:

1. Survey CCSDS packets

2. Burst CCSDS Packets

3. Burst Waveform Event Capture buffer

The Survey buffer is allocated in SRAM. The Burst Buffer and Event Buffer are allocated in SDRAM. These are circular buffers that are not permitted to overrun. If a Survey Packet spans the ITF then no Burst data is sent. Only Survey or Burst data can span an ITF, not both. When an Event has been captured and is selected for download, it is transferred into the Burst Buffer.

Waves Interface The WAVES programs operate synchronous to the $64 \mathrm{~Hz}$ interval and all modes operate on a 6 s cycle divided into 384 intervals. Any WAVES operation can be configured to start on a given phase (0-383) and be repeated after the specified number of intervals. The configuration parameters for a given operation are loaded into a WAVES FFT engine Job Control Block during the interval preceding the desired operation. The FFT engine will execute the configured "program" beginning on the next $64 \mathrm{~Hz}$ pulse. When the program is completed, the FFT engine generates an interrupt to the CDPU. The Job Control Block identifies the type of data, size and locations to be fetched from the FFT Engine and packetized for telemetry or Burst buffering.

\section{EMFISIS Operational Modes}

This section describes the science modes implemented in the CDPU for the EMFISIS investigation. The data consists of two separate types: Magnetometer data and Waves data. The former is simple and constant: 64 vectors per second, in one of three ranges, and are transmitted in losslessly compressed form in all states of instrument operation. The latter is more complex and varied. The various Waves modes span a wide range of telemetry rates, including a basic survey mode which is always running plus a variety of burst modes that provide high time resolution samples. Because the burst modes exceed the average Waves telemetry allocation, these can only be used for limited intervals so that the average Waves telemetry production does not exceed its allocation averaged over a time interval of the order of one week. That is, burst data may be acquired over a relatively short interval of less than an hour, but take as much as a week to be telemetered to the ground. Typically, however, the burst data will be distributed more evenly, with some bursts scheduled each day or perhaps even each orbit. The combination of the Waves FFT engines and EMFISIS CDPU allows for a large range of modes that can be used for various scientific objectives on RBSP and can be updated and changed in flight. The modes listed below represent a reasonable first set and demonstrate the capabilities of the instrument. It is anticipated that variations of these can be defined in-flight by setting various parameters in the CDPU. These Waves modes are summarized in Table 2.

\subsection{Common Features of Waves Science Modes}

Each of the Waves science modes described here have the following common features: 
Table 2 Summary of nominal Waves modes and associated telemetry rates

\begin{tabular}{lcl}
\hline Mode & $\begin{array}{l}\text { Telemetry rate } \\
(\mathrm{kbps})\end{array}$ & Comments \\
\hline Survey & 7.5 & Includes $0.5 \mathrm{~s}$ waveforms every 15 minutes \\
Fast Survey & $\approx 65$ & Includes $0.5 \mathrm{~s}$ waveforms every $30 \mathrm{~s}$ \\
Waveform Burst with Wave Summaries & 1260 & Includes waveforms with $93.6 \%$ duty cycle \\
Waveform Burst with Spectral Matrices & 1265 & Includes waveforms with $93.6 \%$ duty cycle \\
Continuous Waveform Burst & 1300 & Waveforms only with $99.4 \%$ duty cycle \\
30 ms Wave Summaries & 190 & Includes $0.5 \mathrm{~s}$ waveforms every $30 \mathrm{~s}$ \\
$30 \mathrm{~ms}$ Spectral Matrices & 1295 & Includes $0.5 \mathrm{~s}$ waveforms every $30 \mathrm{~s}$ \\
\hline
\end{tabular}

High Frequency Spectrum Each of the science modes includes a high frequency spectrum every $6 \mathrm{~s}$. The high frequency spectrum is derived from the Wave High Frequency Receiver (HFR) and covers the frequency range from 10 to $500 \mathrm{kHz}$ with limited response above $400 \mathrm{kHz}$ due to roll-off of the EFW signals. A 14-bit A/D converter is used to sample the waveform in this passband at a rate of 1.25 million samples per second (1.25 Msps). Blocks of 4096 samples are collected and analyzed on-board using a fast Fourier transform (FFT), yielding a spectrum with frequency spacing of $\approx 305 \mathrm{~Hz}$. Subsequently, the samples are binned into quasi-logarithmically-spaced bins with a spectral resolution of about 50 channels per decade. A programmable number of $N$ waveforms (nominally 6) are analyzed within a $0.5 \mathrm{~s}$ interval and the results are averaged within the spectral bins to increase the signal-tonoise ratio. All processing is carried out in one of the Waves FFT engines. The results are stored in 16-bit truncated floating point values and packaged by the CDPU for intermediate storage in the EMFISIS Mass Memory (MM) for eventual transmission to the RBSP data system and, subsequently, to the ground.

Compressed Waveforms Since each mode includes survey information relying on on-board processing (either spectral matrices or wave property summaries), all modes periodically include 'dual-routed' compressed waveforms. Dual-routed means that the same waveform data used on-board to compute either spectral matrices or wave property summaries are also included in the downlinked telemetry so that the on-board processing can be validated. The waveforms comprise 6 simultaneously-sampled channels ( 3 electric and 3 magnetic), each consisting of $16 \mathrm{k}$ samples with 16-bit resolution over a bandpass of $10 \mathrm{~Hz}$ to $12 \mathrm{kHz}$. The sample rate is 35,000 samples per second. Hence, each set of waveforms covers a $0.468 \mathrm{~s}$ interval. The spectral resolution afforded by the waveforms is, therefore, approximately $2 \mathrm{~Hz}$ if the entire 16-k block is Fourier transformed. Alternately, the blocks can be broken into shorter series (e.g. $1 \mathrm{k}$ blocks) on the ground, allowing greater time resolution for the analysis of, for example, chorus. The waveforms are losslessly compressed by a Rice compression scheme implemented in the Waves FFT engines. The compressed waveforms are delivered to the CDPU for intermediate storage in the EMFISIS mass memory for eventual transmission to the spacecraft and, subsequently, to the ground. For each survey mode, the $0.468 \mathrm{~s}$ waveform captures are performed every nth $6 \mathrm{~s}$ instrument cycle. For survey modes other than the standard survey mode, the nominal value of $n$ is 5 , hence, one waveform data set is returned every $30 \mathrm{~s}$, for a duty cycle of $1.6 \%$. However, for the standard survey modes, $n$ is 150 , hence, one waveform data set is returned every 15 minutes, for a duty cycle of $0.05 \%$. For the Fast Survey mode, $n$ is 5, or a waveform data set every $30 \mathrm{~s}$, giving a duty cycle of $1.6 \%$. The value of $n$ is a variable that can be adjusted on the basis of in-flight experience. 
Low Frequency Wave Information All instrument modes include information on the spectrum and wave mode of waves in the range of $10 \mathrm{~Hz}$ to $12 \mathrm{kHz}$. While raw waveforms allow for the maximum flexibility in ground processing and analysis, the data rate required for these is prohibitive except for a very small percentage of time in any given day. In fact, the Waves telemetry allocation is geared to allow a continuous set of waveform data covering only 30 minutes every three 9 hour orbits (approximately 1 day). Therefore, a more bit-efficient method of transmitting the wave information is required. There are two options afforded by the EMFISIS instrument.

First, spectral matrices can be calculated on-board and the results binned into quasilogarithmically-spaced frequency bins at a resolution of about 20 bins per decade in frequency. For the frequency range $10 \mathrm{~Hz}$ to $12 \mathrm{kHz}$, this results in 61 channels (with 4 channels between 2 and $10 \mathrm{~Hz}$ ). Spectral matrices include auto-correlations of each channel (e.g. $E_{i} E_{i}^{*}$ and $B_{i} B_{i}^{*}$ for $\left.i=U, V, W\right)$ as well as cross-correlations between each pair of sensors resulting in 6 real and 15 complex quantities (since the matrix is symmetric, only half of the off-diagonal terms need be computed; that is, $E_{U} E_{V}^{*}=E_{v} E_{U}^{*}$. Hence, the quantities computed on-board are:

$\begin{array}{llllll}E_{U} E_{U}^{*} & E_{U} E_{V}^{*} & E_{U} E_{W}^{*} & E_{U} B_{U}^{*} & E_{U} B_{V}^{*} & E_{U} B_{W}^{*} \\ E_{V} E_{V}^{*} & E_{V} E_{W}^{*} & E_{V} B_{U}^{*} & E_{V} B_{V}^{*} & E_{V} B_{W}^{*} & \\ E_{W} E_{W}^{*} & E_{W} B_{U}^{*} & E_{W} B_{V}^{*} & E_{W} B_{W}^{*} & & \\ B_{U} B_{U}^{*} & B_{U} B_{V}^{*} & B_{U} B_{W}^{*} & & & \\ B_{V} B_{V}^{*} & B_{V} B_{W}^{*} & & & & \\ B_{W} B_{W}^{*} & & & & & \end{array}$

The quantities in the first column are real and the remaining ones are complex. The real values provide the spectral density in each of the electric and magnetic orthogonal coordinates and can be summed to give the full wave electric and magnetic spectral density as a function of frequency. The off-diagonal terms are used by various algorithms on the ground to determine the wave normal angle, polarization, Poynting flux, etc. Having the spectral matrix on the ground allows for the use of different analysis algorithms. The spectral matrices require a number of computations, including calibration of the signals, despinning of the measurements over the $\approx 0.5 \mathrm{~s}$ collection period (since the spacecraft rotates $\approx 15^{\circ}$ during the collection period), Fourier transforms, the auto- and cross-correlations, and the binning and averaging within spectral bins. All of these are performed in the Waves FFT engines. Each autocorrelation is represented by a 16-bit value and each complex cross-correlation is represented by a 16-bit real value and 16-bit imaginary value. The binned spectral matrices are sent to the CDPU for intermediate storage in the EMFISIS mass memory for eventual transmission to the spacecraft and, subsequently, to the ground.

All modes with spectral matrices in the mode name plus the Survey mode include spectral matrices at least every $6 \mathrm{~s}$ instrument cycle, occurring in the same half-second as the high frequency spectrum. In all survey modes including spectral matrices, the spectral matrices are calculated from 16-k waveform blocks. The 16-k blocks afford reasonable spectral resolution in the lower part of the $10 \mathrm{~Hz}-12 \mathrm{kHz}$ band, but do not allow for high temporal resolution (sub-half-second) that is required for the proper determination of the propagation characteristics of individual chorus elements (although the matrices include valid information on the chorus spectrum averaged over $\approx 0.5 \mathrm{~s}$ ). The temporal and spectral resolution, however, is thought to be adequate for EMIC waves, plasmaspheric hiss, and similar emissions displaying a more slowly varying spectrum.

Second, the spectral matrices can be used as input to further on-board processing to calculate a set of wave property summaries for the waves. The primary benefit of this is that 
the wave property summaries can be telemetered to the ground with significantly fewer bits (of order $90 \%$ less) than the spectral matrices. However there is no way to apply different analysis algorithms to the measurements once these parameters have been calculated onboard as the computations are irreversible. And, it is known that any given algorithm, e.g. the Means method for wave normal analysis, may not work under certain conditions. This is a primary reason why some waveforms are 'dual-routed' in each mode so that the results of the on-board analysis can be validated for a fraction of the measurements.

The wave parameters included in the wave parameter summaries include $E^{2}, B^{2}$, two wave-normal angles, a measure of the planarity of the waves, the ellipticity and polarization sense (Santolik et al. 2003), and the parallel component of the Poynting flux. The spectral matrices are computed in the Waves FFT engine and the CDPU computes the wave property summaries. All modes with wave summaries in the mode name include wave parameter summaries at least every $6 \mathrm{~s}$ instrument cycle, occurring in the same half-second as the high frequency spectrum. In all survey modes including wave summaries, the wave parameters are calculated from 16-k waveform blocks.

\subsection{Descriptions of Waves Science Modes}

The following descriptions give the details of the primary modes of operation of the EMFISIS investigation.

Survey This mode includes a high frequency spectrum and a set of spectral matrices every $6 \mathrm{~s}$. In addition, it includes a set of compressed waveforms every 150 six-second instrument cycles. All three types of data of this mode are acquired during the same half-second interval. The parameter $n$ (nominally set to 150) which sets the repetition rate for the compressed waveforms is a variable to allow variations in the telemetry rate for this mode, depending on in-flight experience with how often the on-board processing needs to be validated. This mode is expected to be the primary survey mode for the mission.

It should be mentioned that an even lower data rate survey mode could be envisioned that substitutes the wave parameter summaries described in Sect. 7.1 for the spectral matrices, however, it would be necessary to gain significant in-flight experience with the spectral matrices to understand how regularly the on-board computation of the wave summaries yield valid results, based on ground simulation of the on-board algorithms. Should this yield be high, and if the conditions under which the on-board algorithms are successful can be reasonably well understood, then a substitution of wave summaries for the spectral matrices would be considered. Such a mode can be implemented on orbit by minor changes to the mode configuration setup.

Fast Survey This mode includes a high frequency spectrum from the HFR receiver every $0.5 \mathrm{~s}$ and a set of spectral matrices from the WFR receivers every second. In addition, its nominal setup includes compressed waveforms every 30 one-second instrument cycles. The rate at which the waveforms are sent is an adjustable parameter, allowing variation from the nominal rate.

Waveform Burst with Wave Summaries This mode includes a high frequency spectrum and a set of wave summaries every $6 \mathrm{~s}$. In addition it includes compressed waveforms every five $6 \mathrm{~s}$ instrument cycles. The high frequency spectrum and wave summaries are acquired during the same half-second interval. In addition, a $0.468 \mathrm{~s}$ set of compressed waveforms is captured every $0.5 \mathrm{~s}$. Hence, every 12 th set of compressed waveforms is obtained simultaneously with 
a set of wave summaries. Another version of the mode replaces the continuous waveforms for $5.968 \mathrm{~s}$ between each $6 \mathrm{~s}$ survey data set. This is referred to as continuous waveform burst mode.

Waveform Burst with Spectral Matrices This mode includes a high frequency spectrum and a set of spectral matrices every $6 \mathrm{~s}$. In addition it includes compressed waveforms every 5 six-second instrument cycles. The high frequency spectrum and spectral matrices are acquired during the same half-second interval. In addition, a 0.468 s set of compressed waveforms is captured every $0.5 \mathrm{~s}$. Hence, every 12 th set of compressed waveforms is obtained simultaneously with a set of spectral matrices.

Continuous Waveform Burst Mode This mode is very similar to the Waveform Burst with Spectral Matrices Mode in that it produces a standard survey set of spectral matrices every based on a $0.5 \mathrm{~s}$ waveform capture every $6 \mathrm{~s}$. In addition. this mode captures a continuous waveforms for $5.968 \mathrm{~s}$ between each $6 \mathrm{~s}$ survey data set, leaving a $0.032 \mathrm{~s}$ gap between successive captures. This mode can be operated continuously for fixed durations up to several minutes. Alternatively it can be used in conjunction with burst quality flags that retain the best $N 5.968$ s captures $(N \leq 20)$ in a pre-set time interval.

$30 \mathrm{~ms}$ Wave Summaries This mode is based on the survey with wave summaries mode in that it includes a high frequency spectrum and a set of wave summaries every $6 \mathrm{~s}$. In addition it includes compressed waveforms every 5 six-second instrument cycles. All of the data types in this mode are acquired during the same half-second interval. The parameter $\mathrm{n}$ (currently set to 5) which sets the repetition rate for compressed waveforms will be a variable to allow variations in the telemetry rate for this mode, depending on in-flight experience with how often the on-board processing needs to be validated. In addition to the survey data, the mode includes high rate wave summaries at a rate of 1 set per $30 \mathrm{~ms}$. The wave summaries are based on $1 \mathrm{k}$ blocks (30 $\mathrm{ms}$ ) of waveform data and the frequency bins are linear, with spacing of about $150 \mathrm{~Hz}$. The purpose of this mode is to provide high temporal resolution information on the propagation characteristics of chorus, whose individual elements have time scales of order $30 \mathrm{~ms}$. The processing includes tests of planarity and coherency, hence, properties other than $E^{2}$ and $B^{2}$ need not be returned if these tests fail, reducing the required telemetry. The rates estimated for this mode assume that $75 \%$ of the frequency-time bins fail these tests, hence, only the spectral densities are returned for those bins. With verification that the on-board algorithms are reliable, this mode will provide propagation information on discrete chorus structures over a much longer time interval than afforded by the very high telemetry rates of the waveform burst modes.

$30 \mathrm{~ms}$ Spectral Matrices This mode is based on the survey mode in that includes a high frequency spectrum and a set of spectral matrices every $6 \mathrm{~s}$. In addition it includes compressed waveforms every 5 six-second instrument cycles. All of the data types in this mode are acquired during the same half-second interval. The parameter $n$ (currently set to 5) which sets the repetition rate for compressed waveforms will be a variable to allow variations in the telemetry rate for this mode, depending on in-flight experience with how often the on-board processing needs to be validated. In addition to the survey data, the mode includes high rate spectral matrices at a rate of 1 set per $30 \mathrm{~ms}$. The spectral matrices are based on $1 \mathrm{k}$ blocks $(30 \mathrm{~ms}$ ) of waveform data and the frequency bins are linear, with spacing of about $150 \mathrm{~Hz}$.

The purpose of this mode is to provide high temporal resolution information on the propagation characteristics of chorus, whose individual elements have time scales of order $30 \mathrm{~ms}$. 
Chorus is typically found in the frequency range of 0.1 to $0.7 f_{c e}\left(f_{c e}=\right.$ electron cyclotron frequency where $\left.f_{c e}[\mathrm{~Hz}]=28|B|[\mathrm{nT}]\right)$. Hence, the telemetry may be reduced by returning only those frequency bins in this frequency range. $|B|$ is a function of radial distance, among other things, therefore, these data volume savings are also a function of position. The telemetry rates assume a worst case spectral range of 1.6 to $12 \mathrm{kHz}$, or, 69 frequency bins. Since the spectral matrices are more efficient in telemetry than the waveform burst modes, this mode can be used to extend the time interval over which the propagation characteristics of discrete chorus elements are determined. The spectral matrices allow for various types of analysis on the ground that are not afforded by the high time resolution wave summaries in the $30 \mathrm{~ms}$ Wave Summaries mode and may allow recovery of wave propagation information where the on-board algorithm fails.

\section{EMFISIS Data: Reduction, Products, Distribution, and Archiving}

\subsection{RBSP EMFISIS/MAG Instrument Data Processing}

Telemetry allocations permitted the early adoption of a simple operation model for the RBSP EMFISIS/MAG instrument. Specifically, there is only one instrument mode so that the instrument functions in the same manner collecting data at the same rate at all times. However, there are four different data forms (channels) that are dictated by the needs of the EMFISIS central processor and by the needs of the spacecraft to obtain usable MAG data in the event that the central processor should fail. Each data channel is assigned a unique identifier to ensure proper processing. EMFISIS MAG data is processed in a day-by-day manner without reference to data from prior or subsequent days.

Data Description The EMFISIS/MAG instrument makes 64 vector/s measurements at all times in the unrectified (nonorthogonal) coordinate system known as "MAG sensor" MAG maintains its own internal clock to divide the spacecraft $1 \mathrm{~s}$ clock into 64 equal intervals.

MAG Data Reconstruction Although the MAG instrument should run continuously, there may be data gaps in telemetry. The first step in data processing is to reconstruct the true spacecraft clock time as a combination of the spacecraft MET time and the MAG clock time. The second step is to identify data gaps and fill them with data flagged as bad or missing. This adds a processing flag (ifillflag) that marks $1 \mathrm{~s}$ packets of missing or fill data. In the process, we check for possible data packets containing time tags but no usable data and data out of range for any reason, both of which are flagged as fill. Compressed data are decompressed. Failsafe data, packets are completed with out-of-range fill values to the extent they are incomplete packets. EMFISIS/MAG data are processed one packet at a time. Any merger of packet types is performed as a post-process only. This first step in data processing is designed to provide the end user with guaranteed contiguous data products.

Application of Calibration and Sensor Axis Alignment The three internal axis sensors are not perfectly rectified or aligned with the nominal UVW axis directions. Two rotation matrices are measured in the lab and applied to the measurements in flight:

$$
B_{\text {sensor }}=A_{1} G R_{\text {ortho }}(C-Z)
$$

where $C$ is a three-element vector of the 16-bit raw data counts, $Z$ is a three-element vector applied to the counts to remove any offset relative to 0 (range dependent), $R_{\text {ortho }}$ is a 
$3 \times 3$ matrix to create an orthogonal coordinate system from the three internal sensor directions (independent of range), $G$ is a three-element vector that converts zero-corrected counts to $\mathrm{nT}$ (range dependent), and $A_{1}$ is a rigid rotation matrix to rotate the sensor coordinate system to the nominal $(U V W)$ science payload coordinate system assuming perfect magnetometer boom alignment. However, it is not perfectly aligned, and therefore an additional rigid rotation matrix $A_{2}$ is required to complete rotation of the measured field to $(U, V, W)$ coordinates:

$$
B_{U V W}=A_{2} B_{\text {sensor }} .
$$

All of these matrices are pre-determined in the laboratory before flight. Only the product of these can be determined in flight.

Coordinate Rotation Once the MAG data is obtained in physical units in the $(U, V, W)$ science payload coordinate system it is a simple matter to rotate to other useful coordinate systems. The first of these is the spacecraft payload system $(X, Y, Z)$ which is a 35 degree rotation from $(U, V, W)$. This is useful mainly for diagnostic purposes and will not be made available to the general public unless requested. Science coordinate systems include GSE, GSM, SM, GEI and GEO.

Payload Coordinate Data Products In order to facilitate any magnetic field needs by other instruments on the spacecraft, EMFISIS/MAG will provide data at full 64 vector/sec resolution in $(U, V, W)$ science payload coordinates.

Science Data Products EMFISIS/MAG plans to provide a broad range of data products providing ambient magnetic field data in a variety of coordinate systems and time resolutions. Time resolutions are the full 64 vectors/sec as well as $4 \mathrm{~s}$ averages where averaging intervals are aligned with packet boundaries. Daily graphs of the science data are planned using IDL in a fully automated form. These will include spacecraft location and magnetic field predictions for one or more storm time expectations as an aid in assessing the data graphed. Multi-panel spectrograms will also be created including the power, polarization, and ellipticity of the magnetic fluctuations.

\subsubsection{MAG Data Example}

Figure 22 shows an example of MAG data from October 31, 2013 when the RBSP-A spacecraft was near apogee. The data are in the GSM coordinate system and present an example of a sudden depolarization of the Earth's magnetic field as indicated by rapid increase in the magnitude of the $z$ component around 15:40 UT. This rapid field change is followed by low frequency wave signatures which suggest both ULF and EMIC waves were driven as a result.

\subsection{Waves Data Reduction and Products}

The Waves data products will be released as a set of files in Common Data Format (CDF) format. The data will be time-ordered and non-duplicating, and the Level 1 and 2 products will be limited to a single data product per file. The following list describes each of the Waves data products, and the types of primary data that will be found in each.

1. $30 \mathrm{~ms}$ Spectral Matrices (APID 0x2b5)—component spectrograms, 70 frequencies sampled at $35 \mathrm{kHz}$. 


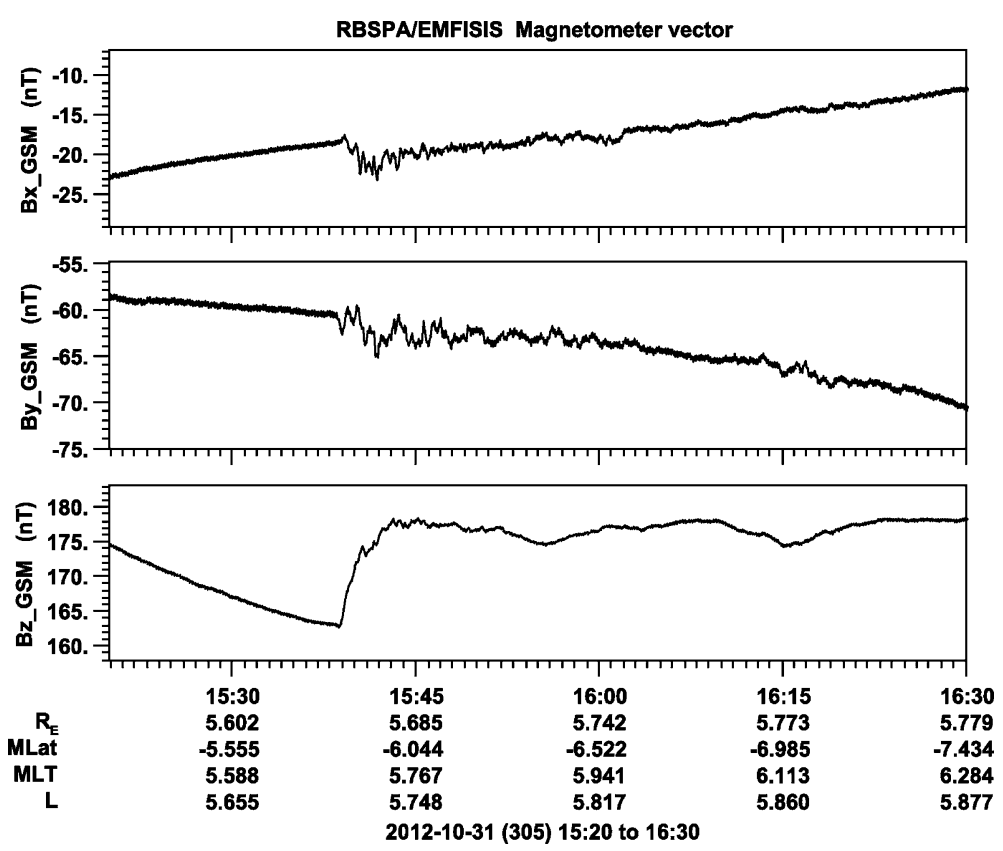

Fig. 22 Example of MAG data from the RBSP-A spacecraft on October 31, 2012 in GSM coordinates

- $B_{U} B_{U}, B_{V} B_{V}, B_{W} B_{W}$-Autocorrelation of this component, $\mathrm{nT}^{2} / \mathrm{Hz}$

- $E_{U} E_{U}, E_{V} E_{V}, E_{W} E_{W}$-Autocorrelation of this component, $(\mathrm{V} / \mathrm{m})^{2} / \mathrm{Hz}$

- $B_{U} B_{V}, B_{U} B_{W}, B_{V} B_{W}$ - Cross multiply of components, $\mathrm{nT}^{2} / \mathrm{Hz}$ (complex)

- $E_{U} E_{V}, E_{U} E_{W}, E_{V} E_{W}$ - Cross multiply of components, (V/m) $)^{2} / \mathrm{Hz}$ (complex)

- $B_{U} E_{U}, B_{U} E_{V}, B_{U} E_{W}, B_{V} E_{U}, B_{V} E_{W}, B_{V} E_{W}, B_{W} E_{U}, B_{W} E_{V}, B_{W} E_{W}$-Cross multiply of components, $\mathrm{nT}(\mathrm{V} / \mathrm{m}) / \mathrm{Hz}$ (complex)

2. WFR Spectral Matrices (APID 0x2b2)—spectrograms, 65 frequencies

- Same as 30 ms mode.

3. WFR Spectral Matrices, burst-mode (APID 0x2b3)—-spectrograms, 65 frequencies

- Same as $30 \mathrm{~ms}$ mode.

4. HFR Spectra (APID 0x2a1)—spectrograms, 82 frequencies

- HFR_Spectra-Autocorrelation single E component, $(\mathrm{V} / \mathrm{m})^{2} / \mathrm{Hz}$

5. HFR Spectra Burst (APID 0x2a3)—spectrograms, 82 frequencies

- HFR_Spectra-Autocorrelation single E component, $(\mathrm{V} / \mathrm{m})^{2} / \mathrm{Hz}$

6. HFR Waveforms (APID 0x2a0)

- HFR Samples-Electric field, V/m

7. WFR Waveform (APID 0x2b0)

- $B_{U}, B_{V}, B_{W}$-Magnetic field, nT

- $E_{U}, E_{V}, E_{W}$-Electric field, V/m 
8. WFR Waveform Burst (APID 0x2b4)

- $B_{U}, B_{V}, B_{W}$-Magnetic field, nT

- $E_{U}, E_{V}, E_{W}$-Electric field, V/m

9. WFR Waveform Continuous Burst (APID 0x2b8)

- $B_{U}, B_{V}, B_{W}$-Magnetic field, nT

- $E_{U}, E_{V}, E_{W}$-Electric field, $\mathrm{V} / \mathrm{m}$

10. Wave Normal Analysis (APID 0x2b6)

$-B^{2}$-Squared magnetic field magnitude, $\mathrm{nT}^{2} / \mathrm{Hz}$

- $E^{2}$-Squared electric field magnitude, $(\mathrm{V} / \mathrm{m})^{2} / \mathrm{Hz}$

- $S_{x}$-Poynting Vector, $\mathrm{W} / \mathrm{m}^{2}$

- $S_{y}$-Poynting Vector, $\mathrm{W} / \mathrm{m}^{2}$

- $S_{z}$-Poynting Vector, $\mathrm{W} / \mathrm{m}^{2}$

- Magnetic eigenvectors and eigenvalues - arbitrary units with component values for vectors.

In addition, each data L0 product contains the following meta-data:

- LWEzGainW-0 = normal, $1=$ attenuated $(19 \mathrm{~dB})$

- LWExEyGainUV-0 = normal, $1=$ attenuated $(19 \mathrm{~dB})$

- HBGain- $0=$ normal, $1=$ attenuated $(19 \mathrm{~dB})$

- HBSelect $-0=U, 1=V, 2=W, 3=$ unused

- SCMBW-0 = normal, $1=$ limited

- SCMGain-0 = normal, $1=$ attenuated $(19 \mathrm{~dB})$

- ADCPWR- $0=$ on, $1=$ off

- waveformChannel-0 $=$ WFR $B_{X}(U), 1=$ WFR $B_{Y}(V), 2=$ WFR $B_{Z}(W), 3=$ WFR $E_{X}(U), 4=$ WFR $E_{Y}(V), 5=$ WFR $E_{Z}(W), 6=$ HFR-Channel selected by HBSelect, 7 = N/A

- sunPulseTimeTag-Bits 21:16= seconds ( 6 lsb of MET), Bits 15:0= subseconds $(50 \mathrm{mi}-$ croseconds/bit)

- MET-Mission Elapsed Time

QL files, and higher level files such L2 and L3 files, contain subsets of these meta-data as appropriate for the data contained in the file. For example, WFR attenuator data are not included in higher level HFR files.

\subsubsection{Waves Data Examples}

Figure 23 shows an example of the three types of survey mode data returned by the EMFISIS Waves instrument on Van Allen Probe B on November 17-18, 2013. The top panel shows the HFR spectral data from 10-400 kHz. Clearly evident is the upper hybrid line which drops rapidly at the plasmapause due to rapidly decreasing plasma density. Several other wave modes are also present during this active time. The middle and bottom panels show the WFR electric and magnetic spectral data for $10 \mathrm{~Hz}$ to $12 \mathrm{kHz}$. The narrow feature above $700 \mathrm{~Hz}$ is whistler mode chorus and the lower frequency feature seen within the plasmasphere (before 20:00 and after 01:50) is plasmaspheric hiss.

Figure 24 shows an example of a six-channel waveform capture which has been Fourier transformed into spectrogram form. The data are from a snapshot near apogee when the spacecraft was south of the magnetic equator and located slightly before local dawn. The top 

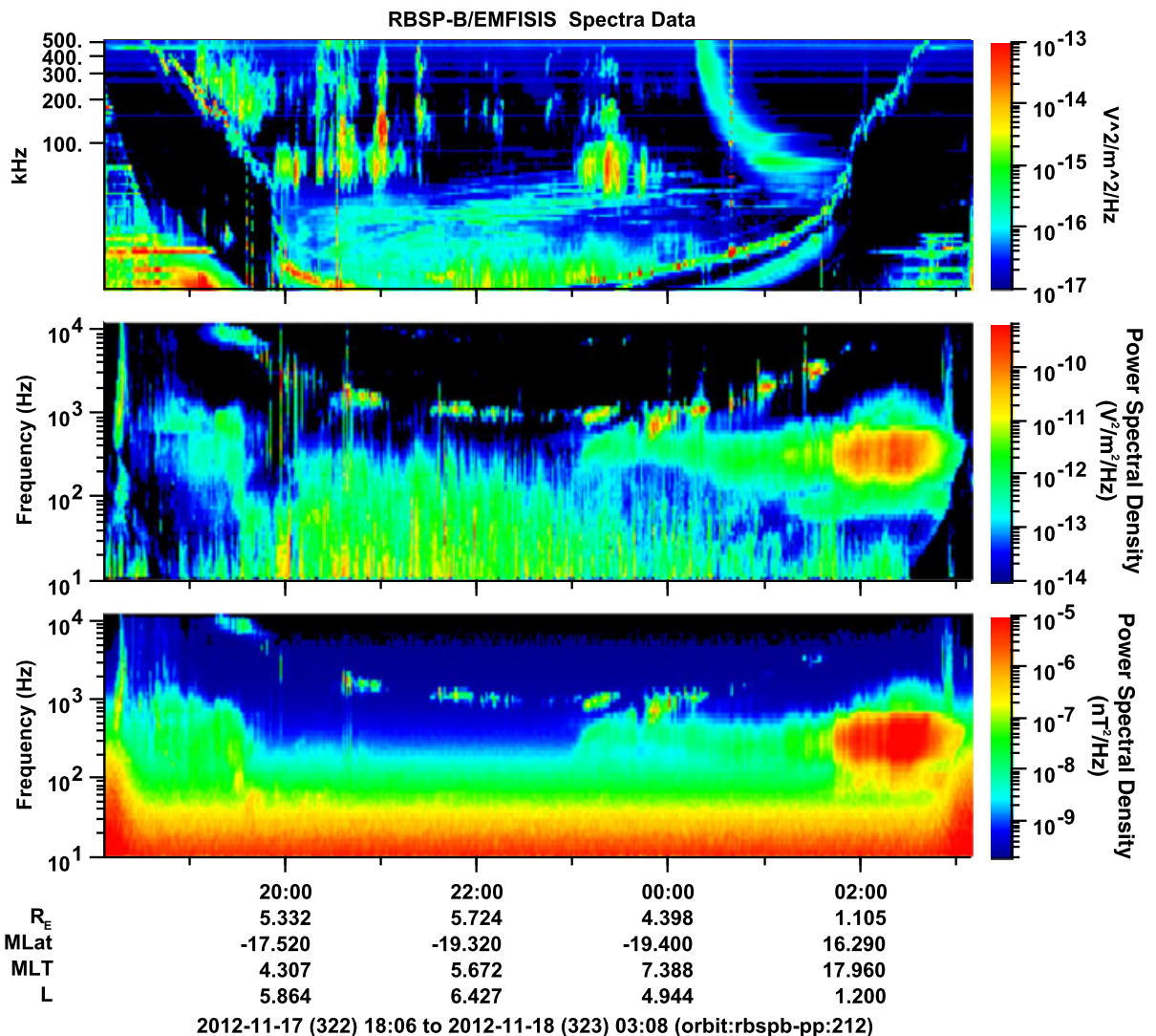

Fig. 23 Example of Waves survey data from November 17-18, 2012. The top panel is the HFR data from $10 \mathrm{kHz}$ to $400 \mathrm{kHz}$, the middle panel is the electric field WFR data from $10 \mathrm{~Hz}$ to $12 \mathrm{kHz}$, and the bottom panel is magnetic field WFR data from $10 \mathrm{~Hz}$ to $12 \mathrm{kHz}$

three panels show the three components of the magnetic field in spacecraft science coordinates, and the lower three panels show the electric field in spacecraft science coordinates. This high time resolution data reveals whistler mode chorus elements with structure of the order of tens of ms.

Figure 25 shows an example of the derived wave parameters for the same data shown in Fig. 24 The top panel shows the total magnetic field measured by the magnetic search coil and calculated using the sum of the squares of the diagonal elements of the spectral matrix calculated from the waveforms. The middle panel shows the wave-normal vector ( $k$-vector) calculated from the spectral full matrix including both $\mathrm{E}$ and $\mathrm{B}$. As can be seen, the wave normal direction is typically less than 20-30 degrees for the chorus elements, but there are some spectral components which have wave normal vectors of up to 60 degrees. The bottom panel of Fig. 25, shows the Poynting flux which is predominantly field-aligned at 180 degrees. This corresponds to propagation in the poleward direction, that is, southward away from the equator. 


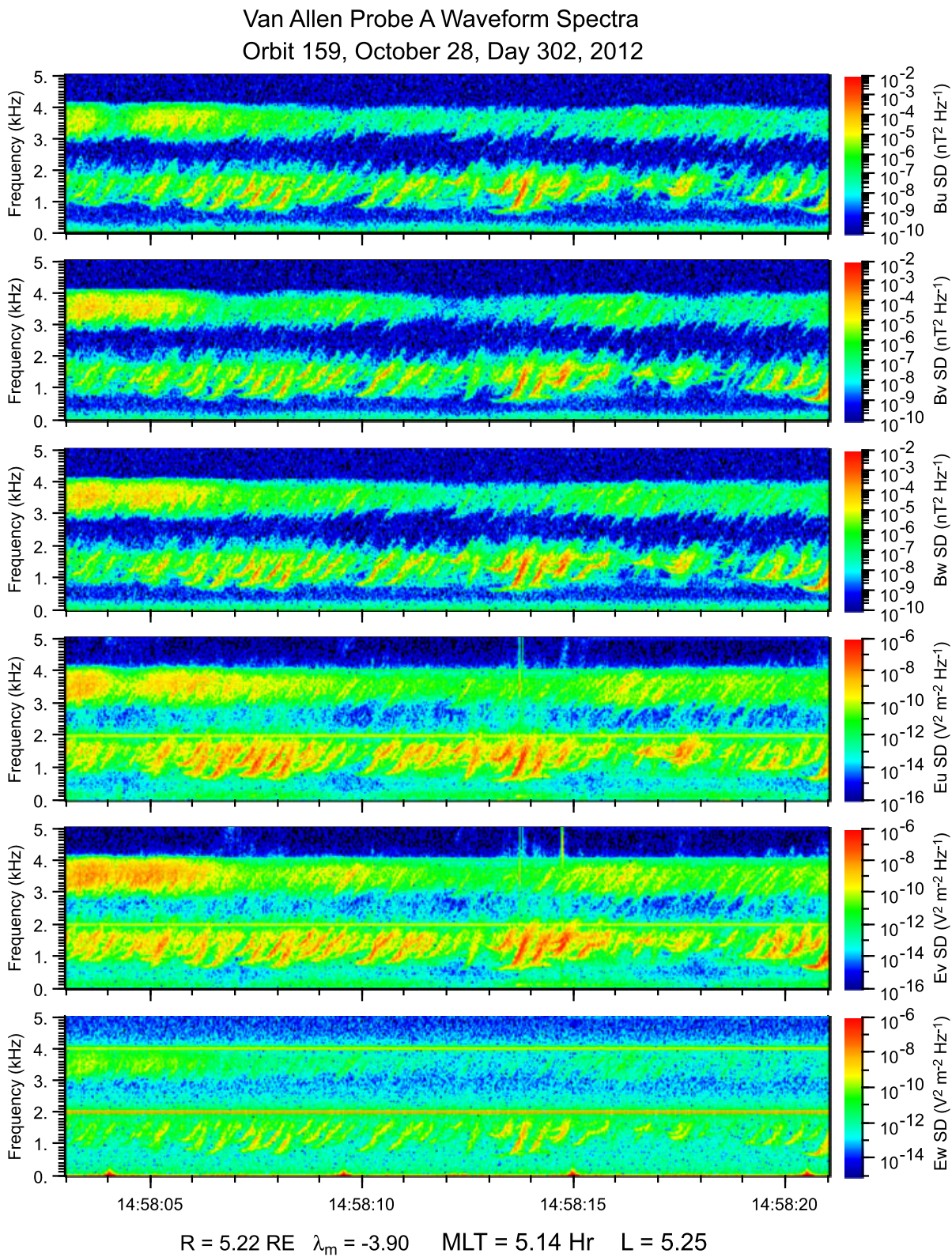

Fig. 24 Example of six-channel waveform data that has been Fourier transformed to produced high time resolution spectrograms

\subsection{Data Distribution}

Level 0 (L0) data products obtained from the Mission Operations Center (MOC) and baselevel calibrated Level 1 (L1) data products created by EMFISIS software at the University of Iowa (see Sect. 8.4, below) are made available to the EMFISIS team members, primarily 

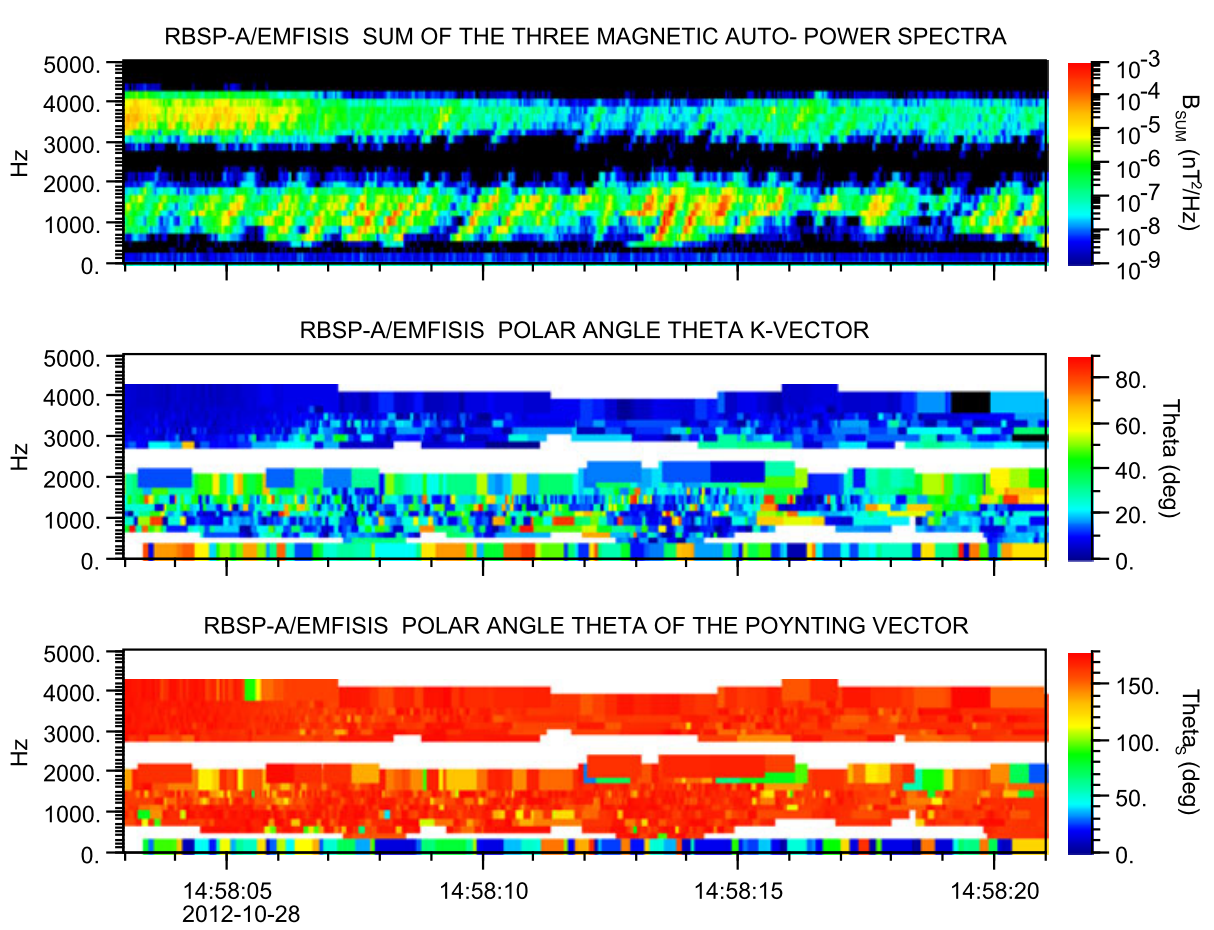

Fig. 25 Wave parameters showing the wave normal direction (middle panel) and Poynting vector (bottom panel) for the same time interval of the six-channel waveform capture shown in Fig. 24

for the purpose of creating higher-level scientific products, and secondarily for analysis and testing of the EMFISIS instrument and its subsystems.

L0 magnetometer products are sent to Dr. Charles Smith at the University of New Hampshire (UNH) for conversion into quick-look data products and higher-level L2, L3, and L4 products. As part of this process, an internal L1 product is created which remains at UNH. These products are then pulled back to the Science Analysis Science Operations Center (SASOC) at the University of Iowa, and made available to team members and the public on the EMFISIS website.

L0 Waves instrument products are converted from raw PTP products to time-tagged uncalibrated L1 Common Data Format (CDF) data products at the University of Iowa (UI). Calibrations are applied to L1 CDFs at UI to further process them into scientific Level 2 (L2) CDFs. All L2 and higher level data products will be made available to the public on the schedule shown in Table 3.

The primary method of access to the EMFISIS data will be the SASOC web server located at http://emfisis.physics.uiowa.edu/. All data products at those levels will be in the ISTP-compliant Common Data Format (CDF) format, details for which can be found at the NASA Space Physics Data Facility web site.

\subsection{Archiving}

In addition to storing EMFISIS products indefinitely at the SASOC website listed in Sect. 8.3, all EMFISIS products L2 and higher will be available through the NASA SPDF 
Table 3 EMFISIS data definitions

\begin{tabular}{|c|c|c|c|c|c|c|}
\hline $\begin{array}{l}\text { Data } \\
\text { level }\end{array}$ & Product title & Contents & Volume & Format & Latency & Frequency \\
\hline L0 & Raw telemetry & $\begin{array}{l}\text { Raw EMFISIS } \\
\text { data including } \\
\text { both science and } \\
\text { HK data }\end{array}$ & $\begin{array}{l}500 \text { Mbytes } \\
\text { per day per } \\
\text { spacecraft }\end{array}$ & $\begin{array}{l}\text { Binary/ISTP } \\
\text { Compliant } \\
\text { CDF }\end{array}$ & $\begin{array}{l}\text { Minutes from } \\
\text { availability at } \\
\operatorname{MOC}\left(T_{0}\right)\end{array}$ & Daily \\
\hline L1 & $\begin{array}{l}\text { Time series and } \\
\text { spectra; Burst } \\
\text { data, Time series } \\
\text { and spectra } \\
\text { (relative } \\
\text { amplitudes); } \\
\text { Burst data }\end{array}$ & $\begin{array}{l}\text { Calibrated DC } \\
\text { Magnetic Field } \\
\text { values (calibrated } \\
\text { and corrected } \\
\text { physical units) }\end{array}$ & $\begin{array}{l}750 \text { Mbytes } \\
\text { per day per } \\
\text { spacecraft }\end{array}$ & $\begin{array}{l}\text { ISTP } \\
\text { Compliant } \\
\text { CDF }\end{array}$ & $T_{0}+<8$ days & Daily \\
\hline L2 & $\begin{array}{l}\text { Calibrated time } \\
\text { series, spectra } \\
\text { and burst data }\end{array}$ & $\begin{array}{l}\text { Spectral } \\
\text { quantities } \\
\text { (calibrated and } \\
\text { corrected } \\
\text { physical units); } \\
\text { Includes low } \\
\text { frequency spectra } \\
\text { from MAG }\end{array}$ & $\begin{array}{l}850 \text { Mbytes } \\
\text { per day per } \\
\text { spacecraft }\end{array}$ & $\begin{array}{l}\text { ISTP } \\
\text { Compliant } \\
\text { CDF }\end{array}$ & $\begin{array}{l}T_{0}+<60 \\
\text { days }(14 \text { days } \\
\text { for MAG) }\end{array}$ & Daily \\
\hline L3 & $\begin{array}{l}\text { Lower level } \\
\text { derived products }\end{array}$ & $\begin{array}{l}\text { Magnetic wave } \\
\text { parameters }\end{array}$ & $\begin{array}{l}850 \text { Mbytes } \\
\text { per day per } \\
\text { spacecraft }\end{array}$ & $\begin{array}{l}\text { ISTP } \\
\text { Compliant } \\
\text { CDF }\end{array}$ & $\begin{array}{l}T_{0}+<4 \\
\text { months }\end{array}$ & Daily \\
\hline L4 & $\begin{array}{l}\text { Higher level } \\
\text { derived products }\end{array}$ & $\begin{array}{l}\text { Wave } \\
\text { propagation } \\
\text { parameters } \\
\text { (spectral } \\
\text { matrices, WNA, } \\
\text { polarization, } \\
\text { Poynting flux, } \\
\text { etc.); Electron } \\
\text { densities }\end{array}$ & $\begin{array}{l}1250 \\
\text { Mbytes per } \\
\text { day per } \\
\text { spacecraft }\end{array}$ & $\begin{array}{l}\text { ISTP } \\
\text { Compliant } \\
\text { CDF }\end{array}$ & $T_{0}+<1$ year & Daily \\
\hline
\end{tabular}

data archive (http://spdf.gsfc.nasa.gov/). Long-term archival through either optical or hard drive media will be evaluated on a yearly basis once RBSP is on-orbit.

\subsection{EMFISIS Data Tools}

\subsubsection{Data-Processing Tools}

All EMFISIS data processing code will be made available at the EMFISIS SOC web site (http://emfisis.physics.uiowa.edu) for examination by the public. These programs are used to convert data from low-level telemetry into functional data formats, to apply calibrations, and to produce higher-level derived products.

The primary EMFISIS software library is a collection of Java classes that unpack the PTP telemetry formatted files from the MOC, further decode the CCSDS packets contained therein, and write their EMFISIS data payloads in CDF format. Additionally, the L0 files use Mission Elapsed Time (MET) from the spacecraft, which is converted using SPICE kernels into UTC time. The L1 data products, with the exception of magnetometer data, are in uncalibrated abstract units. L1 to L2 data file conversion primarily applies calibrations 
and produces data products in physical units. Additionally, data files containing both survey and burst data may be combined into a single additional data file for ease of data analysis. These programs may be written in a variety of languages, such as Java, Python, and IDL.

Higher level data products will be produced by the schedule listed in Table 3, and the source code for those programs will be made available as they are written.

\subsubsection{On-line Tools}

A variety of web-based tools will be hosted at the EMFISIS web site (http://emfisis.physics. uiowa.edu), with a focus on tools that provide meta-data about the products, and tools that aid in organizing the data for the user.

The primary data analysis tool for EMFISIS is Autoplot, a free cross-platform data visualization tool. Autoplot can natively read the CDF format, and convert it into a variety of other formats. Autoplot is scriptable, and is capable of working with data sets from a variety of complementary data tools, such as IDL, Matlab, and the THEMIS Data Analysis Software (TDAS).

\section{Science Closure and Conclusions}

The EMFISIS investigation provides the key set of wave and DC magnetic field measurements needed for significant progress on understanding the detailed physical processes which underlie radiation belt acceleration, loss, and transport as well as the important role that the ring current plays in the inner magnetosphere. As we have demonstrated, EMFISIS has the correct frequency ranges and time resolutions to address the key science questions to be addressed by the RBSP mission. By combining a state-of-the art set of sensors with powerful and flexible central processor and co-processor control, EMFISIS has the needed capability to further our understanding of the Earth's radiation belts.

Acknowledgements We would like to thank the entire Van Allen Probes team who have made this mission the success that it is. We would also like to thank the EFW team, in particular, for their work in supplying electric field signals to EMFISIS as well as supporting cross-calibration of the two sets of instruments. This work was performed under supported on JHU/APL contract no. 921647 under NASA Prime contract No. NAS5-01072.

Open Access This article is distributed under the terms of the Creative Commons Attribution License which permits any use, distribution, and reproduction in any medium, provided the original author(s) and the source are credited.

\section{Appendix A: EMFISIS Key Specifications}

A.1 Magnetometer

Cadence: 64 vectors/s

\begin{tabular}{lllll}
\hline Range ID & Field range & Resolution & $\begin{array}{l}\text { Accuracy } \\
\text { (sensor only) }\end{array}$ & $\begin{array}{l}\text { Accuracy } \\
\text { (deployed on spacecraft) }\end{array}$ \\
\hline 3 & $\pm 65536 \mathrm{nT}$ & $2 \mathrm{nT}$ & $0.1 \mathrm{nT}$ & $5 \mathrm{nT}$ \\
1 & $\pm 4096 \mathrm{nT}$ & $0.16 \mathrm{nT}$ & $0.1 \mathrm{nT}$ & $5 \mathrm{nT}$ \\
0 & $\pm 256 \mathrm{nT}$ & $0.001 \mathrm{nT}$ & $0.1 \mathrm{nT}$ & $5 \mathrm{nT}$ \\
\hline
\end{tabular}




\section{A.2 Waves}

\section{A.2.1 WFR Receiver Performance}

MSC Noise Floor best estimate from lab calibrations:

(Noise levels below $100 \mathrm{~Hz}$ are difficult to ascertain due to $60 \mathrm{~Hz}$ interference)

\begin{tabular}{ll}
\hline Frequency & Noise level \\
\hline $100 \mathrm{~Hz}$ & $<10^{-8} \mathrm{nT}^{2} / \mathrm{Hz}$ \\
$1 \mathrm{kHz}$ & $<10^{-10} \mathrm{nT}^{2} / \mathrm{Hz}$ \\
$10 \mathrm{kHz}$ & $<5 \times 10^{-10} \mathrm{nT}^{2} / \mathrm{Hz}$ \\
\hline
\end{tabular}

Dynamic Range: 96 dB (19 dB switchable attenuator)

Maximum amplitude:

no attenuator: $2.1 \mathrm{nT}$

with attenuator: $9.0 \mathrm{nT}$ (signal begins to distort)

Electric field Noise Floor (100 meter baseline) best estimate from lab calibrations:

\begin{tabular}{ll}
\hline Frequency & Noise level \\
\hline $2.5 \mathrm{~Hz}$ & $<10^{-14}(\mathrm{~V} / \mathrm{m})^{2} / \mathrm{Hz}$ \\
$10 \mathrm{~Hz}$ & $<10^{-15}(\mathrm{~V} / \mathrm{m})^{2} / \mathrm{Hz}$ \\
$100 \mathrm{~Hz}$ & $<5 \times 10^{-15}(\mathrm{~V} / \mathrm{m})^{2} / \mathrm{Hz}$ \\
$1 \mathrm{kHz}$ & $<5 \times 10^{-16}(\mathrm{~V} / \mathrm{m})^{2} / \mathrm{Hz}$ \\
$10 \mathrm{kHz}$ & $<5 \times 10^{-16}(\mathrm{~V} / \mathrm{m})^{2} / \mathrm{Hz}$ \\
\hline
\end{tabular}

Maximum amplitude:

no attenuator: $5.2 \mathrm{mV} / \mathrm{m}$ (spin-plane), $37 \mathrm{mV}$ (axial)

with attenuator: $46 \mathrm{mV} / \mathrm{m}$ (spin-plane), $330 \mathrm{mV} / \mathrm{m}$ (axial)

\section{A.2.2 HFR Receiver Performance}

Noise Floor (100 meter baseline) best estimate from lab calibrations:

\begin{tabular}{ll}
\hline Frequency & Noise level \\
\hline $10 \mathrm{kHz}$ & $<10^{-17}(\mathrm{~V} / \mathrm{m})^{2} / \mathrm{Hz}$ \\
$100 \mathrm{kHz}$ & $<10^{-17}(\mathrm{~V} / \mathrm{m})^{2} / \mathrm{Hz}$ \\
$500 \mathrm{kHz}$ & $<2 \times 10^{-17}(\mathrm{~V} / \mathrm{m})^{2} / \mathrm{Hz}$ \\
\hline
\end{tabular}

Dynamic Range: 84 dB (19 dB switchable attenuator)

Maximum amplitude:

no attenuator: $1.4 \mathrm{mV} / \mathrm{m}$ (spin-plane), $10 \mathrm{mV}$ (axial)

with attenuator: $14 \mathrm{mV} / \mathrm{m}$ (spin-plane), $100 \mathrm{mV} / \mathrm{m}$ (axial)

\section{A.3 Standard Survey Products}

Note that in what follows the names used are those which correspond to the data products rather than the names of the modes described above. There is a close correspondence, but for data users, the names below match those of files containing the data that is described. 


\section{A.3.1 HFR-Spectra}

$E_{U}$ or $E_{V}$ or $E_{W}$ :

Cadence: 6 s 4096 samples at 1 MSamples per second (14 bit digitization)

Bin averaged into 82 logarithmically spaced bins between $10 \mathrm{kHz}$ to $500 \mathrm{kHz}$

\section{A.3.2 WFR-Spectral-Matrix}

Three Axis Electric Field $\left(E_{U}, E_{V}, E_{W}\right)$ and Three Axis Magnetic field $\left(B_{U}, B_{V}, B_{W}\right)$ cross spectral matrix, 6 diagonal components and 15 off-diagonal components:

\begin{tabular}{l|llllll} 
& $E_{U}$ & $E_{V}$ & $E_{W}$ & $B_{U}$ & $B_{V}$ & $\mathbf{B}_{W}$ \\
\hline$E_{U}$ & $E_{U} E_{U}$ & $E_{U} E_{V}$ & $E_{U} E_{W}$ & - & - & - \\
$E_{V}$ & - & $E_{V} E_{V}$ & $E_{V} E_{W}$ & - & - & - \\
$E_{W}$ & - & - & $E_{W} E_{W}$ & - & - & - \\
$B_{U}$ & $B_{U} E_{U}$ & $B_{U} E_{V}$ & $B_{U} E_{W}$ & $B_{U} B_{U}$ & $B_{U} B_{V}$ & $B_{U} B_{W}$ \\
$B_{V}$ & $B_{V} E_{U}$ & $B_{V} E_{V}$ & $B_{V} E_{W}$ & - & $B_{V} B_{V}$ & $B_{V} B_{W}$ \\
$B_{W}$ & $B_{W} E_{U}$ & $B_{W} E_{V}$ & $B_{W} E_{W}$ & - & - & $B_{W} B_{W}$
\end{tabular}

Units:

\begin{tabular}{l|llllll} 
& $E_{U}$ & $E_{V}$ & $E_{W}$ & $B_{U}$ & $B_{V}$ & $\mathbf{B}_{W}$ \\
\hline$E_{U}$ & $(\mathrm{~V} / \mathrm{m})^{2} / \mathrm{Hz}$ & $(\mathrm{V} / \mathrm{m})^{2} / \mathrm{Hz}$ & $(\mathrm{V} / \mathrm{m})^{2} / \mathrm{Hz}$ & - & - & - \\
$E_{V}$ & - & $(\mathrm{V} / \mathrm{m})^{2} / \mathrm{Hz}$ & $(\mathrm{V} / \mathrm{m})^{2} / \mathrm{Hz}$ & - & - & - \\
$E_{W}$ & - & - & $(\mathrm{V} / \mathrm{m})^{2} / \mathrm{Hz}$ & - & - & - \\
$B_{U}$ & $(\mathrm{nT} \mathrm{V} / \mathrm{m}) / \mathrm{Hz}$ & $(\mathrm{nT} \mathrm{V} / \mathrm{m}) / \mathrm{Hz}$ & $(\mathrm{nT} \mathrm{V} / \mathrm{m}) / \mathrm{Hz}$ & $\mathrm{nT}^{2} / \mathrm{Hz}$ & $\mathrm{nT}^{2} / \mathrm{Hz}$ & $\mathrm{nT}^{2} / \mathrm{Hz}$ \\
$B_{V}$ & $(\mathrm{nT} \mathrm{V} / \mathrm{m}) / \mathrm{Hz}$ & $(\mathrm{nT} \mathrm{V} / \mathrm{m}) / \mathrm{Hz}$ & $(\mathrm{nT} \mathrm{V} / \mathrm{m}) / \mathrm{Hz}$ & - & $\mathrm{nT}^{2} / \mathrm{Hz}$ & $\mathrm{nT}^{2} / \mathrm{Hz}$ \\
$B_{W}$ & $(\mathrm{nT} \mathrm{V} / \mathrm{m}) / \mathrm{Hz}$ & $(\mathrm{nT} \mathrm{V} / \mathrm{m}) / \mathrm{Hz}$ & $(\mathrm{nT} \mathrm{V} / \mathrm{m}) / \mathrm{Hz}$ & - & - & $\mathrm{nT}^{2} / \mathrm{Hz}$
\end{tabular}

Cadence: $6 \mathrm{~s}\left(E_{U}, E_{V}, E_{W}, B_{U}, B_{V}, B_{W}\right)$

16384 samples at $35 \mathrm{kSamples}$ per second (16 bit digitization)

Bin averaged into 65 logarithmically spaced bins between $2 \mathrm{~Hz}$ to $12 \mathrm{kHz}$

\section{A.3.3 WFR-Waveform}

6 channels $\left(E_{U}, E_{V}, E_{W}, B_{U}, B_{V}, B_{V}\right)$

Cadence: $\approx$ once per 15 minutes

16384 samples at $35 \mathrm{kSamples}$ per second (16 bit digitization)

A.4 Waves Burst Mode Products (Commandable and Limited by Memory Allocations)

\section{A.4.1 HFR-Waveform}

Cadence: commandable (not a standard product)

4096 samples at 1 MSamples per second (14 bit digitization)

\section{A.4.2 HFR-Spectra-Burst}

$E_{U}$ or $E_{V}$ or $E_{W}$

Cadence: $0.5 \mathrm{~s}$

4096 samples at 1 MSamples per second (14 bit digitization)

Bin averaged into 82 logarithmically spaced bins between $10 \mathrm{kHz}$ to $500 \mathrm{kHz}$ 


\section{A.4.3 WFR-Spectral-Matrix-Burst}

Similar to the standard survey product described above.

Cadence: $1 \mathrm{~s}$

16384 samples at $35 \mathrm{kSamples}$ per second (16 bit digitization)

Bin averaged into 65 logarithmically spaced bins between $2.14 \mathrm{~Hz}$ to $11.2 \mathrm{kHz}$

\section{A.4.4 WFR-Spectral-Matrix-30 ms}

6 channels

Cadence: $0.5 \mathrm{~s}$

16 sets of 1024 samples at $35 \mathrm{kSamples}$ per second (16 bit digitization)

\section{A.4.5 WFR-Spectra-30 ms-Mode}

6 channels $\left(E_{U}, E_{V}, E_{W}, B_{U}, B_{V}, B_{V}\right)$

Cadence: $30 \mathrm{~ms}$

1024 samples at $35 \mathrm{kSamples}$ per second (16 bit digitization)

Bin averaged into 70 linearly spaced bins (170 Hz band width) between $102.5 \mathrm{~Hz}$ to $11.894 \mathrm{kHz}$

\section{A.4.6 Wave Normal Analysis Mode (WNA Mode)}

Onboard processing of wave normal parameters based on $30 \mathrm{~ms}$ mode spectral matrix data.

As a function of frequency:

- $B^{2}$-Squared magnetic field magnitude, $\mathrm{nT}^{2} / \mathrm{Hz}$

- $E^{2}$-Squared electric field magnitude, $(\mathrm{V} / \mathrm{m})^{2} / \mathrm{Hz}$

- $S_{x}$-Poynting Vector, $\mathrm{W} / \mathrm{m}^{2}$

- $S_{y}$-Poynting Vector, $\mathrm{W} / \mathrm{m}^{2}$

- $S_{z}$-Poynting Vector, $\mathrm{W} / \mathrm{m}^{2}$

- Magnetic eigenvectors and eigenvalues-arbitrary units with component values for vectors.

\section{A.4.7 WFR-Waveform-Burst}

6 channels $\left(E_{U}, E_{V}, E_{W}, B_{U}, B_{V}, B_{V}\right)$

16384 samples at $35 \mathrm{kSamples}$ per second (16 bit digitization) for $0.468 \mathrm{~s}$

\section{A.4.8 WFR-Waveform-Continuous-Burst}

6 channels $\left(E_{U}, E_{V}, E_{W}, B_{U}, B_{V}, B_{V}\right)$

208896 samples at $35 \mathrm{kSamples}$ per second (16 bit digitization) for $5.968 \mathrm{~s}$

\section{Appendix B: Acronym List}

1PPS One Pulse Per Second signal

A/D or ADC Analog to Digital Converter

AC Alternating Current 


\begin{tabular}{|c|c|}
\hline AHBROM & Advanced High speed Bus Read Only Memory \\
\hline APID & Application Identifier \\
\hline CCSDS & Consultative Committee for Space Data Systems \\
\hline $\mathrm{CDF}$ & Common Data Format \\
\hline CDPU & Central Data Processing Unity \\
\hline C-RAM & Chalcogenide Random Access Memory \\
\hline DSP & Digital Signal Processor \\
\hline $\mathrm{ECH}$ & Electron Cyclotron Harmonic \\
\hline EDAC & Error Detection and Correction \\
\hline EEPROM & Electrically Erasable Programmable Read Only Memory \\
\hline EFW & Electric Field and Waves Instrument \\
\hline ELF & Extremely Low Frequency \\
\hline EMFISIS & The Electric and Magnetic Field Instrument Suite and Integrated Science \\
\hline EMIC & Electromagnetic Ion Cyclotron \\
\hline FFT & Fast Fourier Transform \\
\hline FIFO & First In First Out \\
\hline FPGA & Field Programmable Gate Array \\
\hline FSM & Finite State Machine \\
\hline GEI & Geocentric Equatorial Inertial \\
\hline GEO & Geographic \\
\hline GSE & Ground Support Equipment \\
\hline GSE & Geocentric Solar Ecliptic \\
\hline GSFC & Goddard Space Flight Center \\
\hline GSM & Geocentric Solar Magnetic \\
\hline HFR & High Frequency Receiver \\
\hline HK & Housekeeping \\
\hline IDL & Interactive Data Language \\
\hline IEM & Instrument Electronics Module \\
\hline ITF & Instrument Transfer Frame \\
\hline LVPS & Low Voltage Power Supply \\
\hline MAG & Fluxgate Magnetometer \\
\hline MEB & Main Electronics Box \\
\hline MET & Mission Elapsed Time \\
\hline MHD & Magnetohydrodynamics \\
\hline MI & Magnetosphere-Ionosphere \\
\hline MLT & Magnetic Local Time \\
\hline MOC & Mission Operations Center \\
\hline MSC & Magnetic Search Coil \\
\hline NASA & National Aeronautics and Space Administration \\
\hline NMI & Non-Maskable Interrupt \\
\hline RAM & Random Access Memory \\
\hline RBSP & Radiation Belt Storm Probes \\
\hline SAPS & Sub-Auroral Polarization Stream \\
\hline SASOC & Science Analysis Science Operations Center \\
\hline SCB & Self-Consistent B \\
\hline SDRAM & Synchronous Dynamic Random Access Memory \\
\hline SEU & Single Event Upset \\
\hline SM & Solar Magnetic \\
\hline SRAM & Static Random Access Memory \\
\hline
\end{tabular}




$\begin{array}{ll}\text { TDAS } & \text { THEMIS Data Analysis System } \\ \text { THEMIS } & \text { Time History of Events and Mesoscale Interactions during Substorms mission } \\ \text { TLA } & \text { Three Letter Acronym } \\ \text { UI } & \text { The University of Iowa } \\ \text { ULF } & \text { Ultra Low Frequency } \\ \text { UNH } & \text { University of New Hampshire } \\ \text { UVW } & \text { Spacecraft Science Coordinate System } \\ \text { VHDL } & \text { Very high speed integrated circuit (VHSIC) Hardware Definition Language } \\ \text { VLF } & \text { Very Low Frequency } \\ \text { WFR } & \text { Waveform Receiver }\end{array}$

\section{References}

B. Abel, R.M. Thorne, Electron scattering loss in Earth's inner magnetosphere. 1. Dominant physical processes. J. Geophys. Res. 103(A2) (1998a)

B. Abel, R.M. Thorne, Electron scattering loss in Earth's inner magnetosphere. 2. Sensitivity to model parameters. J. Geophys. Res. 103(A2) (1998b)

M.H. Acuna, Fluxgate magnetometers for outer planets exploration. IEEE Trans. Magn. 10 (1974)

M.H. Acuna, Space-based magnetometers. Rev. Sci. Instrum. 73 (2002)

J.M. Albert, Nonlinear interaction of outer zone electrons with VLF waves. Geophys. Res. Lett. 29(8) (2002)

J.M. Albert, Evaluation of quasi-linear diffusion coefficients for emic waves in a multispecies plasma. J. Geophys. Res. 108(A6) (2003)

J.M. Albert, N.P. Meredith, R.B. Horne, Three-dimensional diffusion simulation of outer radiation belt electrons during the 9 October 1990 magnetic storm. J. Geophys. Res. 114 (2009)

R.L. Arnoldy, M.J. Engebretson, R.E. Denton, J.L. Posch, M.R. Lessard, N.C. Maynard, D.M. Ober, C.J. Farrugia, C.T. Russell, J.D. Scudder, R.B. Torbert, S.-H. Chen, T.E. Moore, Pc1 waves and associated unstable distributions of magnetospheric protons observed during a solar wind pressure pulse. J. Geophys. Res. 110 (2005)

J.B. Blake, W.A. Kolasinski, R.W. Fillius, E.G. Mullen, Injection of electrons and protons with energies of tens of MeV into $L<3$ on 24 March 1991. Geophys. Res. Lett. 19 (1992)

J. Bortnik, R.M. Thorne, The dual role of ELF/VLF chorus waves in the acceleration and precipitation of radiation belt electrons. J. Atmos. Sol. Terr. Phys. 69 (2007)

J. Bortnik, R.M. Thorne, Transit time scattering of energetic electrons due to equatorially confined magnetosonic waves. J. Geophys. Res. 115 (2010)

J. Bortnik, R.M. Thorne, N.P. Meredith, Modeling the propagation characteristics of chorus using CRRES suprathermal electron fluxes. J. Geophys. Res. 112 (2007)

J. Bortnik, R.M. Thorne, U.S. Inan, Nonlinear interaction of energetic electrons with large amplitude chorus. Geophys. Res. Lett. 35 (2008a)

J. Bortnik, R.M. Thorne, N.P. Meredith, The unexpected origin of plasmaspheric hiss from discrete chorus emissions. Nature 452 (2008b)

J. Bortnik, R.M. Thorne, N.P. Meredith, Plasmaspheric hiss overview and relation to chorus. J. Atmos. Sol. Terr. Phys. 71 (2009a)

J. Bortnik, W. Li, R.M. Thorne, V. Angelopoulos, C. Cully, J. Bonnell, O.L. Contel, A. Roux, An observation linking the origin of plasmaspheric hiss to discrete chorus emissions. Science 324 (2009b)

D.H. Brautigam, G.P. Ginet, J.M. Albert, J.R. Wygant, D.E. Rowland, A. Ling, J. Bass, CRRES electric field power spectra and radial diffusion coefficients. J. Geophys. Res. 110 (2005)

A.W. Breneman, C.A. Kletzing, J. Pickett, J. Chum, O. Santolik, Statistics of multispacecraft observations of chorus dispersion and source location. J. Geophys. Res. 114 (2009)

L. Cahill Jr., Inflation of the inner magnetosphere during a magnetic storm. J. Geophys. Res. 71(19) (1966)

C. Cattell, J.R. Wygant, K. Goetz, K. Kersten, P.J. Kellogg, T. von Rosenvinge, S.D. Bale, I. Roth, M. Temerin, M.K. Hudson, R.A. Mewaldt, M. Wiedenbeck, M. Maksimovic, R. Ergun, M. Acuna, C.T. Russell, Discovery of very large amplitude whistler-mode waves in Earth's radiation belts. Geophys. Res. Lett. 35 (2008)

M.W. Chen, L.R. Lyons, M. Schulz, Simulations of phase space distributions of storm time proton ring current. J. Geophys. Res. 99(A4) (1994)

M.W. Chen, S. Liu, M. Schulz, J.L. Roeder, L.R. Lyons, Magnetically self-consistent ring current simulations during the 19 October 1998 storm. J. Geophys. Res. 111 (2006a) 
Y. Chen, R.H.W. Friedel, G.D. Reeves, Phase space density distribution of energetic electrons in the outer radiation belt during two geospace environment modeling inner magnetosphere/storms selected storms. J. Geophys. Res. 111 (2006b)

Y. Chen, G.D. Reeves, R.H.W. Friedel, The energization of relativistic electrons in the outer Van Allen radiation belt. Nat. Phys. 3 (2007)

L. Chen, R.M. Thorne, R.H. Horne, Simulation of emic excitation in a model magnetosphere including structured high-density plumes. J. Geophys. Res. 114 (2009a)

L. Chen, J. Bortnik, R.M. Thorne, R.B. Horne, V.K. Jordanova, Three-dimensional ray tracing of VLF waves in an asymmetric magnetospheric environment containing a plasmaspheric plume. Geophys. Res. Lett. $36(2009 b)$

L. Chen, R.M. Thorne, V.K. Jordanova, C.-P. Wang, M. Gkioulidou, L. Lyons, R.B. Horne, Global simulation of emic wave excitation during the 2001 April 21st storm from coupled RCM-RAM-hotray modeling. J. Geophys. Res. 115 (2010a)

L. Chen, R.M. Thorne, V.K. Jordanova, R.B. Horne, Global simulation of magnetosonic wave instability in the storm time magnetosphere. J. Geophys. Res. 115 (2010b)

F. Chu, M.K. Hudson, P. Haines, Y. Shprits, Dynamic modeling of radiation belt electrons by radial diffusion simulation for a 2 month interval following the 24 March 1991 storm injection. J. Geophys. Res. 115 (2010)

J. Chum, O. Santolik, A.W. Breneman, C.A. Kletzing, D.A. Gurnett, J.S. Pickett, Chorus source properties that produce time shifts and frequency range differences observed on different Cluster spacecraft. J. Geophys. Res. 112 (2007)

S.G. Claudepierre, S.R. Elkington, M. Wiltberger, Solar wind driving of magnetospheric ULF waves: pulsations driven by velocity shear at the magnetopause. J. Geophys. Res. 113 (2008)

S.G. Claudepierre, M. Wiltberger, S.R. Elkington, W. Lotko, M.K. Hudson, Magnetospheric cavity modes driven by solar wind dynamic pressure fluctuations. Geophys. Res. Lett. 36 (2009)

N. Cornilleau-Wehrlin, G. Chanteur, S. Perraut, L. Rezeau, P. Robert, A. Roux, C. de Villedary, P. Canul, M. Maksimovic, Y. de Conchy, D. Hubert, C. Lacombe, F. Lefeuvre, M. Parrot, J. Pincon, P. Decreau, C. Harvey, P. Louarn, O. Santolik, H. Alleyne, M. Roth, T. Chust, O. Le Contel, S. Team, First results obtained by the Cluster STAFF experiment. Ann. Geophys. 21(2), 437-456 (2003)

J.M. Cornwall, F.V. Coroniti, R.M. Thorne, Turbulent loss of ring current protons. J. Geophys. Res. 75 (1970)

M. Ejiri, Trajectory traces of charged particles in the magnetosphere. J. Geophys. Res. 83 (1978)

S.R. Elkington, M.K. Hudson, A.A. Chan, Acceleration of relativistic electrons via drift-resonant interaction with toroidal-mode PC-5 oscillations. Geophys. Res. Lett. 26 (1999)

S.R. Elkington, M.K. Hudson, A.A. Chan, Resonant acceleration and diffusion of outer zone electrons in an asymmetric geomagnetic field. J. Geophys. Res. 108(A3) (2003)

C.-G. Fälthammar, M. Walt, Radial motion resulting from pitch angle scattering of trapped electrons in the distorted geomagnetic field. J. Geophys. Res. 74 (1969)

Y. Fei, A.A. Chan, S.R. Elkington, M.J. Wiltberger, Radial diffusion and MHD particle simulations of relativistic electron transport by ULF waves in the September 1998 storm. J. Geophys. Res. 111 (2006)

M.-C. Fok, T.E. Moore, D.C. Delcourt, Modeling of inner plasma sheet and ring current during substorms. J. Geophys. Res. 104(A7) (1999)

M. Fok, R.B. Horne, N.P. Meredith, S.A. Glauert, Radiation belt environmental model: application to space weather nowcasting. J. Geophys. Res. 113 (2008)

J.C. Foster, H.B. Vo, Average characteristics and activity dependence of the subauroral polarization stream. J. Geophys. Res. 107(A12) (2002)

B.J. Fraser, R.S. Grew, S.K. Morley, J.C. Green, H.J. Singer, T.M. Loto'aniu, M.F. Thomsen, Stormtime observations of electromagnetic ion cyclotron waves at geosynchronous orbit: GOES results. J. Geophys. Res. 115 (2010)

N. Furuya, Y. Omura, D. Summers, Relativistic turning acceleration of radiation belt electrons by whistler mode chorus. J. Geophys. Res. 113 (2008)

N.Y. Ganushkina, T.I. Pulkkinen, V.A. Sergeev, M.V. Kubyshkina, D.N. Baker, N.E. Turner, M. Grande, B. Kellett, J. Fennell, J. Roeder, J.-A. Sauvaud, T.A. Fritz, Entry of plasma sheet particles into the inner magnetosphere as observed by Polar/Cammice. J. Geophys. Res. 105(A11) (2000)

J.C. Green, M.G. Kivelson, A tale of two theories: How the adiabatic response and ULF waves affect relativistic electrons. J. Geophys. Res. 106(A11) (2001)

J.C. Green, M.G. Kivelson, Relativistic electrons in the outer radiation belt: differentiating between acceleration mechanisms. J. Geophys. Res. 109 (2004)

N.M. Haque, M. Spasojevic, O. Santolik, U.S. Inan, Wave normal angles of magnetospheric chorus emissions observed on the Polar spacecraft. J. Geophys. Res. 115 (2010)

M. Hayosh, O. Santolik, M. Parrot, Location and size of the global source region of whistler mode chorus. J. Geophys. Res. 115 (2010) 
R.B. Horne, R.M. Thorne, Potential waves for relativistic electron scattering and stochastic acceleration during magnetic storms. Geophys. Res. Lett. 25 (1998)

R.B. Horne, R.M. Thorne, Electron pitch angle diffusion by electrostatic electron cyclotron waves: the origin of pancake distributions. J. Geophys. Res. 105(A3) (2000)

R.B. Horne, R.M. Thorne, Relativistic electron acceleration and precipitation during resonant interactions with whistler-mode chorus. Geophys. Res. Lett. 30(10) (2003)

R.B. Horne, G.V. Wheeler, H.S.C.K. Alleyne, Proton and electron heating by radially propagating fast magnetosonic waves. J. Geophys. Res. 105 (2000)

R.B. Horne, R.M. Thorne, N.P. Meredith, R.R. Anderson, Diffuse auroral electron scattering by electron cyclotron harmonic and whistler mode waves during an isolated substorm. J. Geophys. Res. 108(A7) (2003)

R.B. Horne, R.M. Thorne, S.A. Glauert, J.M. Albert, N.P. Meredith, R.R. Anderson, Timescales for radiation belt electron acceleration by whistler mode chorus waves. J. Geophys. Res. 110 (2005)

R.B. Horne, R.M. Thorne, S.A. Glauert, N.P. Meredith, D. Pokhotelov, O. Santolik, Electron acceleration in the Van Allen belts by fast magnetosonic waves. Geophys. Res. Lett. 34 (2007)

Y. Hu, R.E. Denton, Two-dimensional hybrid code simulation of electromagnetic ion cyclotron waves in a dipole magnetic field. J. Geophys. Res. 114 (2009)

C.L. Huang, H.E. Spence, M.K. Hudson, S.R. Elkington, Modeling radiation belt radial diffusion in ULF wave fields: 2. Estimating rates of radial diffusion using combined MHD and particle codes. J. Geophys. Res. 115 (2010)

M.K. Hudson, S.R. Elkington, J.G. Lyon, V.A. Marchenko, I. Roth, M. Temerin, J.B. Blake, M.S. Gussenhoven, J.R. Wygant, Simulations of radiation belt formation during storm sudden commencements. J. Geophys. Res. 102(A7) (1997)

M.K. Hudson, S.R. Elkington, J.G. Lyon, M. Wiltberger, M. Lessard, Radiation belt electron acceleration by ULF wave drift resonance: simulation of 1997 and 1998 storms, in Space Weather, ed. by P. Song, H. Singer, G. Siscoe. Geophys. Monogr., vol. 125 (AGU, Washington, 2001)

V.K. Jordanova, The role of the Earth's ring current in radiation belt dynamics, in Dynamics of the Earth's Radiation Belts and Inner Magnetosphere, vol. 199, ed. by D. Summers, I. Mann, D. Baker, M. Schulz (2012)

V.K. Jordanova, Y. Miyoshi, Relativistic model of ring current and radiation belt ions and electrons: initial results. Geophys. Res. Lett. 32 (2005)

V.K. Jordanova, J.U. Kozyra, A.F. Nagy, G.V. Khazanov, Kinetic model of the ring current-atmosphere interactions. J. Geophys. Res. 102 (1997)

V.K. Jordanova, L.M. Kistler, C.J. Farrugia, R.B. Torbert, Effects of inner magnetospheric convection on ring current dynamics: March 10-12, 1998. J. Geophys. Res. 106 (2001a)

V.K. Jordanova, C.J. Farrugia, R.M. Thorne, G.V. Khazanov, G.D. Reeves, M.F. Thomsen, Modeling ring current proton precipitation by electromagnetic ion cyclotron waves during the May 14-16, 1997 storm. J. Geophys. Res. 106 (2001b)

V.K. Jordanova, A. Boonsiriseth, R.M. Thorne, Y. Dotan, Ring current asymmetry from global simulations using a high-resolution electric field model. J. Geophys. Res. 108(A12) (2003)

V.K. Jordanova, Y.S. Miyoshi, S. Zaharia, M.F. Thomsen, G.D. Reeves, D.S. Evans, C.G. Mouikis, J.F. Fennell, Kinetic simulations of ring current evolution during the geospace environment modeling challenge events. J. Geophys. Res. 111 (2006)

V.K. Jordanova, M. Spasojevic, M. Thomsen, Modeling the electromagnetic ion cyclotron wave-induced formation of detached subauroral arcs. J. Geophys. Res. 112 (2007)

V.K. Jordanova, J. Albert, Y. Miyoshi, Relativistic electron precipitation by emic waves from self-consistent global simulations. J. Geophys. Res. 113 (2008)

V.K. Jordanova, R.M. Thorne, Y. Miyoshi, Excitation of whistler-mode chorus from global ring current simulations. J. Geophys. Res. 115 (2010a)

V.K. Jordanova, S. Zaharia, D.T. Welling, Comparative study of ring current development using empirical, dipolar, and self-consistent magnetic field simulations. J. Geophys. Res. 115 (2010b)

V.K. Jordanova, D.T. Welling, S.G. Zaharia, L. Chen, R.M. Thorne, Modeling ring current ion and electron dynamics and plasma instabilities during a high-speed stream driven storm. J. Geophys. Res. 117 (2012)

C.F. Kennel, R.M. Thorne, Unstable growth of unducted whistlers propagating at an angle to the geomagnetic field. J. Geophys. Res. 72 (1967)

H.-J. Kim, A.A. Chan, Fully adiabatic changes in storm time relativistic electron fluxes. J. Geophys. Res. 102 (1997)

B.T. Kress, M.K. Hudson, M.D. Looper, J. Albert, J.G. Lyon, C.C. Goodrich, Global MHD test particle simulations of $>10 \mathrm{MeV}$ radiation belt electrons during storm sudden commencement. J. Geophys. Res. 112 (2007) 
X. Li, I. Roth, M. Temerin, J.R. Wygant, M.K. Hudson, J.B. Blake, Simulation of the prompt energization and transport of radiation belt particles during the March 24, 1991 SSC. Geophys. Res. Lett. 20(22) (1993)

W. Li, R.M. Thorne, N.P. Meredith, R.B. Horne, J. Bortnik, Y.Y. Shprits, B. Ni, Evaluation of whistler mode chorus amplification during an injection event observed on CRRES. J. Geophys. Res. 113 (2008)

W. Li, R.M. Thorne, V. Angelopoulos, J.W. Bonnell, J.P. McFadden, C.W. Carlson, O. LeContel, A. Roux, K.H. Glassmeier, H.U. Auster, Evaluation of whistler-mode chorus intensification on the nightside during an injection event observed on the THEMIS spacecraft. J. Geophys. Res. 114 (2009a)

W. Li, R.M. Thorne, V. Angelopoulos, J. Bortnik, C.M. Cully, B. Ni, O. LeContel, A. Roux, U. Auster, W. Magnes, Global distribution of whistler-mode chorus observed on the THEMIS spacecraft. Geophys. Res. Lett. 36 (2009b)

M.W. Liemohn, J.U. Kozyra, M.F. Thomsen, J.L. Roeder, G. Lu, J.E. Borovsky, T.E. Cayton, Dominant role of the asymmetric ring current in producing the stormtime Dst*. J. Geophys. Res. 106(A6) (2001)

W. Liu, T.E. Sarris, X. Li, S.R. Elkington, R. Ergun, V. Angelopoulos, J. Bonnell, K.H. Glassmeier, Electric and magnetic field observations of Pc4 and Pc5 pulsations in the inner magnetosphere: a statistical study. J. Geophys. Res. 114 (2009)

T.M. Loto'aniu, B.J. Fraser, C.L. Waters, Propagation of electromagnetic ion cyclotron waves in the magnetosphere. J. Geophys. Res. 110 (2005)

T.M. Loto'aniu, I.R. Mann, L.G. Ozeke, A.A. Chan, Z.C. Dent, D.K. Milling, Radial diffusion of relativistic electrons into the radiation belt slot region during the 2003 Halloween storm. J. Geophys. Res. 111 (2006)

T.M. Loto'aniu, H.J. Singer, C.L. Waters, V. Angelopoulos, I.R. Mann, S.R. Elkington, J.W. Bonnell, Relativistic electron loss due to ultralow frequency waves and enhanced outward radial diffusion. J. Geophys. Res. 115 (2010)

L.R. Lyons, R.M. Thorne, Equilibrium structure of radiation belt electrons. J. Geophys. Res. 78 (1973)

L.R. Lyons, D.J. Williams, A source for the geomagnetic storm main phase ring current. J. Geophys. Res. 85(A2) (1980)

L.R. Lyons, R.M. Thorne, C.F. Kennel, Pitch angle diffusion of radiation belt electrons within the plasmasphere. J. Geophys. Res. 77 (1972)

R.A. Mathie, I.R. Mann, A correlation between extended intervals of ULF wave power and storm-time geosynchronous relativistic electron flux enhancements. Geophys. Res. Lett. 27 (2000)

J.P. McCollough, S.R. Elkington, D.N. Baker, Modelling emic wave growth during the compression event of 29 June 2007. Geophys. Res. Lett. 36 (2009)

N.P. Meredith, R.B. Horne, R.M. Thorne, R.R. Anderson, Favored regions for chorus-driven electron acceleration to relativistic energies in the Earth's outer radiation belt. Geophys. Res. Lett. 30(16) (2003a)

N.P. Meredith, R.M. Thorne, R.B. Horne, D. Summers, B.J. Fraser, R.R. Anderson, Statistical analysis of relativistic electron energies for cyclotron resonance with EMIC waves observed on CRRES. J. Geophys. Res. 108(A6) (2003b)

N.P. Meredith, R.B. Horne, R.R. Anderson, Survey of magnetosonic waves and proton ring distributions in Earth's inner magnetosphere. J. Geophys. Res. 113 (2008)

N.P. Meredith, R.B. Horne, R.M. Thorne, R.R. Anderson, Survey of upper band chorus and ech waves: implications for the diffuse aurora. J. Geophys. Res. 114 (2009)

R.M. Millan, R.M. Thorne, Review of radiation belt relativistic electron loss. J. Atmos. Sol. Terr. Phys. 69 (2007)

E.V. Mishin, W.J. Burke, C.Y. Huang, F.J. Rich, Electromagnetic wave structures within subauroral polarization streams. J. Geophys. Res. 108(A8) (2003)

Y. Miyoshi, V.K. Jordanova, A. Morioka, M.F. Thomsen, G.D. Reeves, D.S. Evans, J.C. Green, Observations and modeling of energetic electron dynamics during the October 2001 storm. J. Geophys. Res. 111 (2001)

Y. Miyoshi, V.K. Jordanova, M.F. Thomsen, G.D. Reeves, D.S. Evans, A. Morioka, Y. Kasahara, T. Nagai, J. Green, Simulation of energetic electrons dynamics on the Oct. 2001 magnetic storm. EOS Trans. AGU 84 (2003)

S.K. Morley, S.T. Ables, M.D. Sciffer, B.J. Fraser, Multipoint observations of Pc1-2 waves in the afternoon sector. J. Geophys. Res. 114 (2009)

S.K. Morley, R.H.W. Friedel, T.E. Cayton, E. Noveroske, A rapid, global and prolonged electron radiation belt dropout observed with the global positioning system constellation. Geophys. Res. Lett. 37 (2010)

N.F. Ness, Magnetometers for space research. Space Sci. Rev. 11 (1970)

B. Ni, R.M. Thorne, Y.Y. Shprits, J. Bortnik, Resonant scattering of plasma sheet electrons by whistler-mode chorus: contributions to diffuse auroral precipitation. Geophys. Res. Lett. 35 (2008)

B. Ni, R.M. Thorne, J. Liang, V. Angelopoulos, C. Cully, W. Li, X. Zhang, M. Hartinger, O.L. Contel, A. Roux, Global distribution of electrostatic electron cyclotron harmonic waves observed on THEMIS. Geophys. Res. Lett. 38 (2011) 
Y. Nishimura, J. Bortnik, W. Li, R.M. Thorne, L.R. Lyons, V. Angelopoulos, S. Mende, J.W. Bonnel, O. LeContel, U. Auster, Identifying the driver of pulsating aurora. Science 330 (2010)

T.P. O'Brien, R.L. McPherron, D. Sornette, G.D. Reeves, R. Friedel, H.J. Singer, Which magnetic storms produce relativistic electrons at geosynchronous orbit? J. Geophys. Res. 106 (2001)

N. Omidi, R.M. Thorne, J. Bortnik, Non-linear evolution of emic waves in a uniform magnetic field: 1 . Hybrid simulations. J. Geophys. Res. 115 (2010)

T.G. Onsager, J.C. Green, G.D. Reeves, H.J. Singer, Solar wind and magnetospheric conditions leading to the abrupt loss of outer radiation belt electrons. Geophys. Res. Lett. 112 (2007)

K.L. Perry, M.K. Hudson, S.R. Elkington, Incorporating spectral characteristics of Pc5 waves into threedimensional modeling and the diffusion of relativistic electrons. J. Geophys. Res. 110 (2005)

J.S. Pickett, B. Grison, Y. Omura, M.J. Engebretson, I. Dandouras, A. Masson, M.L. Adrian, O. Santolik, P.M.E. Decreau, N. Cornilleau-Wehrlin, D. Constantinescu, Cluster observations of EMIC triggered emissions in association with Pc1 waves near Earth's plasmapause. Geophys. Res. Lett. 37 (2010)

K.G. Powell, P.L. Roe, T.J. Linde, T.I. Gombosi, D.L. de Zeeuw, A solution-adaptive upwind scheme for ideal magnetohydrodynamics. J. Comput. Phys. 153 (1999)

C.E. Rasmussen, S.M. Guiter, S.G. Thomas, Two-dimensional model of the plasmasphere: refilling time constants. Planet. Space Sci. 41 (1993)

A.J. Ridley, M.W. Liemohn, A model-derived storm time asymmetric ring current driven electric field description. J. Geophys. Res. 107(A8) (2002)

J.G. Roederer, Dynamics of Geomagnetically Trapped Radiation (Springer, New York, 1970)

G. Rostoker, S. Skone, D.N. Baker, On the origin of relativistic electrons in the magnetosphere associated with some geomagnetic storms. Geophys. Res. Lett. 25 (1998)

O. Santolik, F. Lefeuvre, M. Parrot, J. Rauch, Complete wave-vector directions of electromagnetic emissions: application to INTERBALL-2 measurements in the nightside auroral zone. J. Geophys. Res. 106(A7), 13191-13201 (2001)

O. Santolik, M. Parrot, F. Lefeuvre, Singular value decomposition methods for wave propagation analysis. Radio Sci. 38(1) (2003)

O. Santolik, D.S. Gurnett, J.S. Pickett, M. Parrot, N. Cornilleau-Wehrlin, A microscopic and nanoscopic view of storm-time chorus on 31 March 2001. Geophys. Res. Lett. 31 (2004)

O. Santolik, D.A. Gurnett, J.S. Pickett, J. Chum, N. Cornilleau-Wehrlin, Oblique propagation of whistler mode waves in the chorus source region. J. Geophys. Res. 114 (2009)

M. Schulz, L. Lanzerotti, Particle Diffusion in the Radiation Belts (Springer, New York, 1974)

Y.Y. Shprits, B. Ni, Dependence of the quasi-linear scattering rates on the wave normal distribution of chorus waves. J. Geophys. Res. 114 (2009)

Y. Shprits, R.M. Thorne, Time dependent radial diffusion modeling of relativistic electrons with realistic loss rates. Geophys. Res. Lett. 31 (2004)

Y.Y. Shprits, R.M. Thorne, R. Friedel, G.D. Reeves, J. Fennell, D.N. Baker, S.G. Kanekal, Outward radial diffusion driven by losses at magnetopause. J. Geophys. Res. 111 (2006)

Y.Y. Shprits, D. Subbotin, B. Ni, Evolution of electron fluxes in the outer radiation belt computed with the verb code. J. Geophys. Res. 114 (2009)

D.A. Subbotin, Y.Y. Shprits, Three dimensional modeling of the radiation belts using the versatile electron radiation belt (verb) code. Space Weather 7 (2009)

D. Summers, Y. Omura, Ultra-relativistic acceleration of electrons in planetary magnetospheres. Geophys. Res. Lett. 34 (2007)

D. Summers, R.M. Thorne, Relativistic electron pitch-angle scattering by electromagnetic ion cyclotron waves during geomagnetic storms. J. Geophys. Res. 108(A4) (2003)

D. Summers, R.M. Thorne, F. Xiao, Relativistic theory of wave-particle resonant diffusion with application to electron acceleration in the magnetosphere. J. Geophys. Res. 103 (1998)

X. Tao, A.A. Chan, J.M. Albert, J.A. Miller, Stochastic modeling of multidimensional diffusion in the radiation belts. J. Geophys. Res. 113 (2008)

X. Tao, J.M. Albert, A.A. Chan, Numerical modeling of multidimensional diffusion in the radiation belts using layer methods. J. Geophys. Res. 145 (2009)

R.M. Thorne, Radiation belt dynamics: the importance of wave-particle interactions. Geophys. Res. Lett. 37 (2010)

R.M. Thorne, R.B. Horne, Modulation of electromagnetic ion cyclotron instability due to interaction with ring current o+ during geomagnetic storms. J. Geophys. Res. 102(A7) (1997)

R.M. Thorne, C.F. Kennel, Relativistic electron precipitation during magnetic storm main phase. J. Geophys. Res. 76 (1971)

R.M. Thorne, T.P. O’Brien, Y.Y. Shprits, D. Summers, R.B. Horne, Timescale for MeV electron microburst loss during geomagnetic storms. J. Geophys. Res. 110 (2005) 
R.M. Thorne, X.T. B. Ni, R.B. Horne, N.P. Meredith, Scattering by chorus waves as the dominant cause of diffuse auroral precipitation. Nature 467 (2010)

B.T. Tsurutani, E.J. Smith, Postmidnight chorus: a substorm phenomenon. J. Geophys. Res. 79 (1974)

B.T. Tsurutani, E.J. Smith, Two types of magnetospheric elf chorus and their substorm dependences. J. Geophys. Res. 82 (1977)

B.T. Tsurutani, O.P. Verkhoglyadova, G.S. Lakhina, S. Yagitani, Properties of dayside outer zone chorus during HILDCAA events: loss of energetic electrons. J. Geophys. Res. 114 (2009)

N.A. Tsyganenko, D.P. Stern, Modeling the global magnetic field of the large-scale Birkeland current systems. J. Geophys. Res. 101 (1996)

N.A. Tsyganenko, H.J. Singer, J.C. Kasper, Storm-time distortion of the inner magnetosphere: how severe can it get? J. Geophys. Res. 108 (2003)

W. Tu et al., Storm-dependent radiation belt electron dynamics. J. Geophys. Res. 114 (2009)

A.Y. Ukhorskiy, K. Takahashi, B.J. Anderson, H. Korth, Impact of toroidal ULF waves on outer radiation belt electrons. J. Geophys. Res. 110 (2005)

A.Y. Ukhorskiy, B.J. Anderson, K. Takahashi, N.A. Tsyganenko, Impact of ULF oscillations in solar wind dynamic pressure on the outer radiation belt electrons. Geophys. Res. Lett. 33 (2006)

A.Y. Ukhorskiy, M.I. Sitnov, K. Takahasi, B.J. Anderson, Radial transport of radiation belt electrons due to stormtime Pc5 waves. Ann. Geophys. 27 (2009)

M.E. Usanova et al., Multipoint observations of magnetospheric compression-related emic Pc1 waves by THEMIS and Carisma. Geophys. Res. Lett. 35 (2008)

A.L. Vampola, A. Korth, Electron drift echoes in the inner magnetosphere. Geophys. Res. Lett. 19 (1992)

A. Varotsou, D. Boscher, S. Bourdarie, R.B. Horne, N.P. Meredith, S.A. Glauert, R.H. Friedel, Three dimensional test simulations of the outer radiation belt electron dynamics including electron-chorus resonant interactions. J. Geophys. Res. 113 (2008)

D.R. Weimer, An improved model of ionospheric electric potentials including substorm perturbations and application to the Geospace Environment Modeling November 24, 1996, event. J. Geophys. Res. 106 (2001)

R.A. Wolf, J.W. Freeman Jr., B.A. Hausman, R.W. Spiro, R.V. Hilmer, R.L. Lambour, Modeling Convection Effects in Magnetic Storms. Geophys. Monogr., vol. 98 (AGU, Washington, 1997)

J. Wygant, F. Mozer, M. Temerin, J. Blake, N. Maynard, H. Singer, M. Smiddy, Large amplitude electric and magnetic field signatures in the inner magnetosphere during injection of $15 \mathrm{MeV}$ electron drift echoes. Geophys. Res. Lett. 21 (1994)

Y. Yu, V. Jordanova, S. Zaharia, J. Koller, J. Zhang, L.M. Kistler, Validation study of the magnetically selfconsistent inner magnetosphere model RAM-SCB. J. Geophys. Res. 117 (2012)

S. Zaharia, V.K. Jordanova, M.F. Thomsen, G.D. Reeves, Self-consistent modeling of magnetic fields and plasmas in the inner magnetosphere: application to a geomagnetic storm. J. Geophys. Res. 111 (2006)

S. Zaharia, V.K. Jordanova, D.T. Welling, G. Toth, Self-consistent inner magnetosphere simulation driven by a global MHD model. J. Geophys. Res. 115 (2010) 